\title{
Low Cost Framework for Non-Intrusive Load Monitoring (NILM) to Monitor Human Behavioral Pattern
}

\author{
by \\ Omid Pezeshkfar \\ M.S. Electrical Engineering, University of Missouri-Columbia
}

Advisor: Dr. David Heise

Electrical and Computer Engineering Department

5 December 2013 
The undersigned, appointed by the dean of the Graduate School, have examined the thesis entitled

\section{Low Cost Framework for Non-Intrusive Load Monitoring (NILM) to Monitor Human Behavioral Pattern}

presented by Omid Pezeshkfar

a candidate for the degree of master of Electrical and Computer Engineering, and hereby certify that, in their opinion, it is worthy of acceptance.

Professor David Heise

Professor Michael J. Devaney

Professor William H. Miller 


\section{Acknowledgments}

Foremost, I would like to express my sincere gratitude to my advisor Prof. Heise for the continuous support of my Master's study and research, for his patience, motivation, enthusiasm, and immense knowledge. His guidance helped me in all the time of research and writing of this thesis. I could not have imagined having a better advisor and mentor for my study.

Besides my advisor, I would like to thank the rest of my thesis committee: Prof. Miller and Prof. Devaney for their encouragement, insightful comments, hard questions and providing this opportunity to complete my research.

Last but not the least, I would like to thank my family: my parents Mohammadreza Pezeshkfar \& Nahid Loghmani, for giving birth to me at the first place and supporting me spiritually throughout my life. 


\section{Table of Contents}

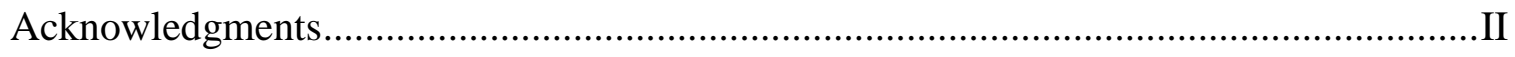

Table of Contents ............................................................................................. III

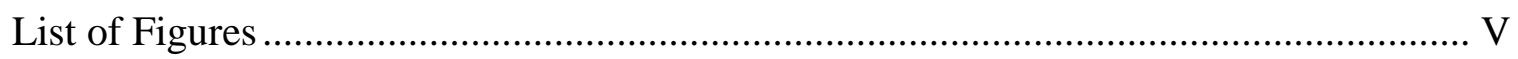

List of Tables ................................................................................................ VIII

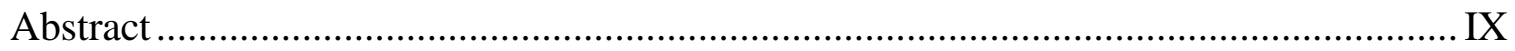

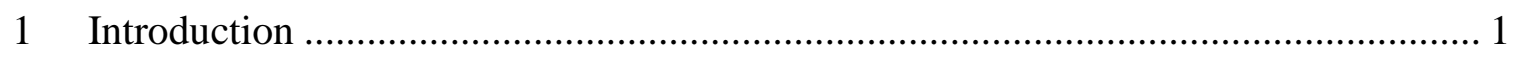

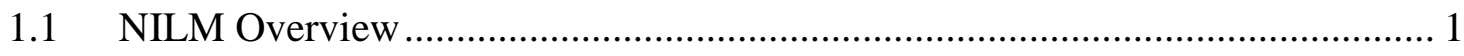

1.2 Motivation for Research.......................................................................... 3

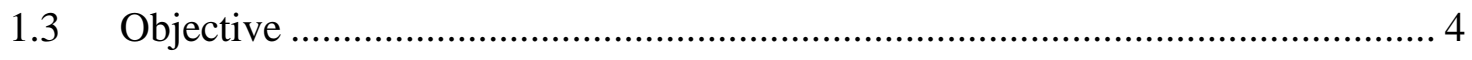

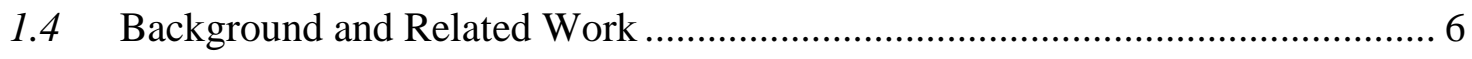

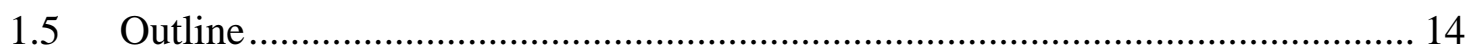

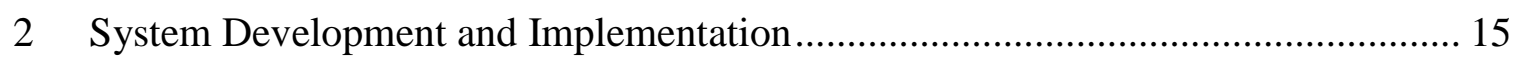

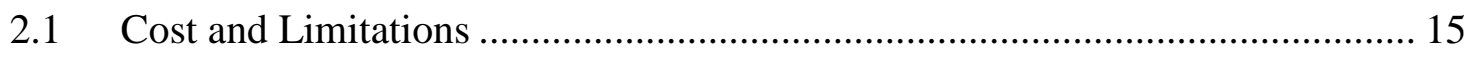

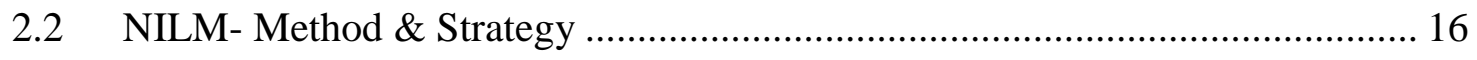

2.3 Developing Hardware Framework ........................................................ 18

2.3.1 NILM Hardware Framework- First Part ….................................................... 20

2.3.2 NILM Hardware Framework- Second Part.................................................... 29

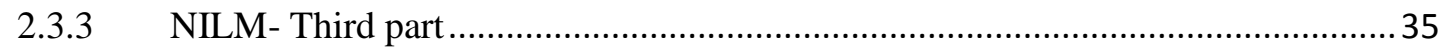

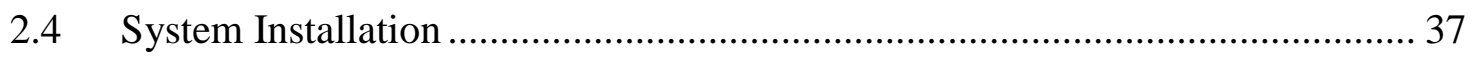




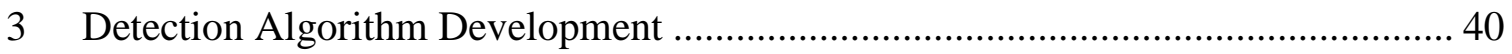

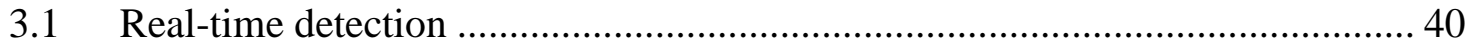

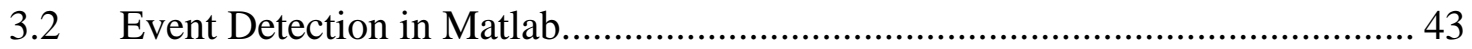

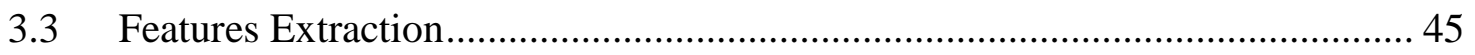

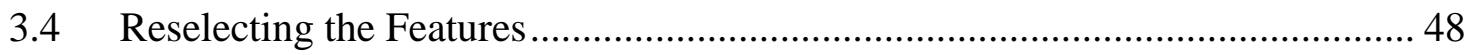

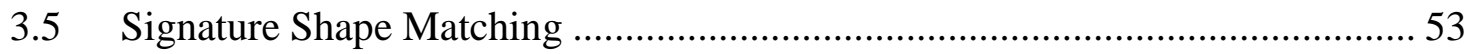

3.6 Automated Event Detection/Classification ................................................. 56

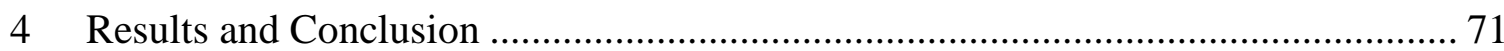

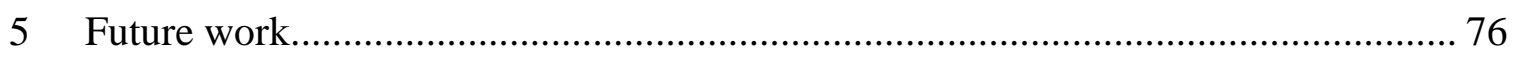

5.1 Current Systems ................................................................................... 76

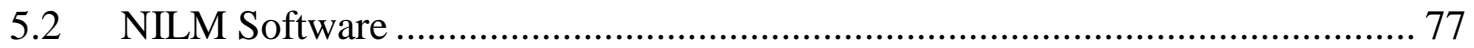

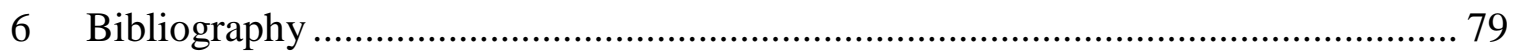




\section{List of Figures}

Figure 1. Block diagram representation of a NILM system. ...................................... 2

Figure 2. Block diagram of representative NILM process. ........................................ 5

Figure 3: Designed architecture for NILM [8]. .................................................... 7

Figure 4: Raw AC voltage and current measurement captured during motor start transient

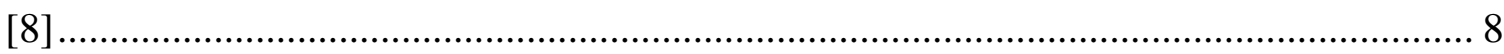

Figure5: Voltage and current signals shown in previous figure are converted into a power

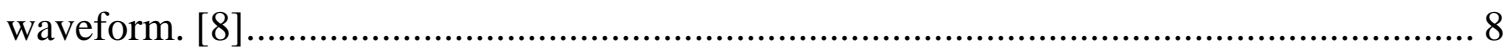

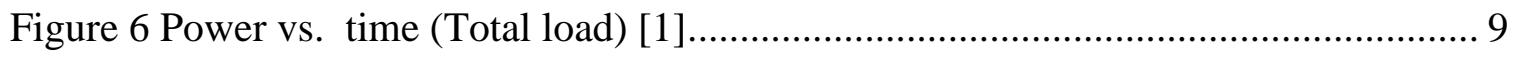

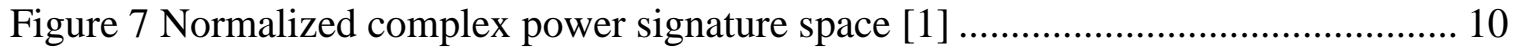

Figure 8 A graphical illustration of wave-shape metrics: (a) V-I trajectory. (b) Mean curve.

(c) Reference line joining points of highest and lowest I-coordinate in the V-I plane. From

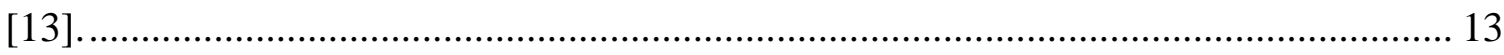

Figure 9 Eldercare House Modified NILM Architecture .......................................... 16

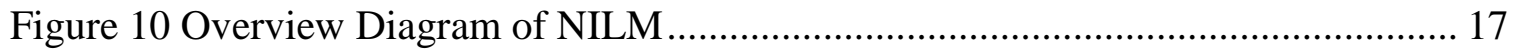

Figure 11 Data Acquisition \& Signal Preprocessing Part of NILM ............................. 19

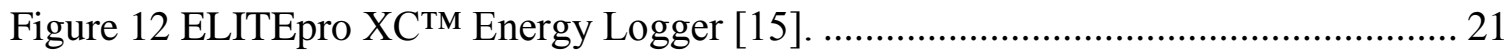

Figure 13 Left: Pico current clamp 1mV/A, Right :Mastech current clamp 10 mV/A .... 21

Figure 14 Current Clamps Installed inside the Circuit Breaker.................................. 22

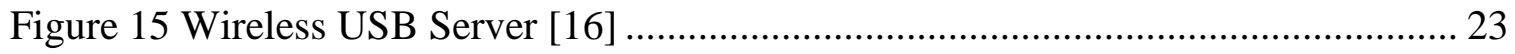

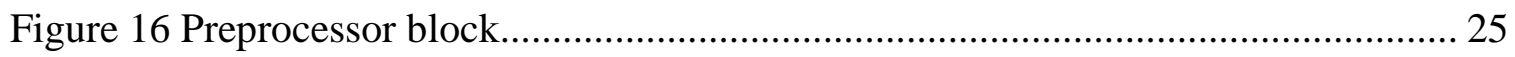

Figure 17. Voltage and current phase relationships for 3 components in PQ plane....... 26

Figure 18 Simulating voltage signals for 3 phase system based on one phase data ........ 29 
Figure 19 Oscilloscope Setup in LabView+ Main Signal calculation . 31

Figure 20 Power Suit module in LabView ................................................................... 33

Figure 21 Automatic File path maker- LabView Module .......................................... 34

Figure 22 Sliding average window on power signal detecting events in the real-time .... 36

Figure 23 Oscilloscope and wireless USB adaptor ............................................. 38

Figure 24 NILM wireless data Acquisition installed inside the circuit breaker .............. 39

Figure 25 Developed Module to detect the peaks and valleys with their values ............. 41

Figure 26 An Example of Real-time detection with developed module in LabView....... 42

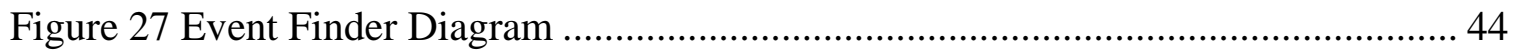

Figure 28 Start-up transient example of three different device .................................. 44

Figure 29 Features used to detect signals a. Overshoot, b. signal average, c. AUC (Area

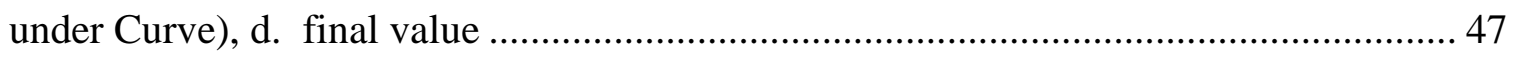

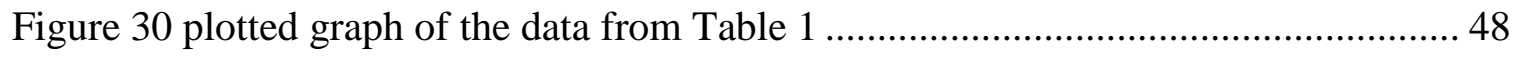

Figure 31 Overshoot time difference between the two same power signatures for the same

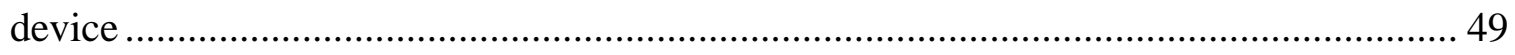

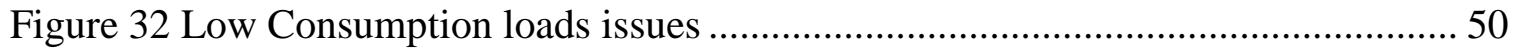

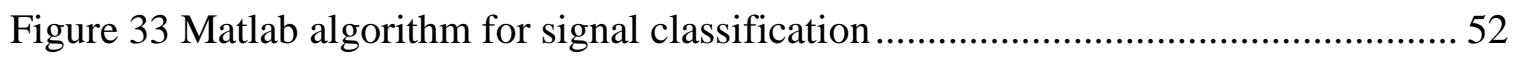

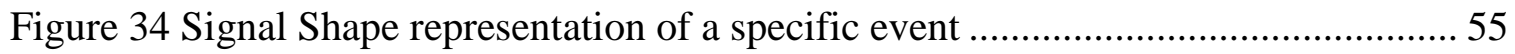

Figure 35 Two events with the same signature .................................................. 55

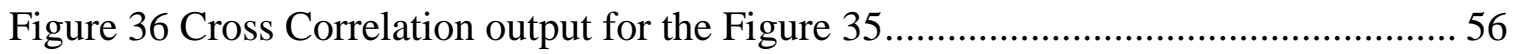

Figure 37 Representation of Active and Reactive Power Signals of Table above. .......... 57

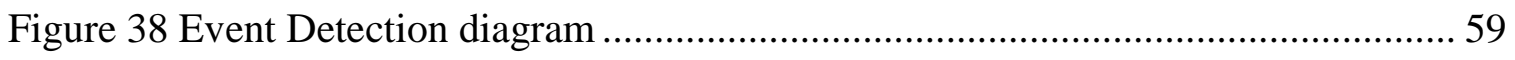


Figure 39 Turning ON-OFF power signal (up), cut and trimmed turning ON power signal signature (down-right) cut and trimmed turning OFF power signal signature (down-left)

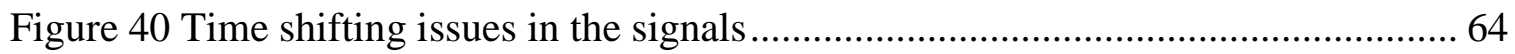

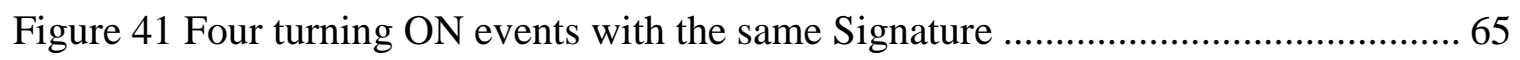

Figure 422 Features of Active Power for four turning ON events shown in Figure 42 ... 65

Figure 433 Four turning OFF events with the same Signature ................................... 66

Figure 44 Features of Reactive Power for four turning ON events shown in Figure 42 .. 66

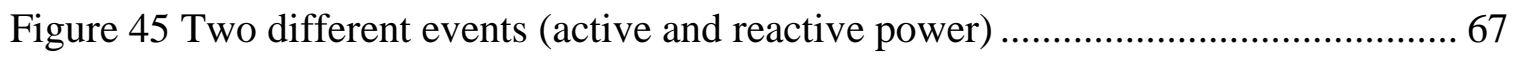

Figure 46 Comparing features of two events shown in Figure 45 .............................. 68

Figure 47 Visual Representation of the number of turning ON and OFF for each appliances

Figure 48 Visual Representation of the percentage of ONLY turning ON for each

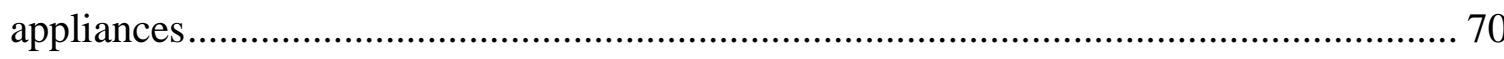

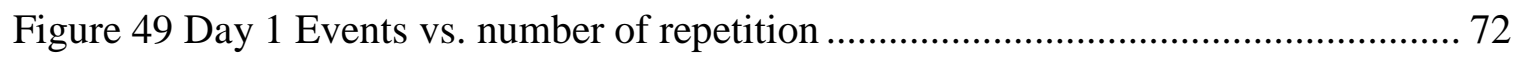

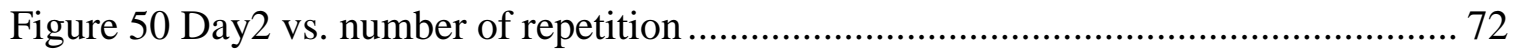

Figure 51 Appliances usage pattern for two days ................................................. 73

Figure 52 One day pattern for all events ( $\mathrm{V}$ axis: event number, $\mathrm{H}$ axis: time of day in

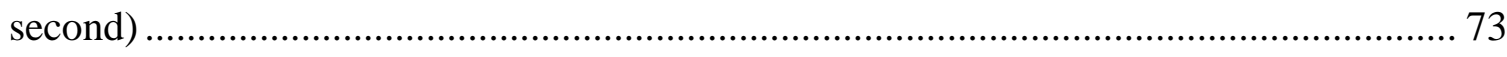

Figure 53 Left, Turn ON signature of a sample small load, Right: Turn OFF signatures of a sample small load 75 


\section{List of Tables}

Table 1 A sample of Featre Matrix with the signatre data......................................... 477

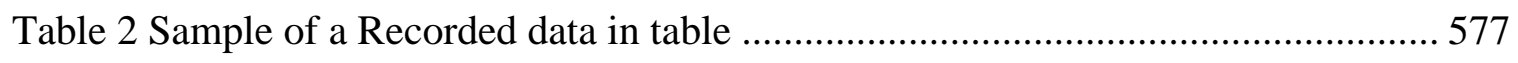




\title{
Low Cost Framework for Non-Intrusive Load Monitoring (NILM) to Monitor Human Behavioral Pattern \\ Omid Pezeshkfar \\ Dr. David Heise, Thesis Supervisor
}

\begin{abstract}
Non-Intrusive Load Monitoring (NILM) is a technique that measures and samples voltage and current from an electrical power source in order to infer what devices or appliances are being used at a given time. A NILM system can monitor a single phase or 3-phase electrical system. This system basically captures those waveforms and then calculates the power waveforms for each phase. Thus, a NILM system may monitor the electrical system from a single reference point, such as a circuit breaker box. The associated software is the biggest challenge in this area, as it has to detect on/off events for each device using only the power waveforms.
\end{abstract}

This thesis presents a low cost framework design and hardware implementation of NILM designed to monitor residential activities inside the home. The motivation for this work is specifically to monitor seniors in their homes, such as is currently done in residential living communities such as TigerPlace in Columbia, Missouri. The goal of this work is to be able to monitor the usage of appliances and electrical devices inside the home, thereby allowing analysis of the daily, monthly, or yearly pattern of usage that might reveal changes in an everyday routine. A key criterion for developing a monitoring system for the home is to protect the privacy of residents; NILM is a natural choice because it does not require any intrusive sensors.

The development, testing, and results from deploying a prototype NILM system are described, along with ideas for extending the work of this thesis. 


\section{Introduction}

\subsection{NILM Overview}

Non-intrusive load monitoring (NILM), or non-intrusive appliance load monitoring (NIALM), is a technique for analyzing changes in the voltage and current from a power source into a building (or other electrical subsystem) and deducing what appliances are used as well as their individual energy consumption. Put another way, the NonIntrusive Load Monitor (NILM) is a device that utilizes electrical voltage and current waveform to determine the operating schedule of major loads. NILM is considered a low cost alternative to attaching individual monitors on each appliance. It was initially developed in 1982 at MIT by George W. Hart. While looking at load data for a photovoltaic study, the research team noticed that on/off events for major appliances in the home could be read visually [1].

The non-intrusive aspect of the device is its minimal impact on an existing system. For instance, it could easily install on a meter without shutting the power. Simple wire connections are used to monitor the voltage, and a current transducer is used to measure the aggregate current. These raw measurements are analyzed by the installed software to calculate the real and reactive power which in turn can be used (for example) to perform diagnostics on the electrical system or record the activities of residents in their home. 
NILM can detect what types of appliances people have and their behavioral patterns. Patterns of energy use may indicate behavior patterns, such as routine times that nobody is at home, or unusual behavior of residents. It could, for example, reveal when the occupants of a house are using the shower, or when individual lights are turned on and off or even when the occupants of a house are watching TV or cooking and so much more. Figure 1 represents a possible configuration of a NILM system.

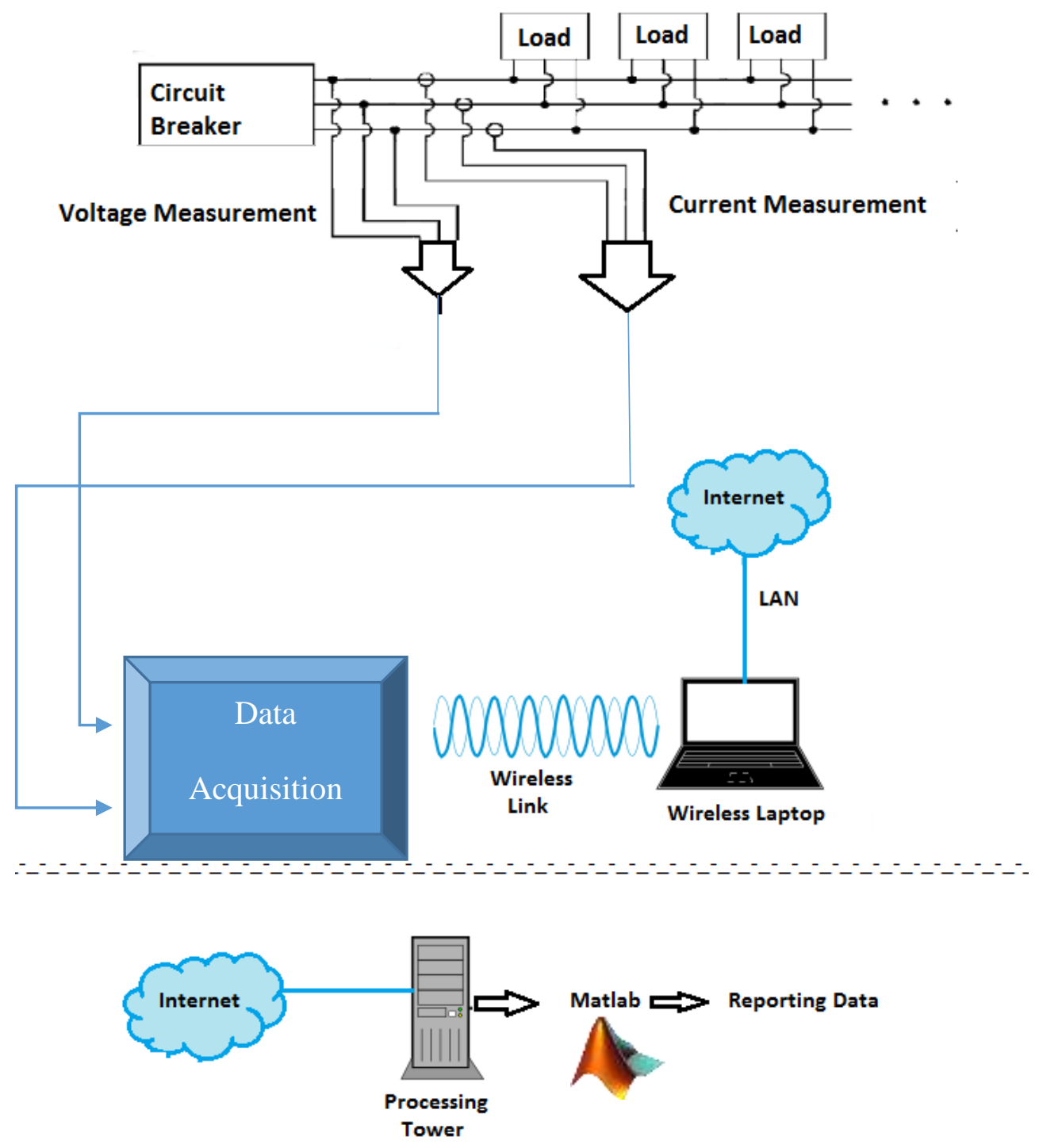

Figure 1. Block diagram representation of a NILM system. 


\subsection{Motivation for Research}

Chronic disease management is the biggest health care problem facing the United States today [2]. In 2005, almost 1 in 2 Americans (133 million) had at least one chronic condition or cognitive problems, and $21 \%$ of the population had multiple chronic conditions. These numbers are predicting to continually increase over the next 30 years. Chronic diseases especially affect older adults in whom exacerbations result in dramatic changes and decline in health status, hospitalization, complex treatment interventions, and high cost. Early illness recognition and early treatment is not only a key to improve health status with rapid recovery after an exacerbation of a chronic illness or acute illness, but also a key to reducing morbidity and mortality in older adults and controlling costs of health care [2].

NILM research has proved that power signature can provide useful and cost-efficient tool in recognizing the events in the system and also to diagnostic the system to find the unusual and abnormal activities. NILM can contribute in the of monitoring elders and develop alerts based on the data to notify health care providers of early signs of illness or functional decline so they could further evaluate and intervene with early treatment. A combination of NILM and some other behavioral controlling systems can show the clinical effectiveness and cost-effectiveness of using that in elder housing. The data that is collected with NILM can feed into automated algorithms for illness detection to find earlier signs of illness or functional decline in older adults compared to usual health assessment. Helping older adults remain healthier, active, and control their chronic illnesses with early detection of 
changes in health status and early intervention by health care providers, millions can remain independent as they age, avoiding or reducing debilitating and costly hospital stays, and for many, avoiding or delaying the move to a nursing home [2].

The proposed technologies will be tested and evaluated at TigerPlace, a unique independent-living, apartment-style eldercare facility designed by the Sinclair School of Nursing at University of Missouri-Columbia (MU). Access to this cutting edge facility affiliated with MU provides an exceptional opportunity to accomplish our research aims. The potential risks of the elderly people living alone and the dreadful consequences of late response to abnormal situations call for the proposed technology. The remarkable growth of the elderly population and the shortage of eldercare professionals underscore the significance of the proposed research.

\subsection{Objective}

The findings of the most recent NILM research has shown that the electrical system behavior was evident in the system's power signature and in many cases these data can be used to detect and analyze the system [3] [4] [5] [6]. Section 1.4 provides abstracts of some of the past research that are related to this thesis. As is already mentioned, we hope NILM can contribute to automated illness detection methods to find early signs of illness or functional decline in older adults. The objective of this research is to implement, improve and validate a low cost framework that can be used to allow detection and classification of 
on/off events for each appliance in the residential home. Furthermore, this data can be used for illness detection and further analysis.

In this thesis and project, the main goal is finding a low cost way to detect behavioral pattern of using appliances in a residential household. Since elders have regular routines for everyday activities and use almost the same devices and appliances every day, it would be valuable to implement a system to detect the usage of these electric devices and subsequently determine a pattern of behavior for each resident. Figure 2 demonstrate one possible framework for accomplishing this task.

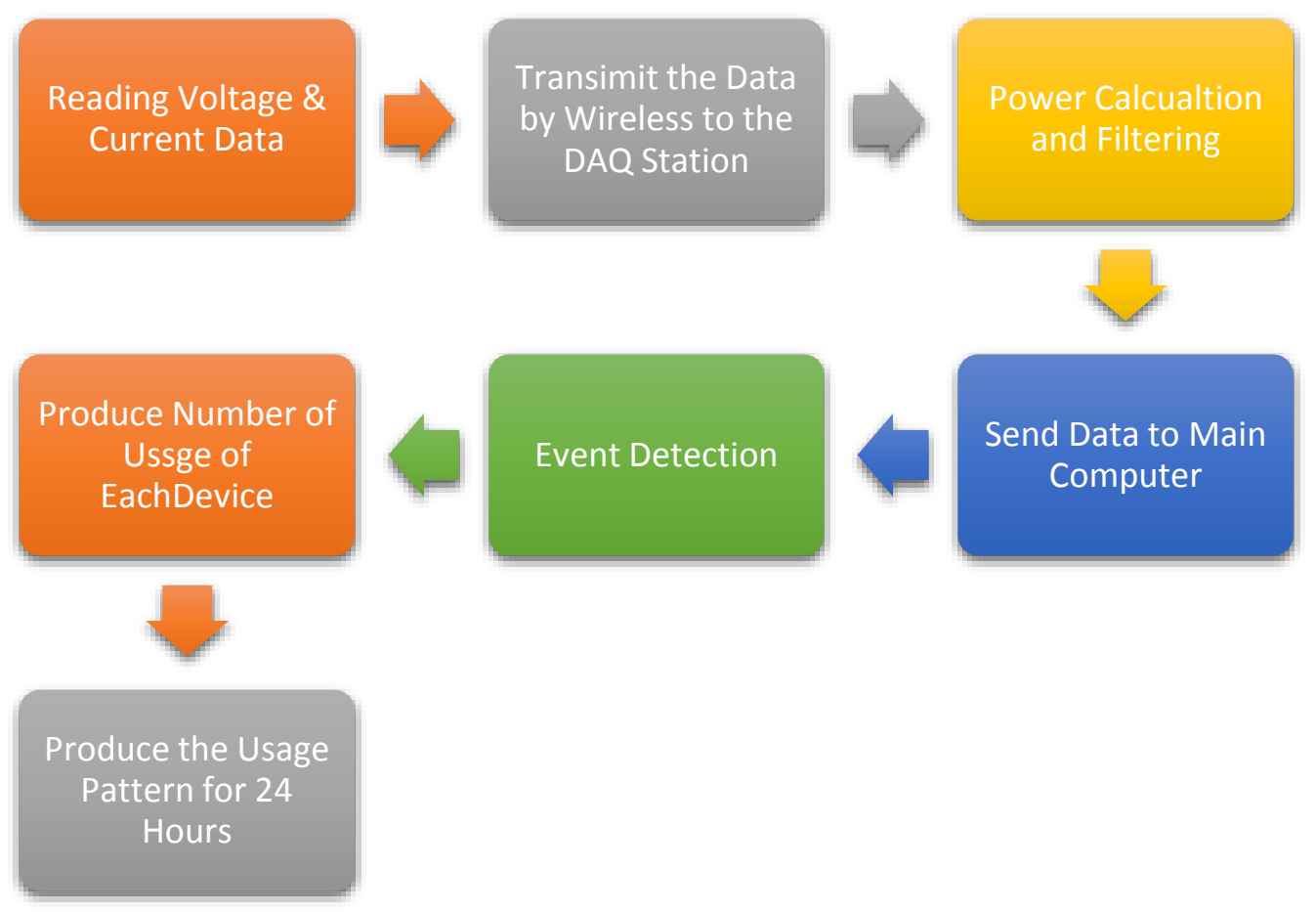

Figure 2. Block diagram of representative NILM process. 


\subsection{Background and Related Work}

Non-intrusive Load Monitoring was invented by George W. Hart, Ed Kern and Fred Schweppe of MIT in the early 1980s with funding from the Electric Power Research

Institute [7]. He discovered the NILM idea while he was looking at load data for a photovoltaic study, his research team found out that on/off events for major appliances can be read visually thought the power signals. Hart developed this concept into a system where he focused on the amplitude of the active and reactive power consumption of devices and classification in PQ plane. He also found the various states of different devices by using an extraction algorithm to find when devices turn on and off.

In Figure 3 you can see a designed architecture for NILM [1]. It captures signals of voltage and current at each phase and which should be preprocessed to make them ready for the next stage. Preprocessing might include filtering noise or adjusting the phase of each line. Note that NILM measures the aggregate current flowing for the composite load and then by using signal processing technique can disaggregate the operating status of individual loads. 


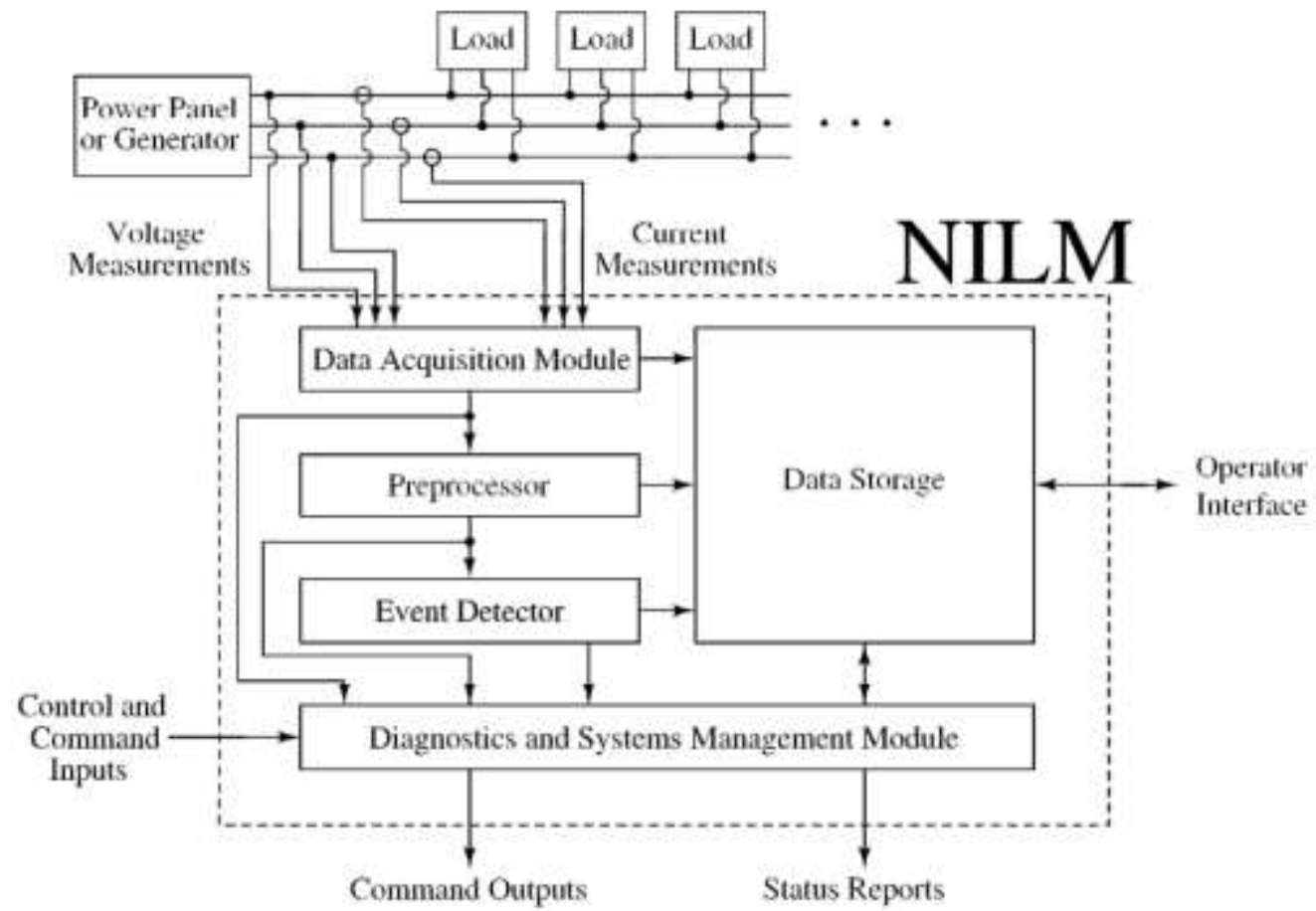

Figure 3: Designed architecture for NILM [8].

After finding the waveforms of voltage and current, the power signal is generated simply by multiplying point-by-point voltage waveforms to the current. In the Figure.4 the voltage and current waveforms are shown. 


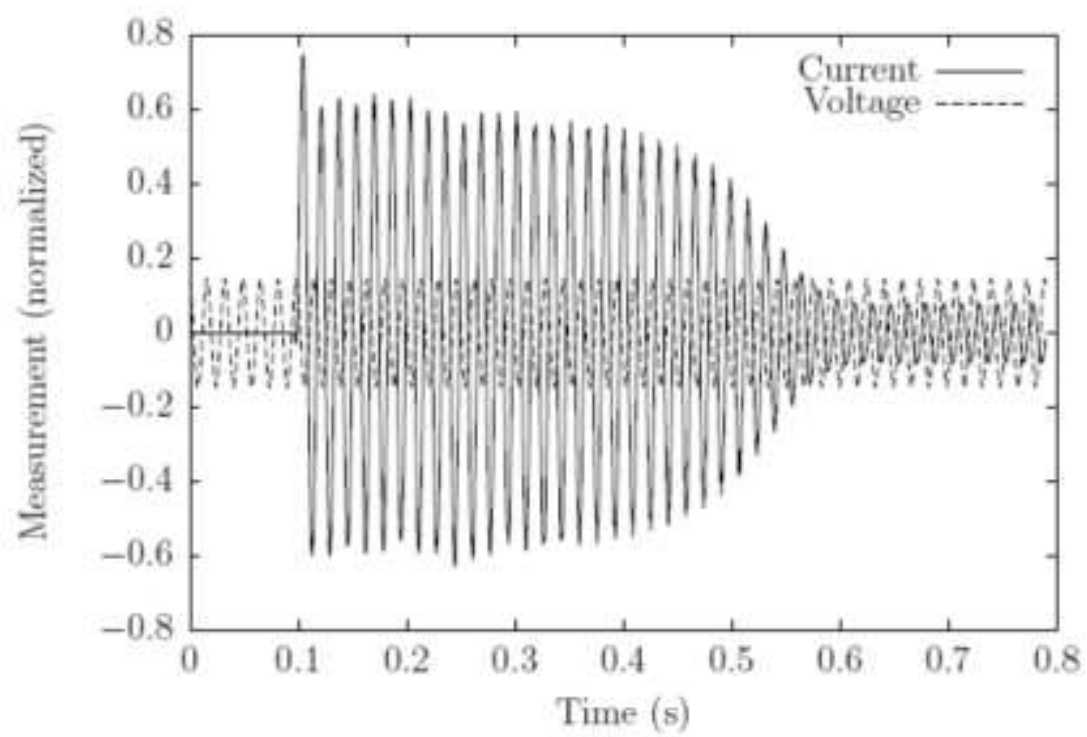

Figure 4: Raw AC voltage and current measurement captured during motor start transient [8]

In the Figure. 5 the power signal is generated from the waveforms are shown in the Figure. 4 above.

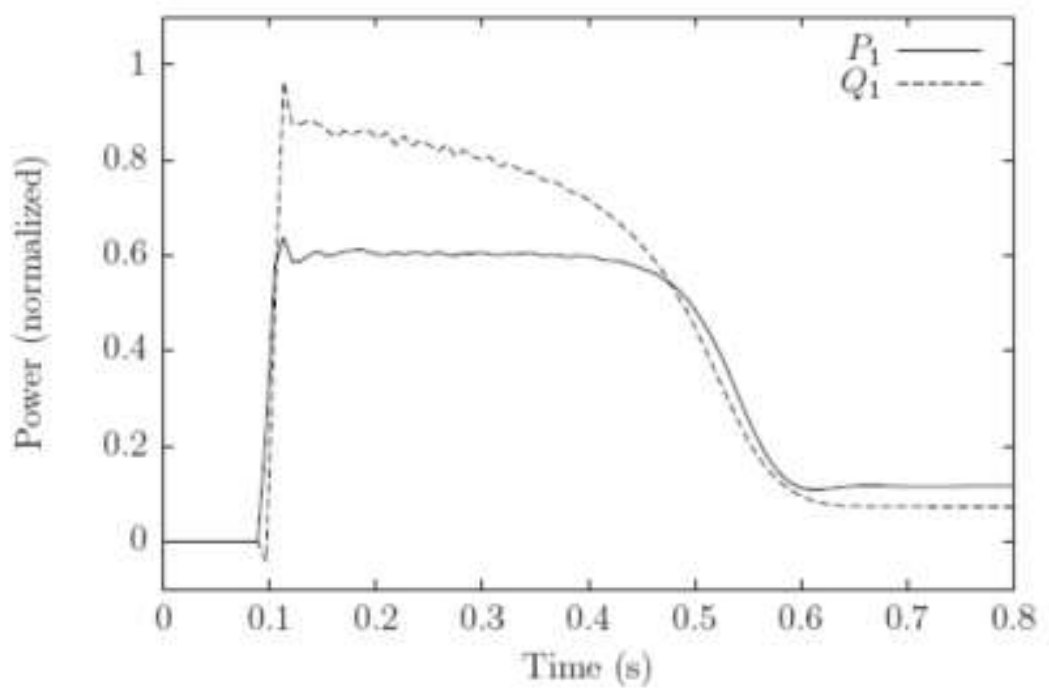

Figure5: Voltage and current signals shown in previous figure are converted into a power waveform. [8] 
Figure.6 shown the changes in the power represents turning off or on of the devices in a residential house.

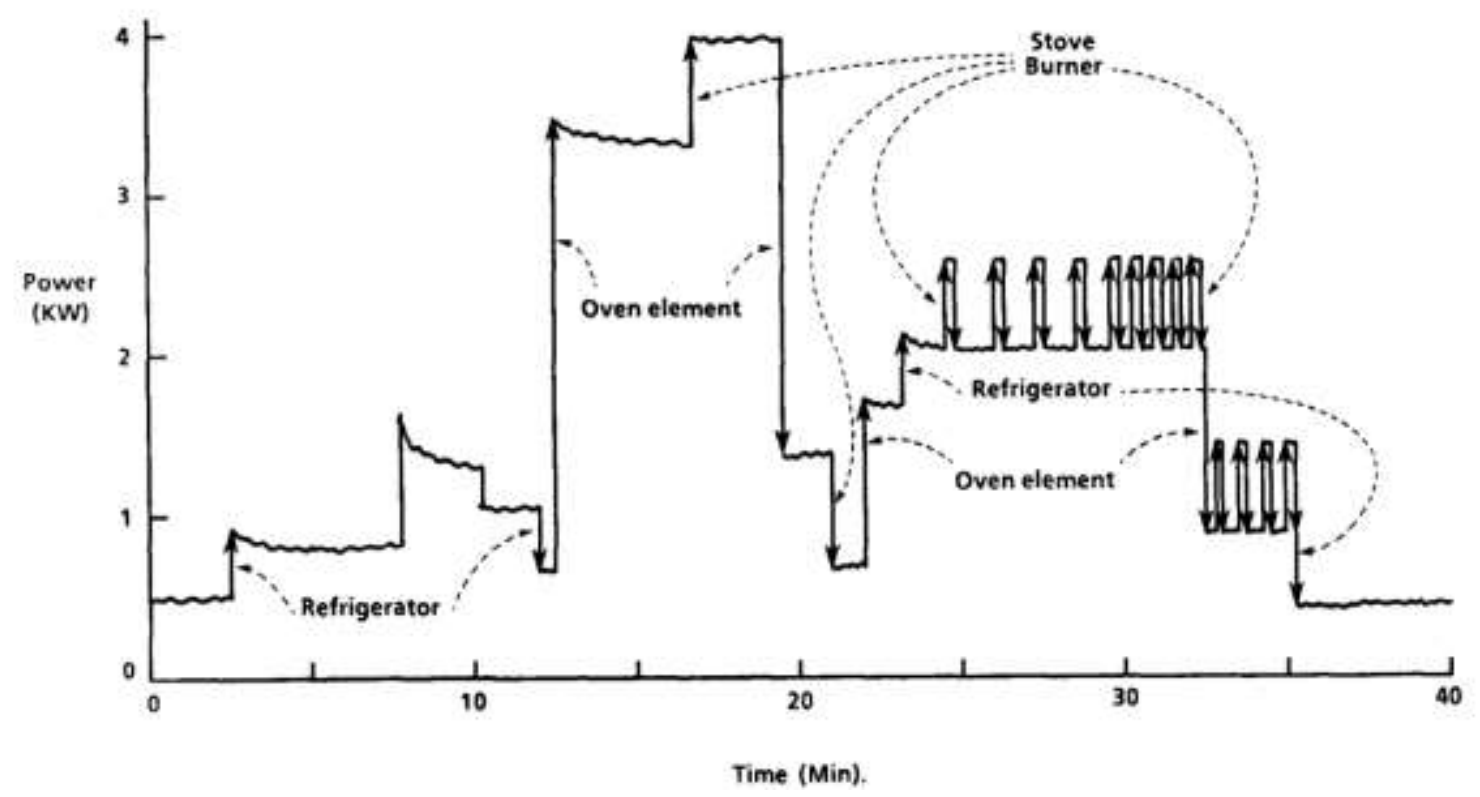

Figure 6 Power vs. time (Total load) [1]

The PQ plane is one method of power representation for appliances, where $\mathrm{P}$ stands for Active power and Q stands for Reactive Power. An example of PQ plane for several appliances in a household is shown in the Figure 7. As is obvious, resistive appliances (water heater, Incandescent light bulbs, iron) appear on the real axis and the others (like motors) that have reactive power are spread across the PQ plane. 


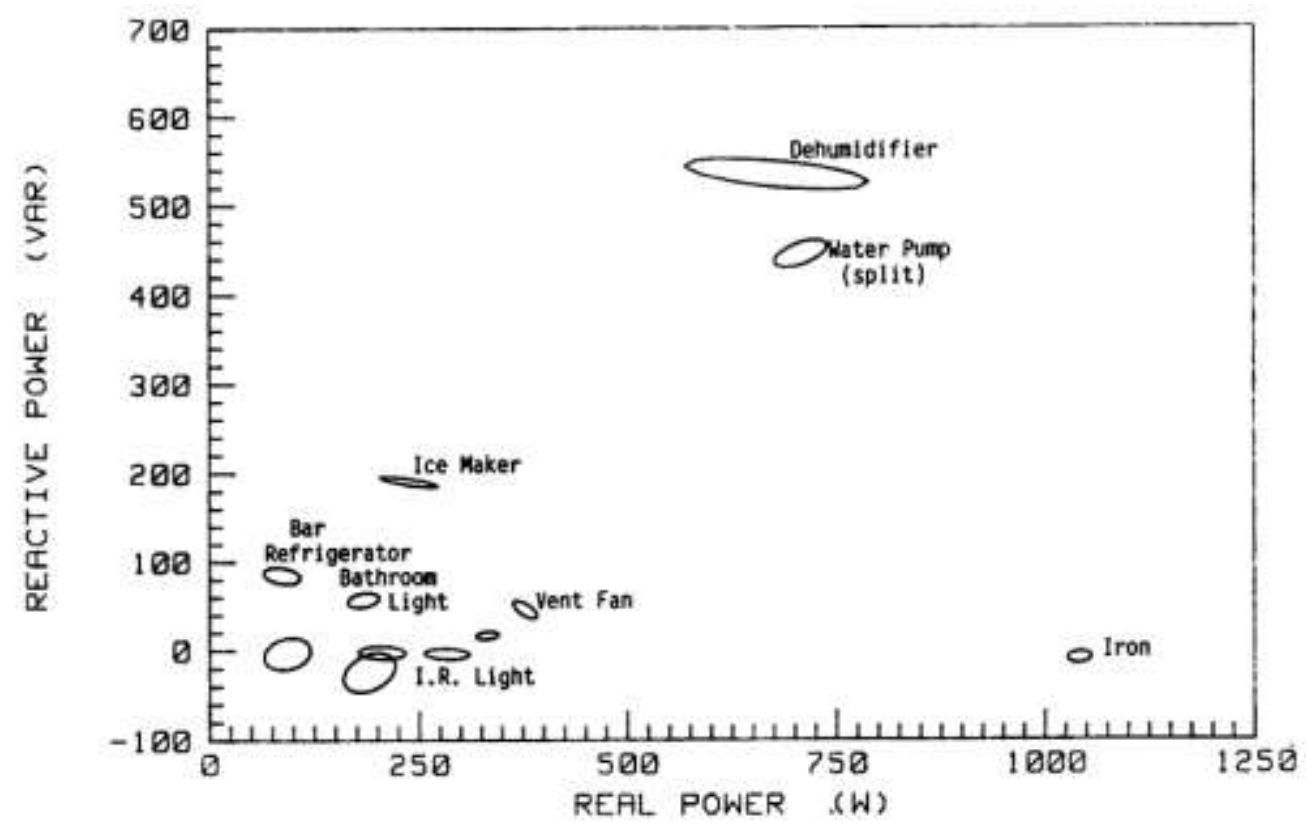

Figure 7 Normalized complex power signature space [1]

Over the past 20 years, several approaches used to analyze the power data in order to find several specifications of systems. For instance, this system can be used in several applications such as finding an engine problem in an aircraft or detect a clog in the vacuum pump in ships [9]. Another example is to diagnose problems in electrical systems for commercial buildings or equipment to detect problems as a precautionary measure before a disaster happens or even to enhance electrical audits.

Some useful applications of NILM include the following:

- Detect Elders Cognitive Problems (by keeping their privacy)

- Perform surveys of both residential and commercial energy consumption.

- Detecting drunk driving [5].

- Demand response system for use on the smart grid.

- Monitor systems on-board ships for safe systems operation with fewer sensors. [10] 
One of the important application of using NILM could be in big ships. A study was done utilizing the NILM onboard three different ships to determine its effectiveness in determining the state of mechanical systems through analysis of electrical power data with NILM. Data collected from the Auxiliary Seawater System onboard the USCGC SENECA indicate that the NILM is able to predict several faults (clogged pump inlet strainers, faulty motor/pump coupling, and fouled heat exchangers) as well as provide a backup indication of flow levels to heat loads. Data collected from the Sewage System of the SENECA indicate several metrics which can be applied to cycling systems in general to differentiate between periods of heavy usage and fault conditions. Finally, data collected from the Steering System of the SENECA as well as a yard patrol boat operated by the Office of Naval Research shows the potential of the NILM to be used as a control system without the need for separate mechanical transducers. [11] [12]

Another important application of NILM could be in Smart Homes. The results of analysis for a NILM system can identify various loads with the home and detect the condition of use for loads including of the electric power demands, names or items, time of use and overloaded capacities of loads, etc. The residents of the home can be reminded to save energy by these results. Besides, some related policies of saving energy, reducing $\mathrm{CO} 2$, health and safety care for hidden elderly and the efficiency of electric appliances can be established and planned by these results of smart home. This method requires high resolution data with high sampling rate. [4] 
One approach that is not using power signals is using the voltage and current signal directly and by analyzing V-I trajectory and also harmonics of both signals. In this way, there is more information to detect and identify loads.

Choice of load signature or feature space is one of the most essential and fundamental design choices for non-intrusive load monitoring or energy disaggregation systems. Electrical power quantities, harmonic load characteristics, canonical transient and steady-state waveforms are some of the typical choices of load signature or load signature basis for current research addressing appliance classification and prediction. The paper and research was done to expand and evaluate appliance load signatures based on V-I trajectory-the mutual locus of instantaneous voltage and current waveforms-for precision and robustness of prediction in classification algorithms used to disaggregate residential overall energy use and predict constituent appliance profiles. Therefore, wave-shape features are found to be an effective new basis of classification and prediction for semi-automated energy disaggregation and monitoring systems (see Figure 8) [13] [13]. 


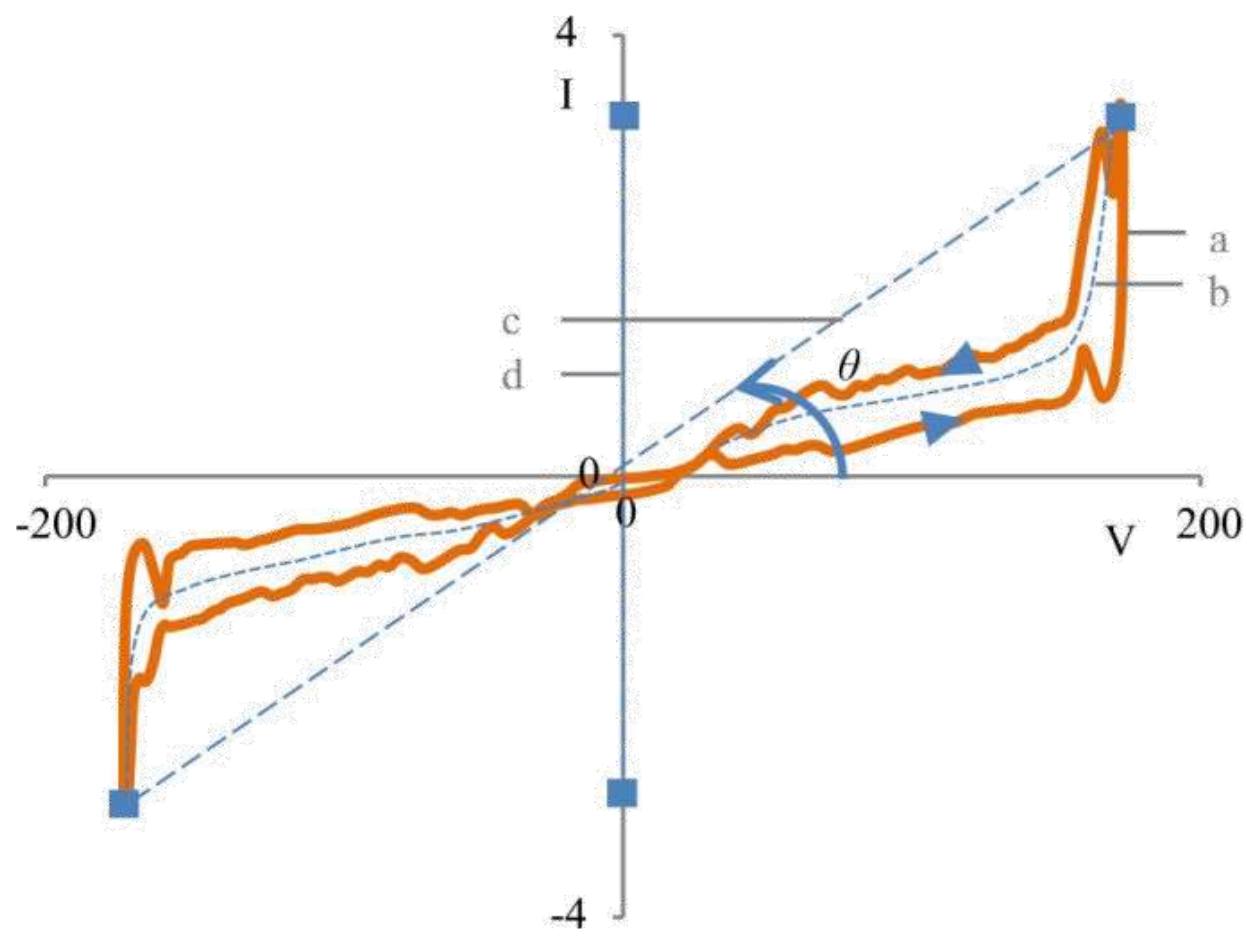

Figure 8 A graphical illustration of wave-shape metrics: (a) V-I trajectory. (b) Mean curve. (c) Reference line joining points of highest and lowest I-coordinate in the V-I plane. From [13]. 


\subsection{Outline}

The remainder of this thesis will describe the development of a prototype NILM system for residential activity analysis. Chapter 2 is about designing and developing the hardware for the prototype system, which includes data acquisition hardware as well as a means to wireless transmit the data to a data processing/analysis computer. It also includes some basic signal preparation to prepare the data for further analysis. Chapter 3 discusses the software aspect of the project, including details related to developing and finding the best

method to analyze the data recorded by the hardware. Chapter 4 presents results and discusses limitation of this project. Finally, Chapter 5 presents some ideas for future research. 


\section{System Development and Implementation}

\subsection{Cost and Limitations}

Since it is already mentioned, this system is designed to install and monitor elders' activities. Because of that, there are some limitations that should be considered in designing the system. It is important to maintain privacy of those persons being monitored, but it is also very important to ensure their safety as well. Since most circuit breaker boxes are inside houses or apartments, the system should be small enough to be installed inside the circuit breakers boxes. In this manner, no wires will protrude from the circuit breaker boxes, which is necessary due to the electrical code regulations. Due to this limitation, the system should be all installed inside and be wireless enabled.

The other limitation is the total cost. This project had budget constraints, but this same constraint will exist for any widespread deployment of a production system. Clearly, if the cost of a developed system is too high then it will not cost-effective or practical to have such a system deployed in many homes.

Another limitation is about monitoring multiple residences with one computer. In order to do that, all NILM systems in one complex should be connected to one wireless router. Then, by having only one computer connected to that router, we can gather the data and process it there. 


\subsection{NILM- Method \& Strategy}

This section outlines the development and implementation of the NILM hardware that is modified for the eldercare project, along with an associated Matlab software to detect the device status and collect the useful data for further analysis. Figure $9 \& 10$ are depicting an overview of the idea that is developed in this thesis.
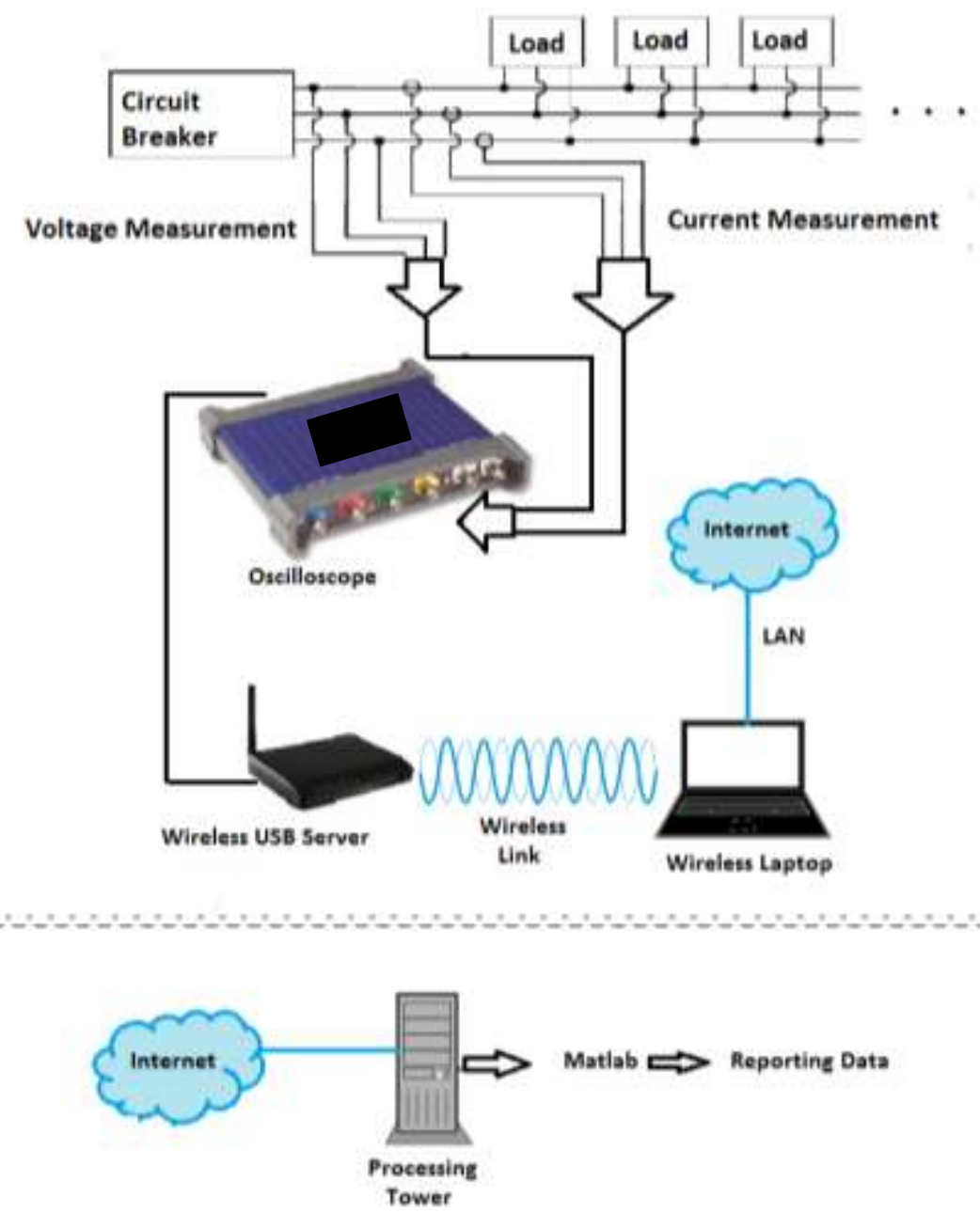

Figure 9 Eldercare House Modified NILM Architecture 


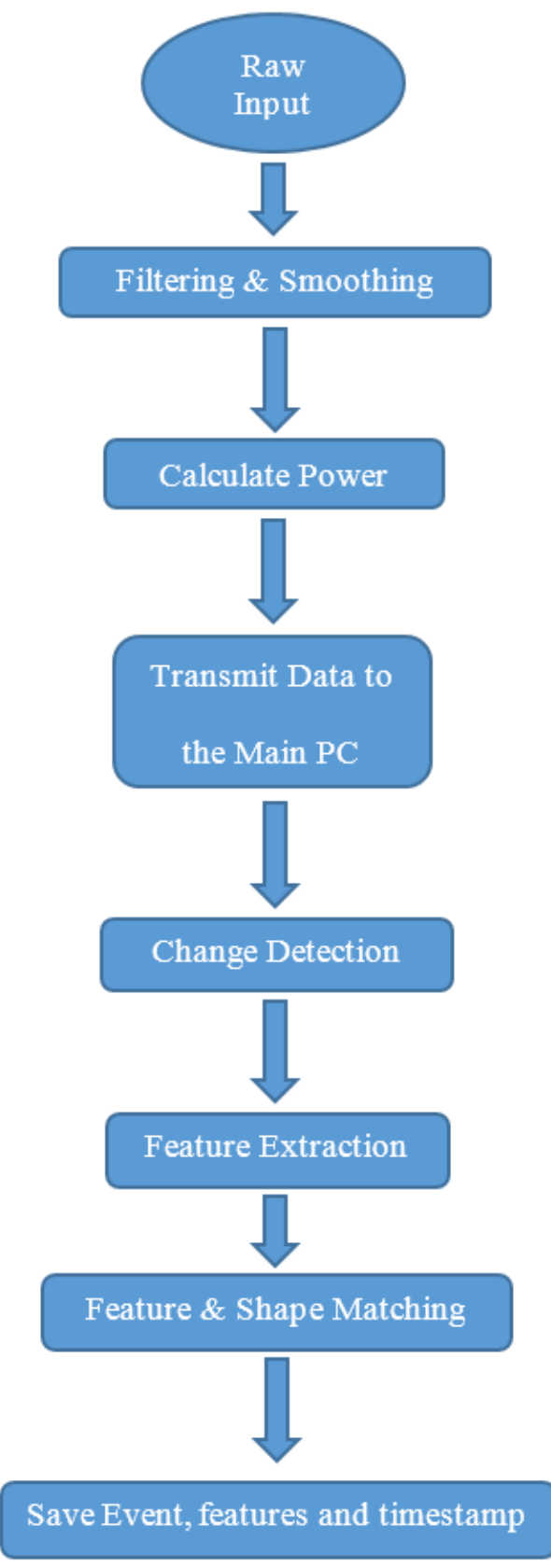

Figure 10 Overview Diagram of NILM 
One challenge in the implementation process is to find a way to make a wireless connection between the Laptop and the Oscilloscope. The main issue in this matter is about NEC (National Electrical Codes) regulations, which prohibits wires protruding from the circuit breaker box. At the same time, as a practical matter, we would prefer to avoid any wires or other accessible devices that an elder could potentially disturb or touch. This is especially true when you consider that elders may exhibit mobility or cognitive problems that might put them at a higher risk for dangerous interactions. Due to this, the NILM hardware should be separated into two parts. One part includes the current clamps or probes, an oscilloscope, voltage dividers, a USB wireless server, and a power supply for the oscilloscope and wireless server. The other part consists of a laptop with LabView software installed, which should be connected to the Internet to report the data to the data processing computer for further analysis.

\subsection{Developing Hardware Framework}

In this section, the selected hardware and the procedure to implement the whole system is discussed. As previously mentioned, we need to construct the hardware in three parts. The first part is comprised of the measuring devices and tools, which includes the current transformers, voltage divider, oscilloscope, wireless USB server, and some other peripherals to connect these devices properly together. The second part is the data logging and signal preprocessing laptop. LabView is installed on this system to set up the oscilloscope and then process the signal, calculating the active and reactive power $(\mathrm{P}, \mathrm{Q})$ 
and also to find the harmonics for each phase. The third part includes a powerful and fast computer to run algorithms to detect the pattern of appliance usage from the data logged by the oscilloscope and preprocessed by LabView. Figure 11 illustrates the first two part in a diagram.

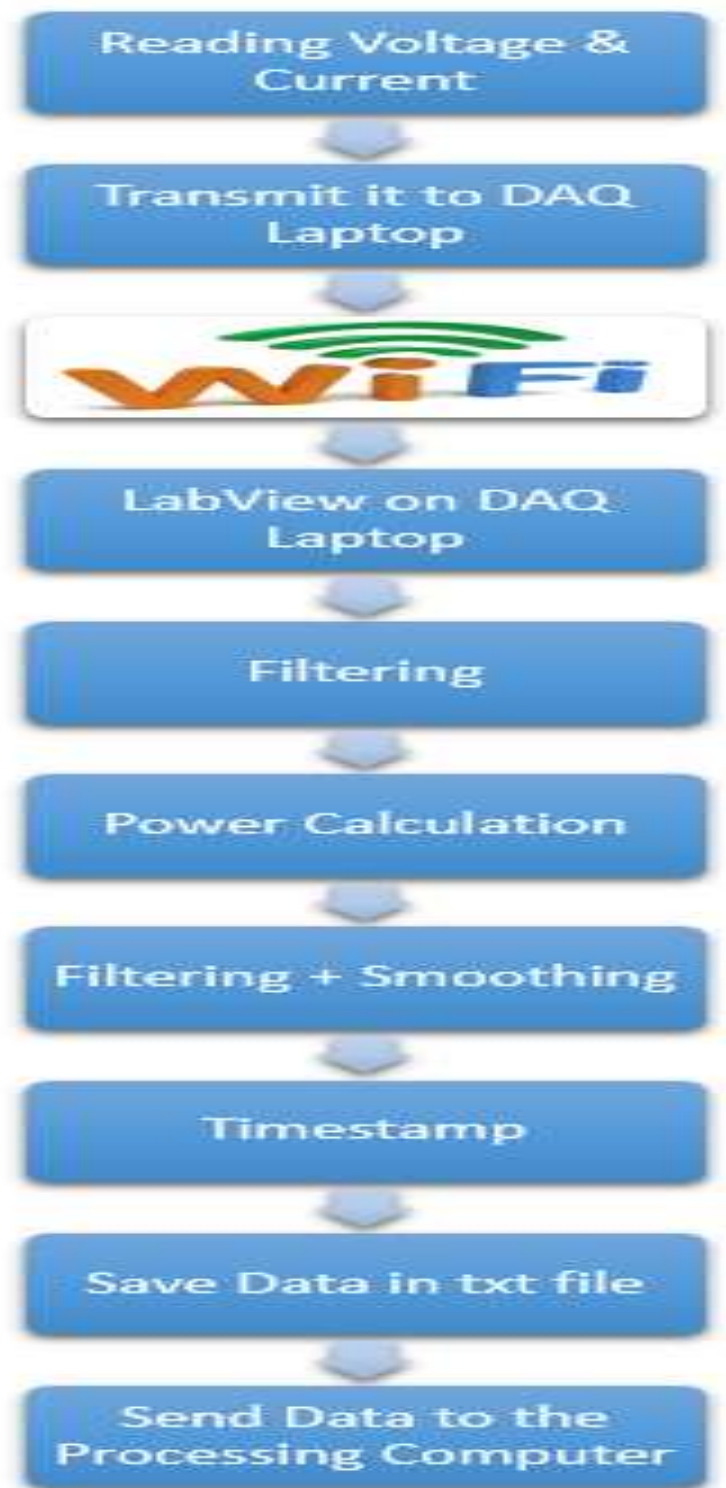

Figure 11 Data Acquisition \& Signal Preprocessing Part of NILM 


\subsubsection{NILM Hardware Framework- First Part}

It is very important to have accurate data in this project. Because of that, we used an oscilloscope to capture accurate and reliable data. Additionally, we would like to perform frequency analysis, but the Nyquist criterion is insufficient and we need to capture the voltage and current signal with more than 2 times of the main signal frequency in order to overcome noise and aliasing problems. We selected the Pico Oscilloscope 3404A. This oscilloscope is a general purpose 8-bit mixed-signal oscilloscope (MSO) that combines fast sampling rates with class-leading deep buffer memories [14].

Initially, we tested an ELITEpro XCTM Energy Logger (Figure 12) which also has a builtin wireless option. The problem encountered with this unit was that we were not able to export the data to another software. We also found that the precision of the sampling with this device was inadequate. Since there is a need to transfer the data to Matlab or LabView and this tool only works with its own software (no drivers for Matlab or LabView are available), it could not be used for this research. Furthermore, it only could get 2 samples (maximum) per second, and this is only sufficient for energy auditing and some basic analysis. Since this project is trying to detect the on/off event signatures, two samples per second is woefully inadequate. 


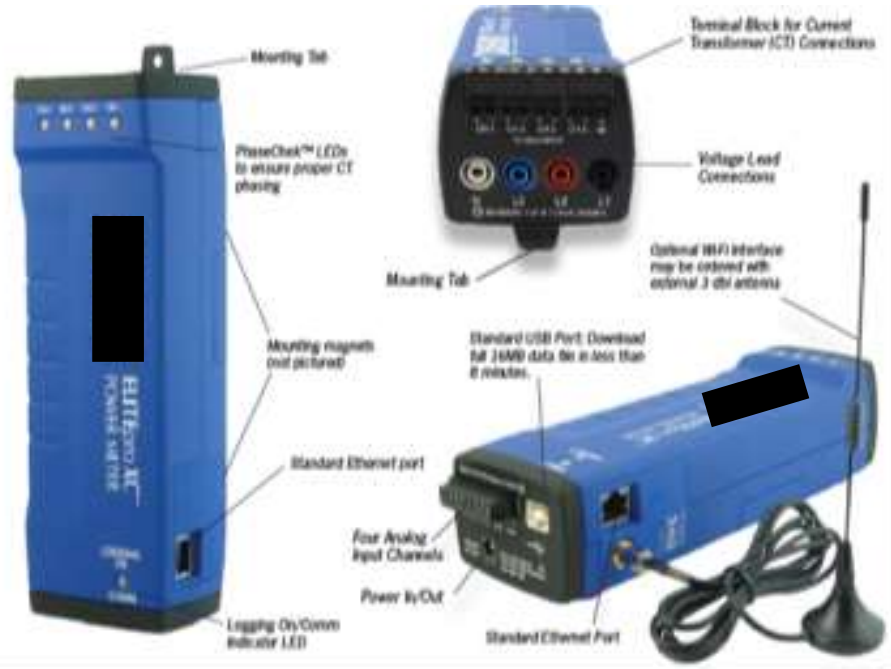

Figure 12 ELITEpro XC ${ }^{\mathrm{TM}}$ Energy Logger [15].

Since we need to measure the currents of each phase without having to make physical contact with wires (or to disconnect it for insertion through the probe), we used normal current clamp transducers for this purpose. The wires are simply surrounded by the clamps without electrical conduction between them.

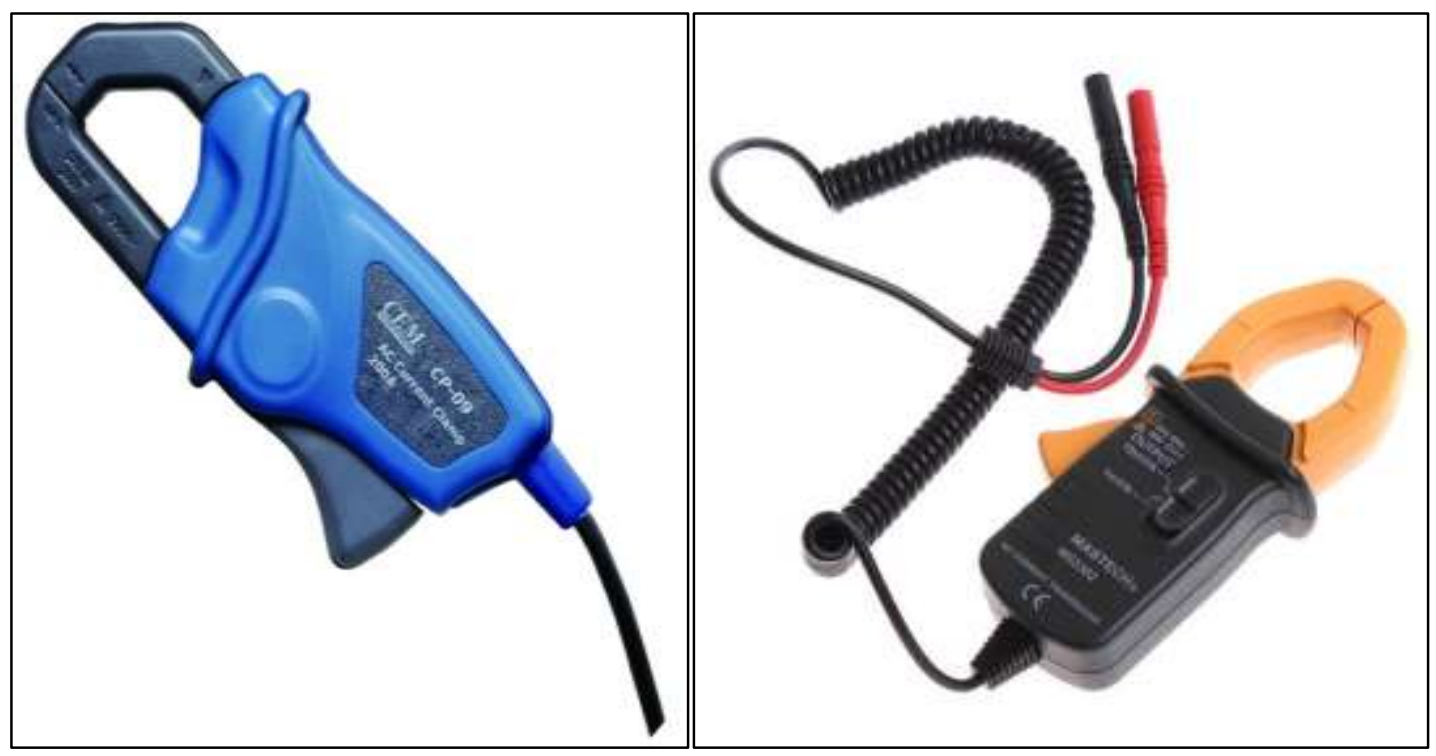

Figure 13 Left: Pico current clamp 1mV/A, Right :Mastech current clamp 10 mV/A 
We tried several different models of current clamps and selected the Mastech MS3302 current transducer (see Figure 13, right). The output of this clamp is $10 \mathrm{mV} / \mathrm{A}$ and it supports up to 400A. This makes it a really good candidate for this project because we need to measure small changes in current, and comparing with other choices in the same price range its output is 10 times greater. The only problem that we faced is high nonlinearity of the clamp at the higher current range. Regarding that issue we used Pico TA138 - 200A (see Figure 13, left), the sensitivity of the clamps is $1 \mathrm{mv} / \mathrm{A}$ which is not high as the last one but the sensor keeps its linearity even at the higher current range. The only limitation we had is the budget and cost of the tools. Due to that we were not able to purchase very high quality current clamps. The price for some higher quality current clamps start at least 5 times the price of the current clamps chosen for this project. In Figure 14, the clamps are installed on 3 phase system circuit breaker box.

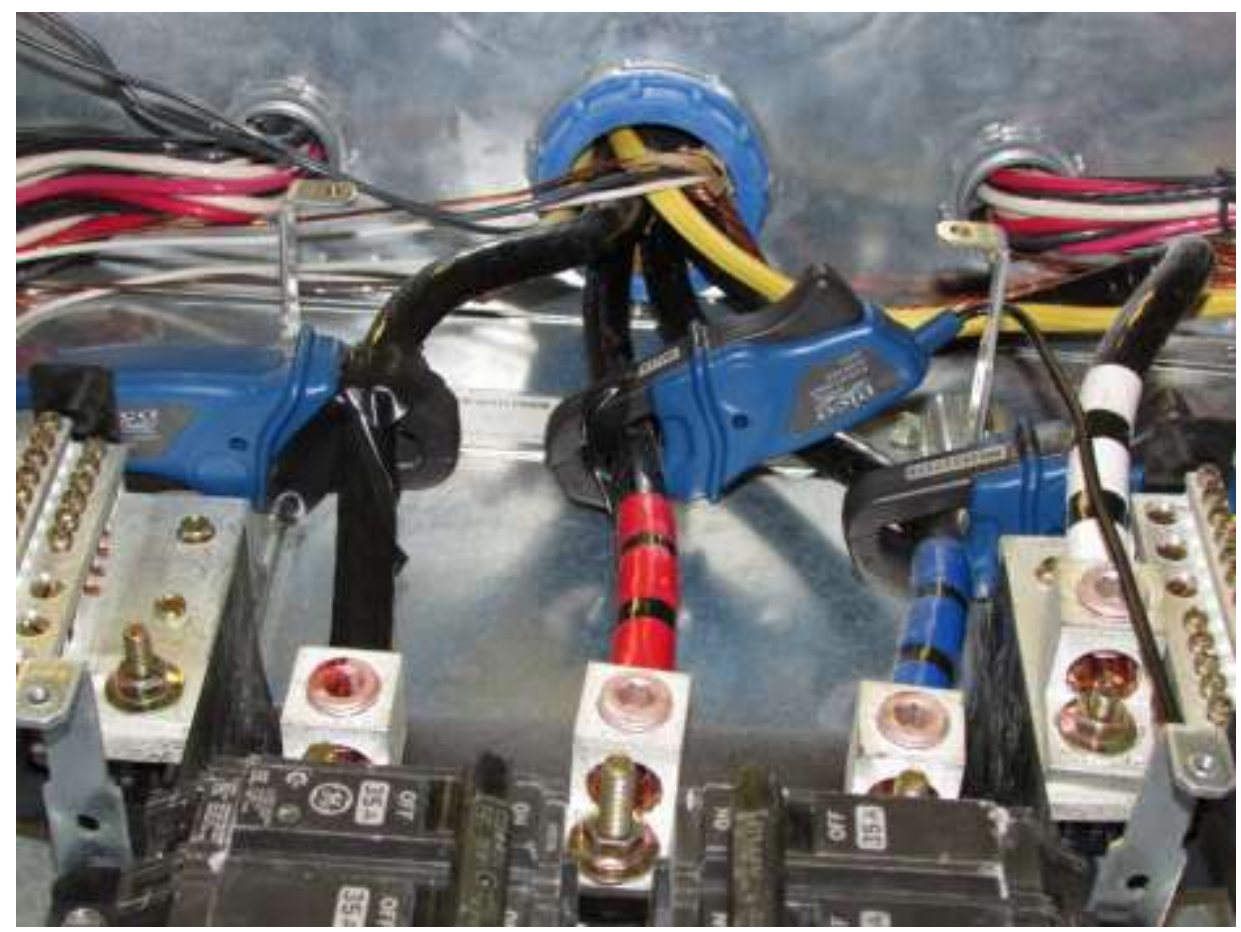

Figure 14 Current Clamps Installed inside the Circuit Breaker 
Another important element in this part is the wireless USB server. As already discussed, there is no way to transfer the data from the oscilloscope to the laptop with wires due to NEC (National Electrical Codes) regulations (and, as also mentioned, we must consider safety concerns particular to elders). It is also important to have all of the data from multiple rooms available to one computer, making it more efficient to record and store the data. Because of this, wireless capability is necessary and should preferably connect directly to routers. Given that wireless Internet connectivity is nearly ubiquitous, it would be a good idea to use wireless routers as main node of connections. On the other hand, keeping the private data and sending securely to the server is also important. Because privacy and security of data are important, using wireless router and USB server make it possible to encrypt the data by WPA or WEP encryption.

Several brands of USB servers were tried to find the right one. The one that we selected is "Hawking Technology Wireless-N Multifunction USB Printer and Device Server (HMPS2U)" that shown in the Figure 15.

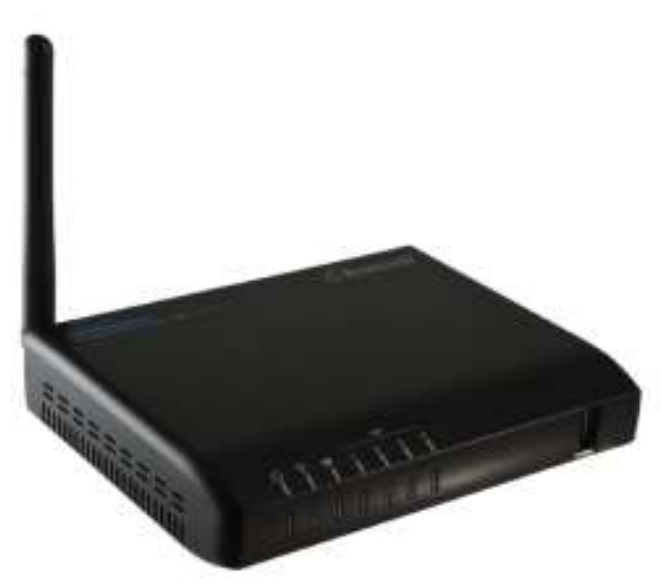

Figure 15 Wireless USB Server [16] 


\subsubsection{The NILM Preprocessor}

The NILM collects and processes two input data streams - line voltage and aggregate current. Using these two quantities, software on the NILM can perform a number of important tasks, including:

- real-power computation

- reactive-power computation

- current-harmonic computation

- power-quality analysis, and

- load identification.

The first four tasks listed above are performed by the NILM's preprocessor. This software is implemented in LabView and performs several calculations such as calculating RMS of signals, active power and reactive power, harmonic analysis, and power quality analysis. In Figure 16, it shown a summary of the LabView and Power Suite Toolbox inputs and outputs. This section provides a qualitative description of the tasks performed by preprocessing, and it also describes how the user should interpret various preprocessed outputs in typical monitoring situations. 


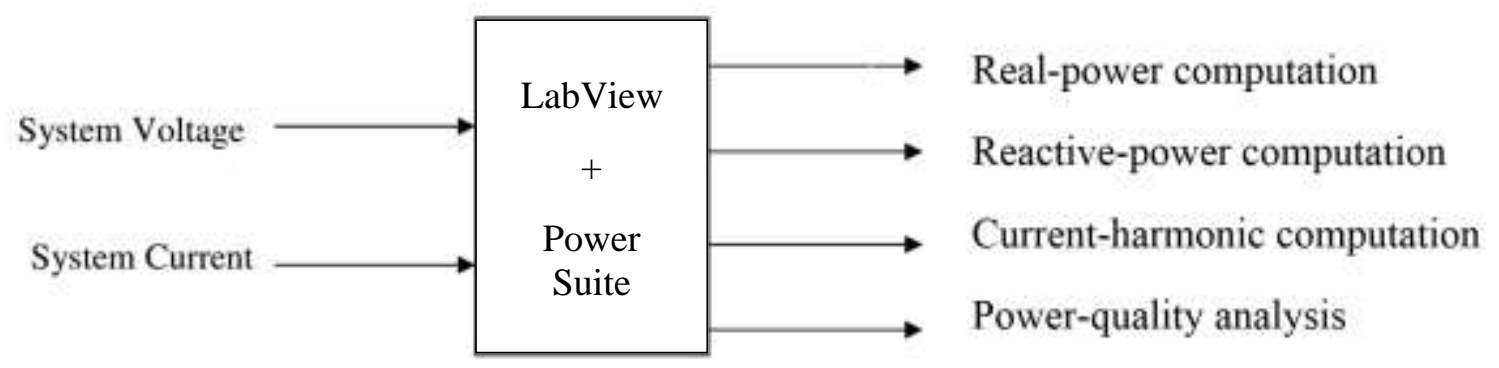

Figure 16 Preprocessor block

Assuming minimal distortion, the line voltage can be approximated as either a pure cosine or as a pure sine. In this example, it will be assumed that the line voltage is a cosine and it can be shown as below.

$$
V(t)=V * \cos (\omega \mathrm{t})
$$

In general, the current drawn from such a source will contain a number of harmonics, i.e.

$$
\begin{aligned}
& i(t)=I_{1} * \cos \left(\omega \mathrm{t}+\varphi_{1}\right)+I_{2} * \cos \left(2 * \omega \mathrm{t}+\varphi_{2}\right)+I_{3} * \cos \left(3 * \omega \mathrm{t}+\varphi_{3}\right)+\cdots= \\
& \sum_{k} I_{k} * \cos \left(\mathrm{k} * \omega \mathrm{t}+\varphi_{k}\right)
\end{aligned}
$$

Equation 2-2 above highlights a key fact that the current drawn at any one harmonic frequency is characterized using two quantities, namely an amplitude $I_{k}$ and a phase shift $\varphi_{k}$. The phase shift represents any offset from the line voltage, and the amount of phase shift is strongly dependent on the type of load. Purely resistive devices such as heating elements draw currents that are completely in phase with the voltage, i.e. $\varphi_{1}=0$. By 
comparison, a purely inductive or capacitive load will draw a current that is 90 degrees out-of-phase with the line voltage, i.e. $\varphi_{1}= \pm \frac{\pi}{2}$. These loads are often referred to as purely reactive. Many common devices such as motors consist of both resistive and reactive elements. In those cases, the magnitude of the phase shift will lie between 0 degrees and 90 degrees. The sign of the phase-shift depends on whether the load is either inductive or capacitive in nature (negative shift for inductive load, positive for capacitive load).

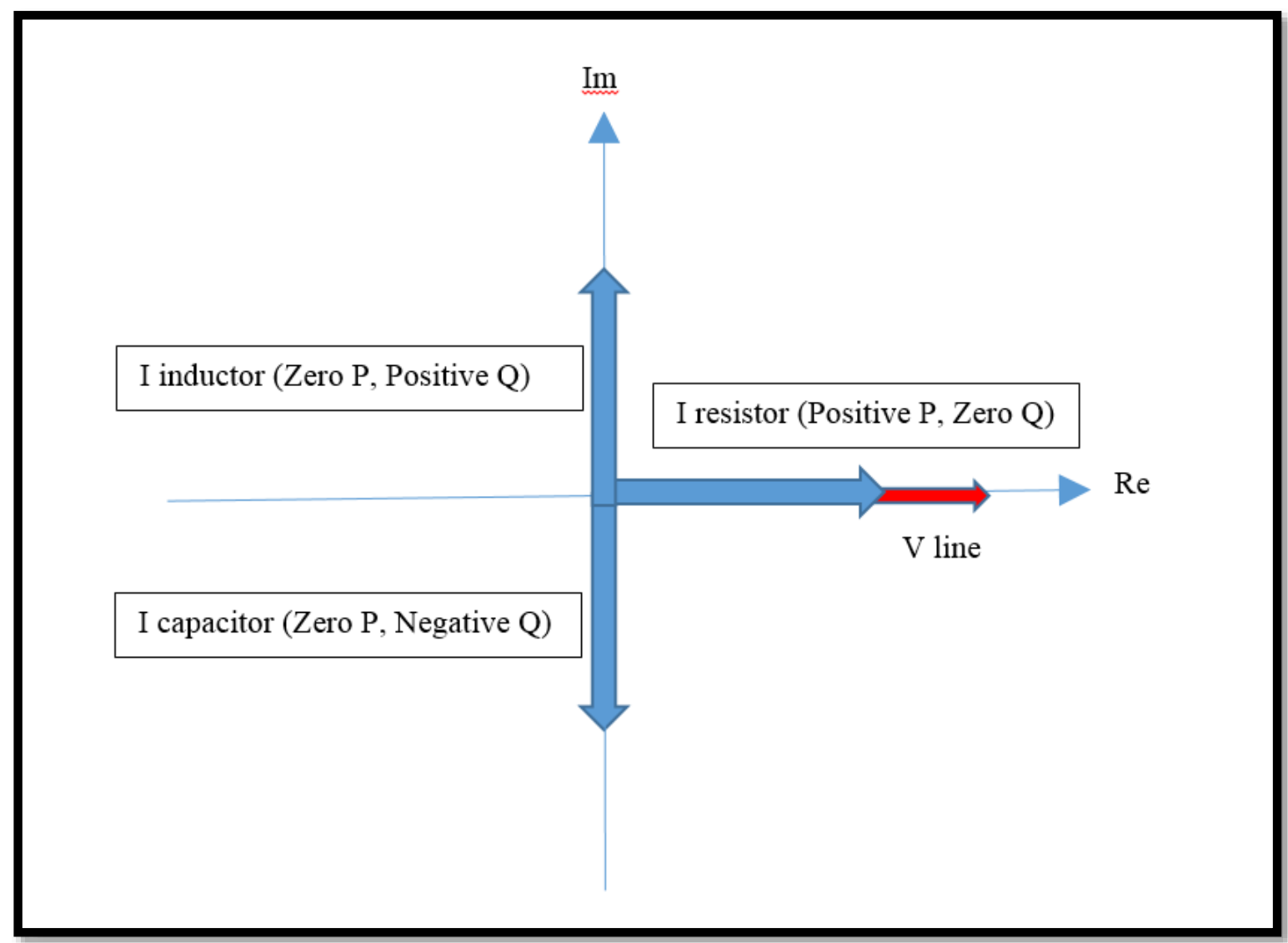

Figure 17. Voltage and current phase relationships for 3 components in $P Q$ plane. 
The primary function of the preprocessor is to estimate the real and reactive power drawn from the ideal single-phase source presented in Eq. 2-1.

By definition, the real power is

$$
P_{1}=\frac{1}{T} \int_{T} V * \cos (\omega t) * i(t) d t
$$

and the reactive power is

$$
Q_{1}=\frac{1}{T} \int_{T} V * \sin (\omega t) * i(t) d t
$$

These calculations for active and reactive power and along with the harmonics are done in the Power Suite Toolbox in LabView. 


\subsubsection{Power Calculation}

The NILM uses measurements of voltages and currents to produce estimates of real and reactive power consumption (henceforth referred to as $\mathrm{P}$ and $\mathrm{Q}$, respectively). For a single phase system, the NILM is supplied with the voltage from line to neutral and the current flowing to any loads downstream of the monitoring point. Real power $(\mathrm{P})$ is produced by current which is in phase with voltage and reactive power $(\mathrm{Q})$ is produced by current which is 90 degrees out of phase with voltage. It also estimates the higher harmonic content of current to produce the first three odd harmonics of real and reactive power (referred to as $\mathrm{P}_{k}$ and $\mathrm{Q}_{k}$ for the $\mathrm{K}_{t h}$ harmonic of $\mathrm{P}$ and $\mathrm{Q}$, respectively). If the same sinusoidal voltage is applied to a resistor, capacitor, and inductor, the NILM would produce positive P with zero $\mathrm{Q}$ for the resistor, positive $\mathrm{Q}$ with zero $\mathrm{P}$ for the inductor, and negative $\mathrm{Q}$ with zero $\mathrm{P}$ for the capacitor (see Figure 17). The convention of using a power angle measured in the counter-clockwise direction from the real axis is employed, so that the power angle for an inductor is +90 degrees while the power angle for a capacitor is -90 degrees [17]. For a more in-depth description of the NILM calculations used to determine P and Q, see [20].

Prior to completing a hardware prototype for capturing voltage and current waveforms, the NILM preprocessing software was given one signal pair from a waveform generator. All tests used $60 \mathrm{~Hz}$ as the principle frequency. Channel 2 was chosen to lead channel 1 simply because current generally leads voltage in predominantly inductive circuits. These tests were run and preprocessed data were recorded for each. 


\subsubsection{NILM Hardware Framework- Second Part}

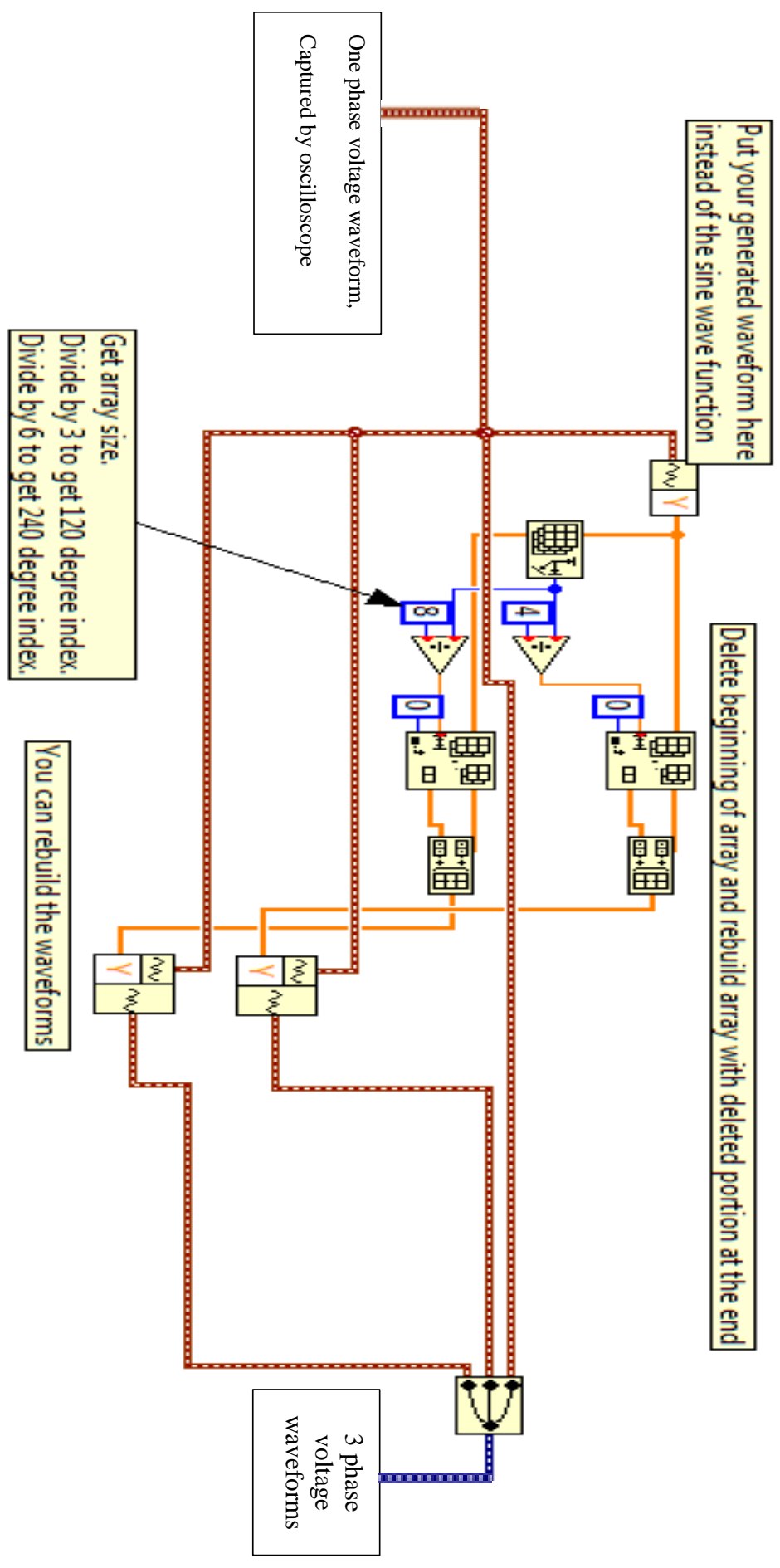

Figure 18 Simulating voltage signals for 3 phase system based on one phase data 
In Figure 18, the NILM hardware can measure and capture only one phase of the voltage because it only uses one oscilloscope having four channels, and three of the channels are used to capture and measure currents. If the NILM device is installed on a two phase system, then the second phase is 180 degrees out-of-phase from the first, as is typical in residential 220V service in the United States. (Note that 220V service in the United States is typically referred to as " $220 \mathrm{~V}$ single phase", but it is really two hot wires from a centertapped transformer that are naturally 180 degrees out-of-phase - thus our usage here.) Given the limitation of our hardware to capture only one phase of voltage, in order to deploy it on a typical residential installation, the second phase is simulated by taking the data of the first phase, cutting one-half of a cycle at the beginning of the data, and then pasting it at the end of the same array to produce a signal with a 180 degree shift. Simulating the second phase in this manner can be useful to save hardware implementation costs. Alternatively, if we wanted to measure all phases of voltage and current we would need to have two oscilloscope or an oscilloscope with 6 different channels. If the NILM device is installed on a three phase system, then the difference between each phase is 120 degrees. In order to deploy the system in this situation with the data of only one phase, the easiest way is to divide one cycle of the array data into 3 equal pieces and then (cut and) paste either $1 / 3$ or $2 / 3$ of the cycle to the end of the data to achieve phase shifts of 120 or 240 degrees, respectively. This method may cause a little effect on the signals, but it is not important since most of the electrical device on/off events are changing the current signal and not voltages. 


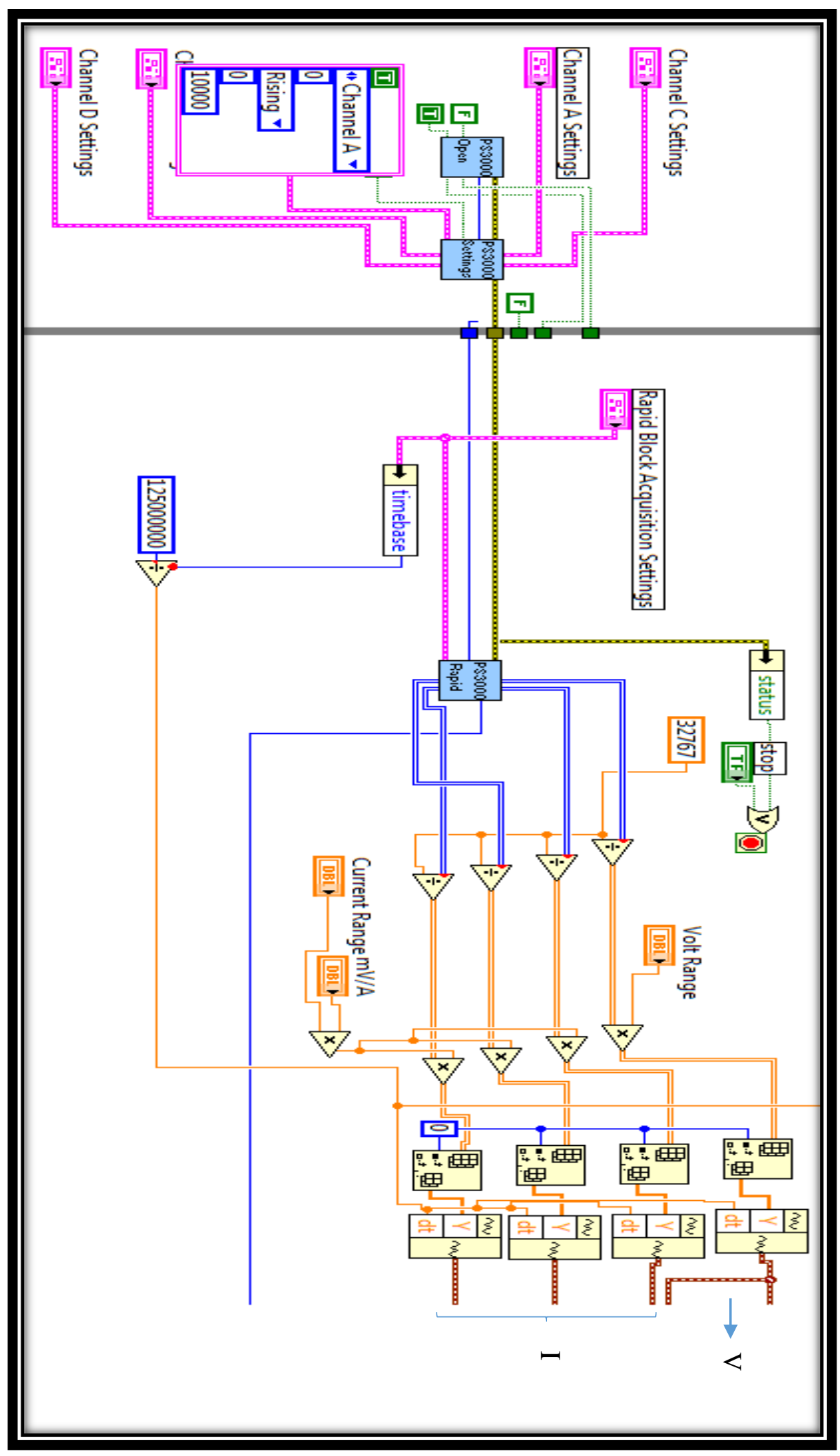

Figure 19 Oscilloscope Setup in LabView+ Main Signal calculation 
In Figure 19, the oscilloscope setup and value calculation section is shown. Configuration of some parameters is required to start and run the oscilloscope. Some setup values include setup information for each channel such as channel number, time division, volt division, AC/DC setting and offset correction. Triggering setting must also be configured such as the time base, sampling time, synchronizing trigger channel, USB power setting and number of samples in each time base.

Since the values returned from the oscilloscope are in binary format and the scale is the ratio of the full scale (which is 16 bits), we can convert them to volts (or millivolts) easily as in Eq. 2-5.

$$
\text { Channel value }=\frac{(16 \text { bit out value from Oscilloscope })}{32767} * \text { Volt division }
$$

The formula to determine the time interval requires time base and number of samples in that time base. We can then calculate the time interval between the samples and convert it to seconds (or milliseconds) with Eq. 2-6.

$$
\text { Sample }_{\text {interval }}=\frac{(\text { timebase }-2)}{62500000}
$$




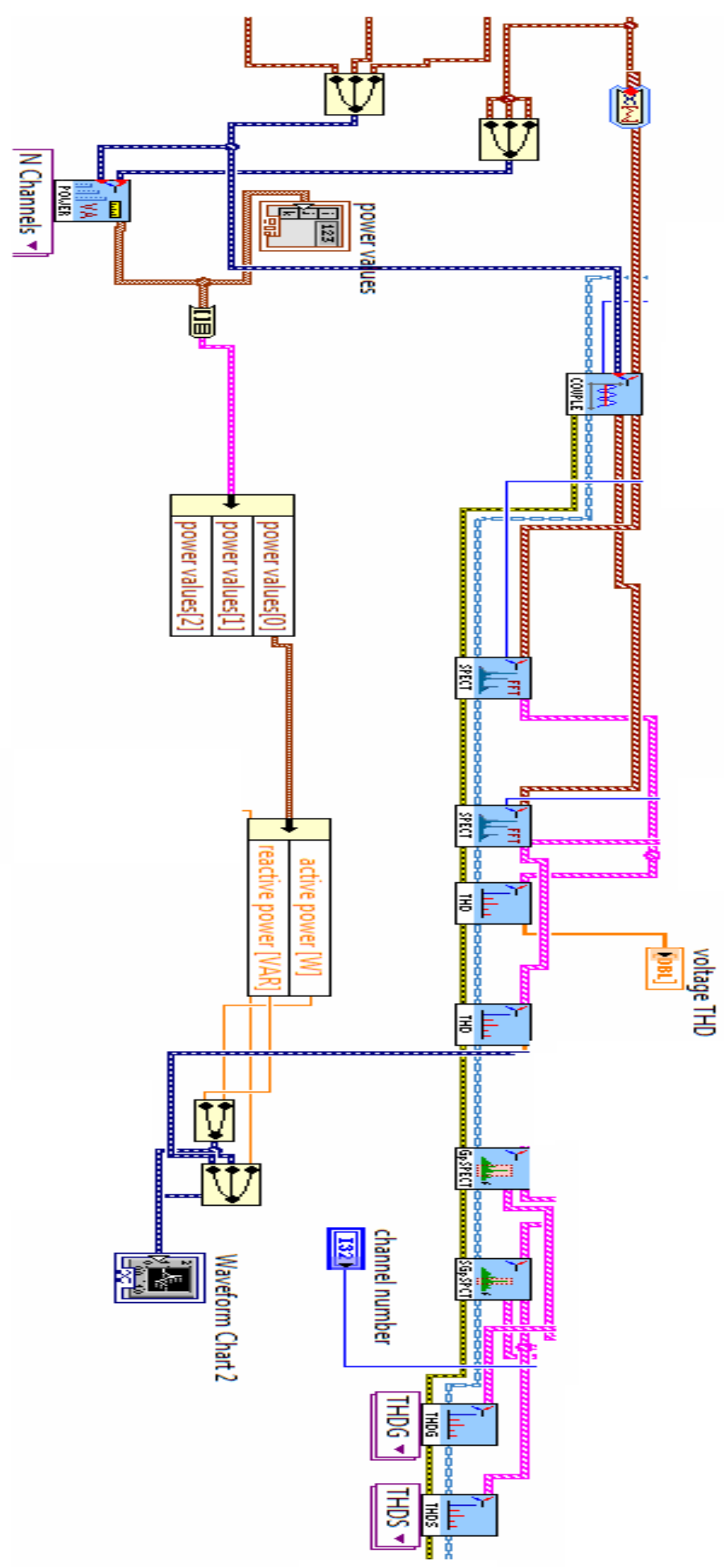

Figure 20 Power Suit module in LabView

Figure 20 illustrates power calculation for both active and reactive part in LabView. 
We also need to save data from each day in separate files, so Figure 21 illustrates a process for labelling of each text file with the date of the recorded data. This process also incorporates the timestamp (time of day) of the data into the filename. Since these data are analyzed with Matlab, the format of the time should be in a way that Matlab can detect it easily, so the easiest way is to represent the time in number of second since midnight. Each day starts at 1 and ends at 86400 seconds. For instance the file names and path that is produced by this module is like "2013-8-21.txt" which represents the day that signal is being sampled.

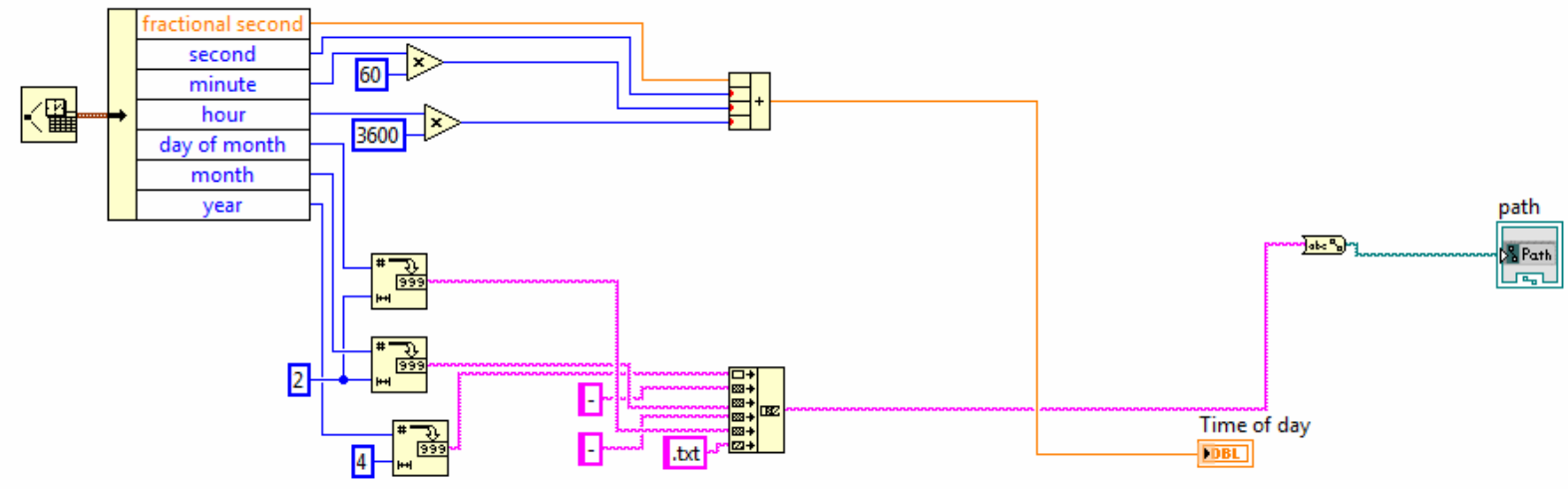

Figure 21 Automatic File path maker-LabView Module 


\subsubsection{NILM- Third part}

The data processing/analysis portion of the hardware includes a powerful computer with Matlab in order to process the data and find the device status. Ultimately, in our application, this computer could also use automated algorithms to detect cognitive problems arising in the residents being monitored via NILM.

At first, the system was tested to see if we could process the data with LabView in real-time and get results. Among the techniques used to detect the signals, one of the important ones was detecting the power signature of appliances in the LabView software. Some codes were developed to run within LabView, but many limitations were encountered, including:

- low voltage resolution of the power signal capture

- low sampling rate of the system

- lack of sufficient computational power on the LabView laptop, and

- LabView does not readily allow development of non-real-time algorithms to achieve the detection we need.

A screenshot of some developed LabView code is shown in Figure 22. This module detects the on/off events from the main signal by using an average and sliding window on the power signals. 


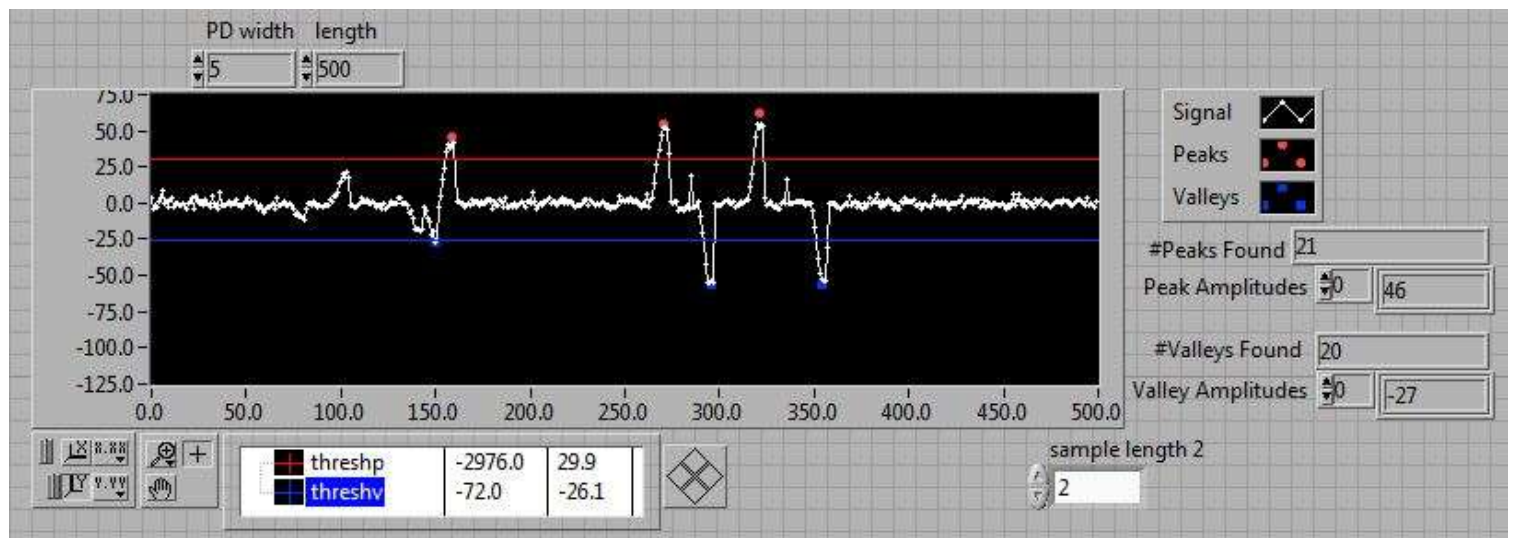

Figure 22 Sliding average window on power signal detecting events in the real-time

As mentioned, we faced a problems regarding computing power since it is necessary to deploy a small laptop in the resident's home (to minimize intrusion). This laptop is used to record the data, do some filtering and smoothing on the signal and calculate the power signals. It is not able to handle a lot of calculations. The other issue is about having Matlab on site on the laptop. Since are target for deployment (TigerPlace) is off-campus, there was no access to the network-license of Matlab there. Last and not least is about LabView; LabView sends commands to the oscilloscope to capture the signal and then return the value back to LabView. If a calculation starts and stops in the laptop, it will affect the sampling rate drastically, and in some cases the data acquisition can be stopped for several milliseconds. Due to the all mentioned reasons above, it was decided to let the laptop just sampling and preprocessing the data, and then transferring the all preprocessed data to the powerful computer in the lab to take care of the rest of the analysis. Consequently in the final project the third part of the NILM system is considered as a powerful and fast computer with Matlab installed in it to run the algorithm for event identification. 


\subsection{System Installation}

Typical NILM installations in the past involved the acquisition of voltage and current data through the insertion of transducers in a distribution panel or controller which required shutting off the power and installing the system. Since our NILM system requires power for the oscilloscope and also the wireless power supply, an extra breaker is installed in the circuit breaker box in order to provide maximum safety. Obviously, the amount of room available inside the circuit breaker is limited, so we need to minimize the required space for elements in the system; accordingly, one power supply can be used for both the oscilloscope and wireless adaptor. In our particular case, the power supply of the wireless adaptor can provide power for the adaptor, and the oscilloscope can be configured through the software (LabView) to run from USB power (provided from wireless adapter). Our system will be connected to ground via the ground port of the oscilloscope. This connection is made direction to the ground bus inside the circuit breaker box. Figure 23 and 24 shows one installation of this prototype. 


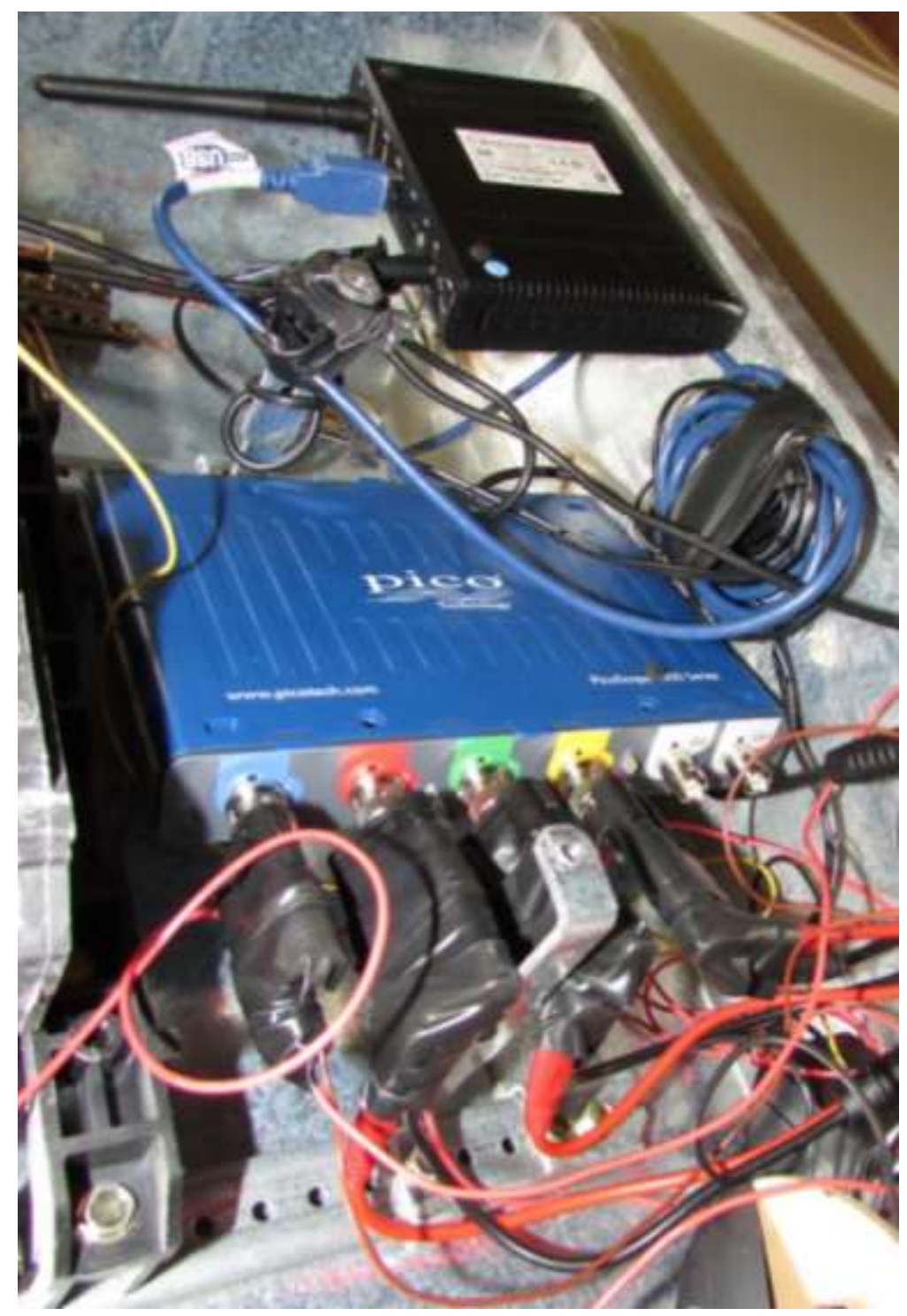

Figure 23 Oscilloscope and wireless USB adaptor 


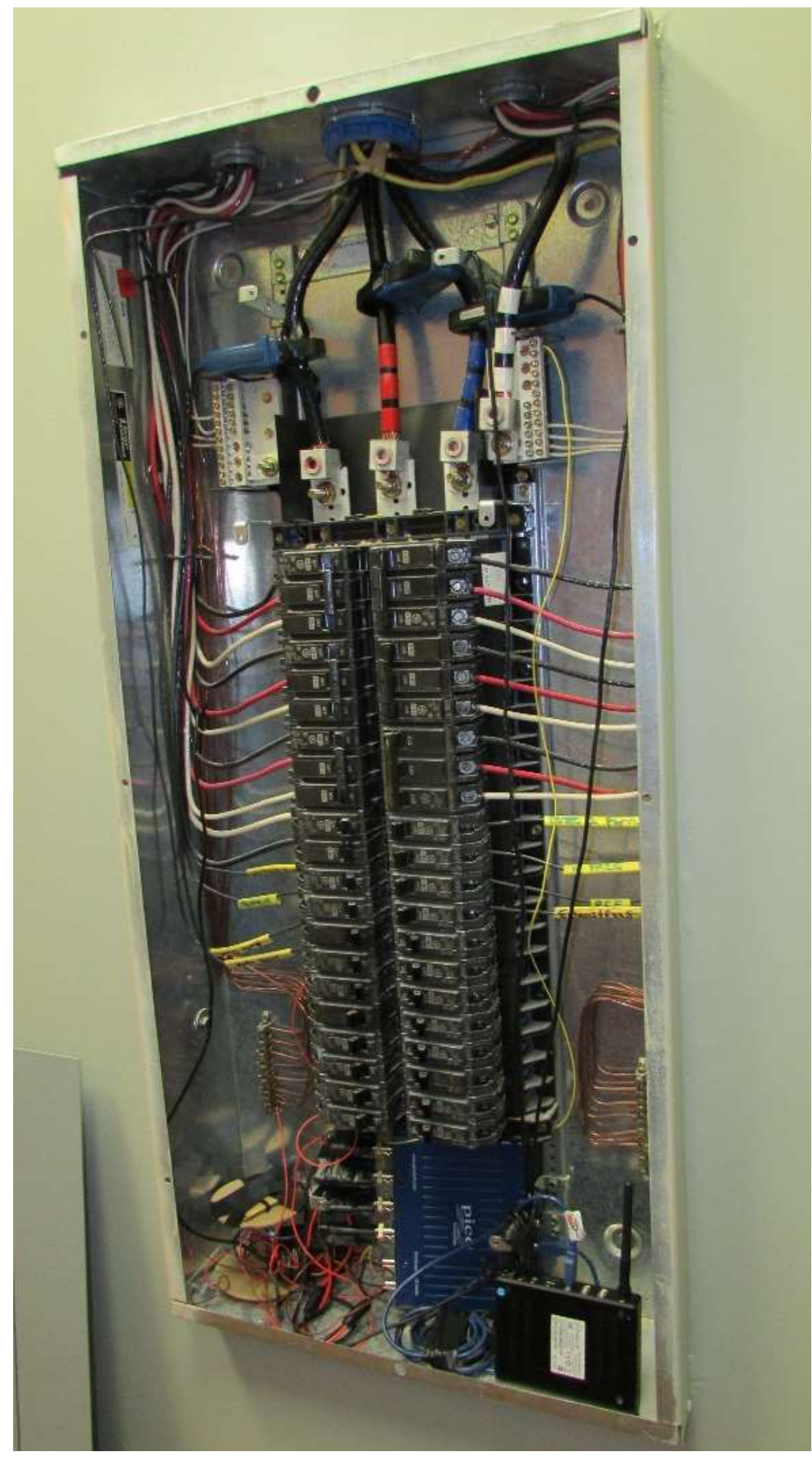

Figure 24 NILM wireless data Acquisition installed inside the circuit breaker 


\section{Detection Algorithm Development}

\subsection{Real-time detection}

In this section, the method that deployed to detect the device status in LabView is discussed. The first thing that is necessary is to detect the right signal and detect the right changes in the whole power signals. In order to do that, a real time averaging module is developed with 4 knobs to change the threshold values. It finds the average for every 5 samples and then when new sample value arrives, it is compared with the average. If it is not in the defined range it would be detected as an event and then the code measures the amplitude of the peak, the time, the duration of the event, and some other features. Figure 25 depicts both the block diagram and main frame of the module in LabView. 

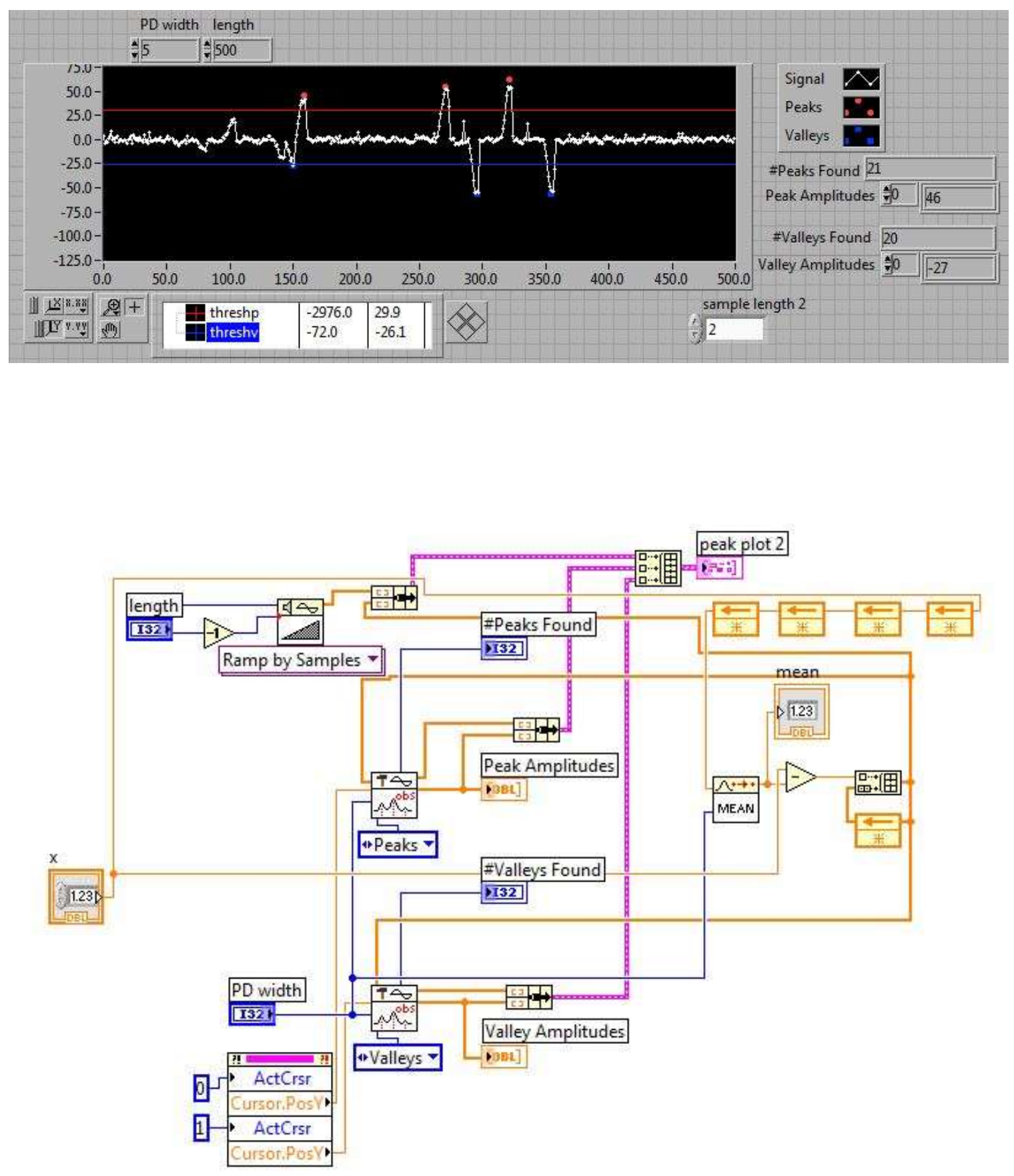

Figure 25 Developed Module to detect the peaks and valleys with their values 
Figure 26 is an example of event detection with this module. In the bottom of the figure are the active and reactive power signals. The lower portion of the figure depicts some devices being turned on and off; the upper portion of the figure is the output of the LabView module explained above.

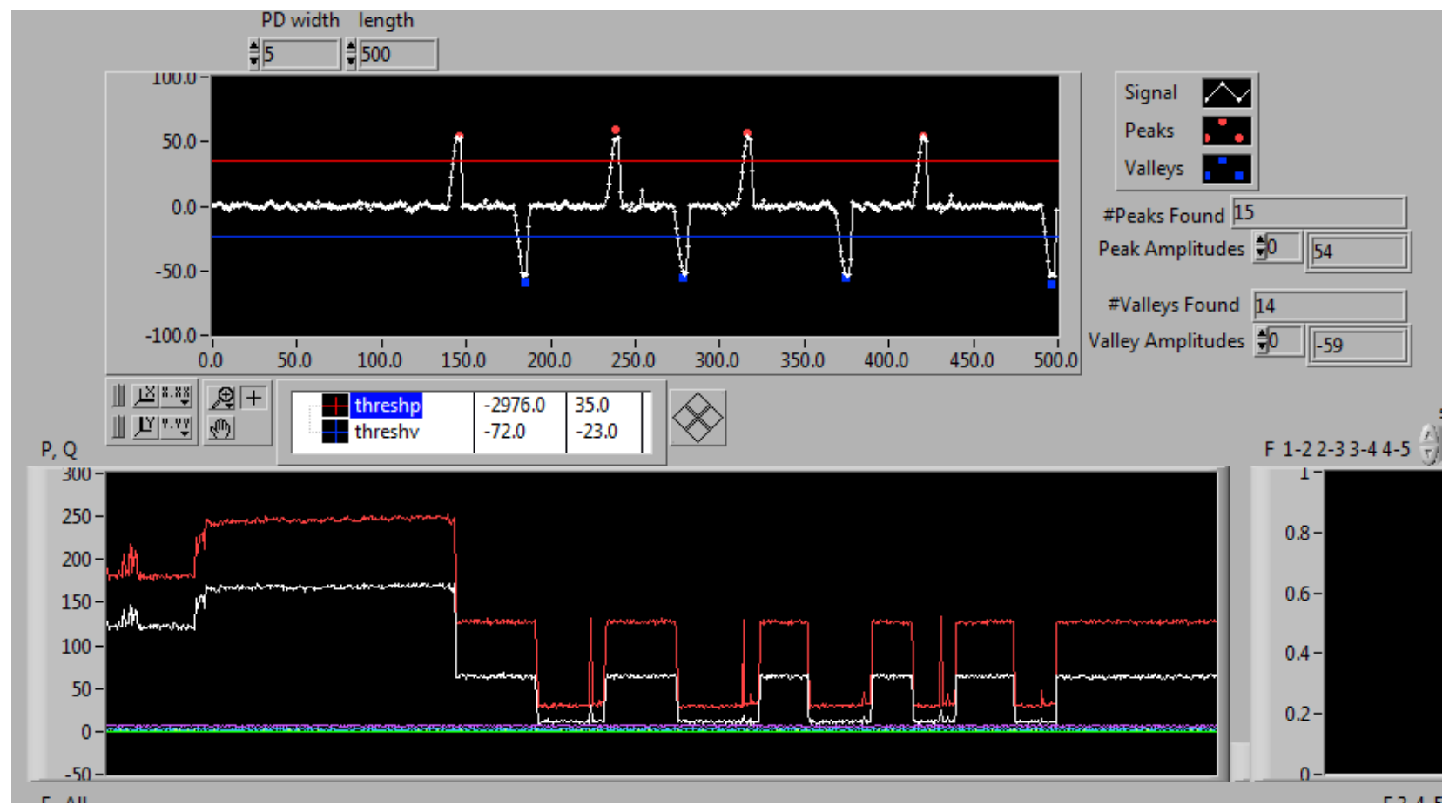

Figure 26 An Example of Real-time detection with developed module in LabView

This method demonstrated some results, but ultimate was not successful due to some problems. As previously mentioned, a major problem that we faced was the lack of computing power; the LabView laptop is not able to handle a lot of calculations. We also encountered problems maintaining a sufficient sampling rate on the laptop when LabView was performing calculations. Because of the problems encountered, it was decided to do the all analysis and calculations with Matlab on a separate workstation, and not in the laptop 
that is used for data acquisition. All of the data are transferred via Internet to the processing computer. The method by which we implemented this was to set and synchronize the output folder of the DAQ system in LabView to a DropBox.com account. In that way, when the data is completed and produced in the DAQ laptop, it automatically will be synced between the two computers without needing (manual) remote access to the DAQ laptop.

\subsection{Event Detection in Matlab}

In the default operating mode, the system samples the power signal at about $12 \mathrm{~Hz}$, so the two second data windows are represented by two 24 index arrays - one containing real power and one containing reactive power. The 24 index power array is passed to a detection algorithm that determines where rapid power changes are located in time. This is accomplished by passing the array through a change-of-mean filter that calculates the difference between the original power signal and the output of a low pass filter. The result is a processed signal that only contains rapid power changes. The process is illustrated in Figure 27 and three example of deploying this method on the power signal is depicted in Figure 28. 


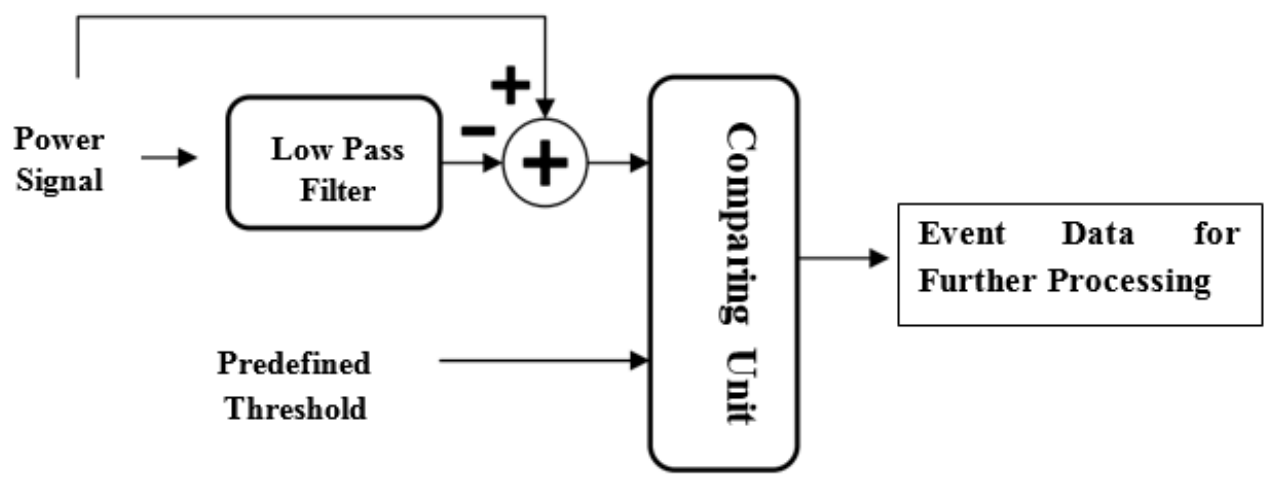

Figure 27 Event Finder Diagram

The filtered signal is then passed to a comparator that identifies any array index where the signal exceeds a preset detection threshold. Three 24 index arrays are initialized to further process the signal: Time, Active Power and Reactive Power. The threshold is set depending upon the environment. For instance, if this system is installed for an industry, then the threshold would be higher than if the system installed for residential use (due to high power consumption loads and nature of industrial electrical demands).

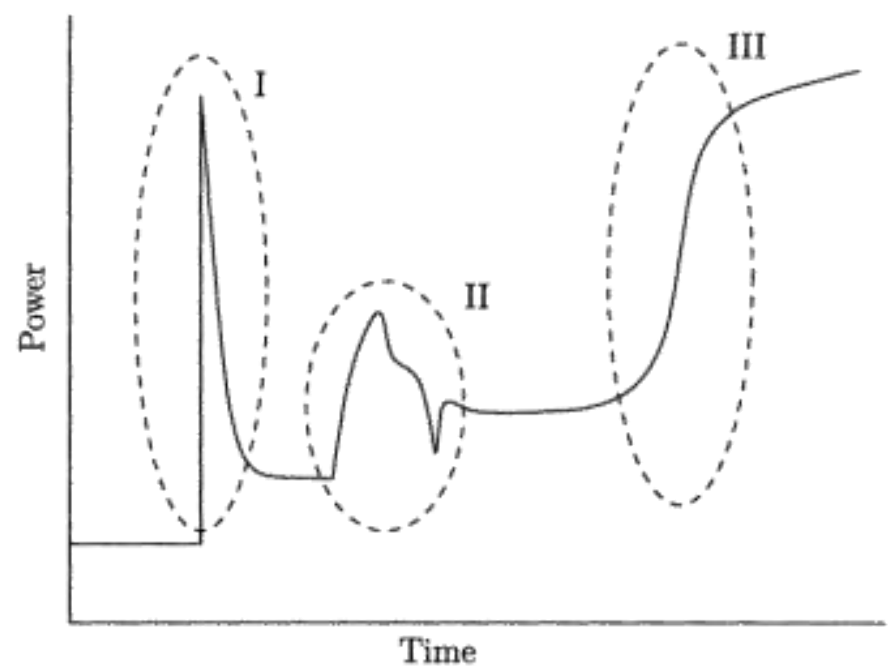

Figure 28 Start-up transient example of three different device 


\subsection{Features Extraction}

In signal processing, feature extraction is a special form of dimensionality reduction. In our case, since the input data to the algorithm is too large to be processed and it is suspected to be notoriously redundant, the input data will be transformed into a reduced representative set of features (or feature vector). Transforming the input data into the set of features is called feature extraction. If the features extracted are carefully chosen, it is expected that the feature set will contain the relevant information from the input data, allowing the detection task to use this reduced representation instead of the full size input. The other important aspect of doing feature extraction is for a later stage of the code; since we are trying to find the best match for each signature, it is necessary to capture features such that similar events have feature vectors that are near each other in vector space. By using feature extraction, we can classify the signatures by their characteristics. Shape matching is not enough to detect the events because some of the events could have the same shape but different values or even different duration. Therefore, there is a need to have a valid procedure to first compare some of the main features of the signals with the prerecorded signals, which can then allow a subsequent step for shape matching.

Feature extraction involves simplifying the amount of resources required to describe a large set of data accurately. In this case, the features are in a matrix along with the data for the shape of the events. After the features are calculated, they are put into a matrix as explained below. 
The first row of the matrix is the device type which is determined by the average power consumption at the transient after passing overshoot or oscillations. The second row is the overshoot time; this represents how much time it takes to reach to the maximum or peak - in some cases it can be overshoot, and in the other cases if there is no overshoot it can be the time that it reaches the nominal power. The overshoot time helps us to find and detect appliance status easier. Even so, since every device has its own power signature at turn-on and turn-off, it is still necessary to make use of other features. The third row is the average power consumption of the device at the transient after passing overshoot or oscillations. The fourth row is amount of the peak or overshoot value of the signal at the transient. The last row is the "area under the curve" (AUC). This feature is selected because in some cases there are some oscillations in the signature at the time the device goes on. The AUC represents the amount of energy (e.g. KWh) used within a given time interval and help to separate different devices. All features are illustrated in Figure 29 and Table 1 shown an example of extracting features along with the signal data for the events shown in Figure 30. 


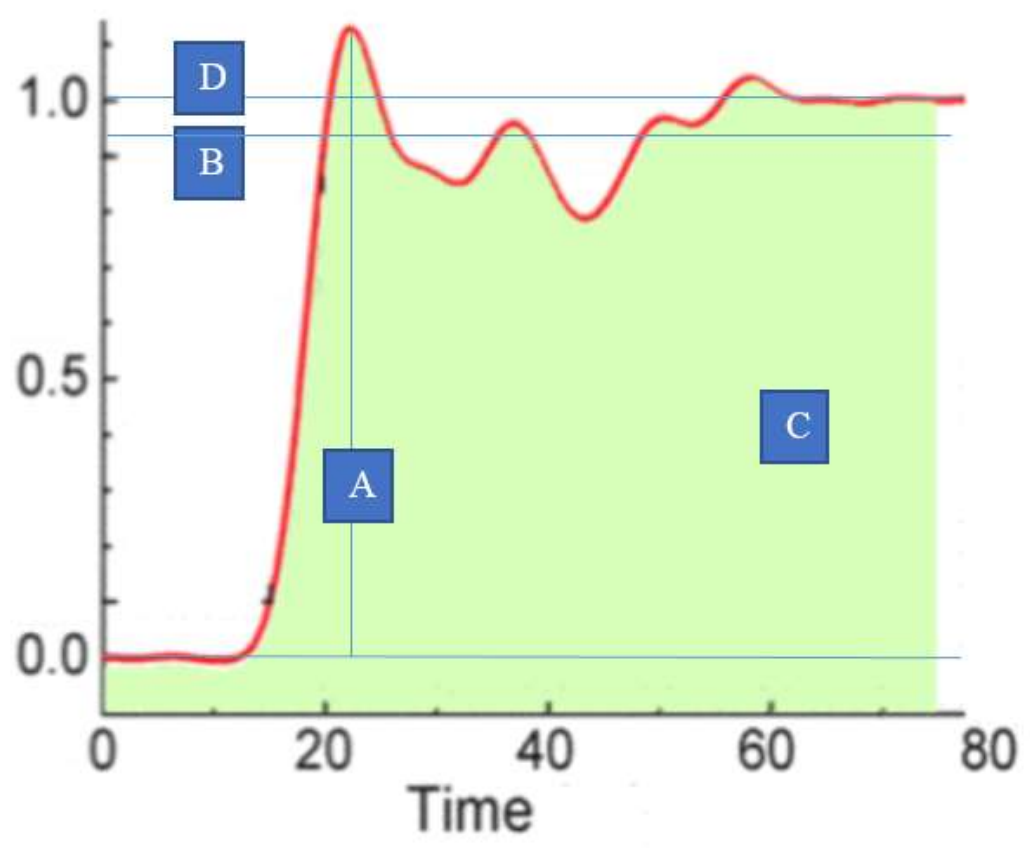

Figure 29 Features used to detect signals a. Overshoot, b. signal average, c. AUC (Area under Curve), d. final value

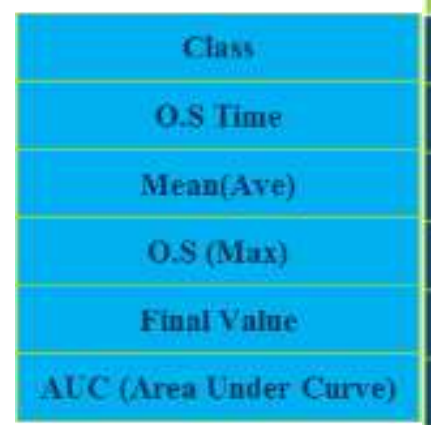

\begin{tabular}{|c|c|c|c|}
\hline $\begin{array}{c}\text { Active Power } \\
\text { Features }\end{array}$ & $\begin{array}{c}\text { Reactive Power } \\
\text { Features }\end{array}$ & $\begin{array}{c}\text { Active Power } \\
\text { Signal }\end{array}$ & $\begin{array}{c}\text { Reactive Power } \\
\text { Signal }\end{array}$ \\
\hline 2 & 2 & 1.207159 & 3.479331 \\
\hline 134.008 & 163.01 & 0.869018 & 0.743569 \\
\hline 255,08 & 52.35 & 415.2768 & 105.1903 \\
\hline 415.27 & 106.37 & 405.4617 & 101.0334 \\
\hline 189.1 & 21.2 & 402.9554 & 106.3728 \\
\hline 167.18 & 36.61 & 363.5012 & 75.36213 \\
\hline & (ii) & 303.722 & 56.68046 \\
\hline & ifi & 273.1344 & 55.58607 \\
\hline & (1) & 250.4979 & 38.45884 \\
\hline & ai & 194.6295 & 16.47704 \\
\hline & (1): & 194.6295 & 16.47704 \\
\hline
\end{tabular}




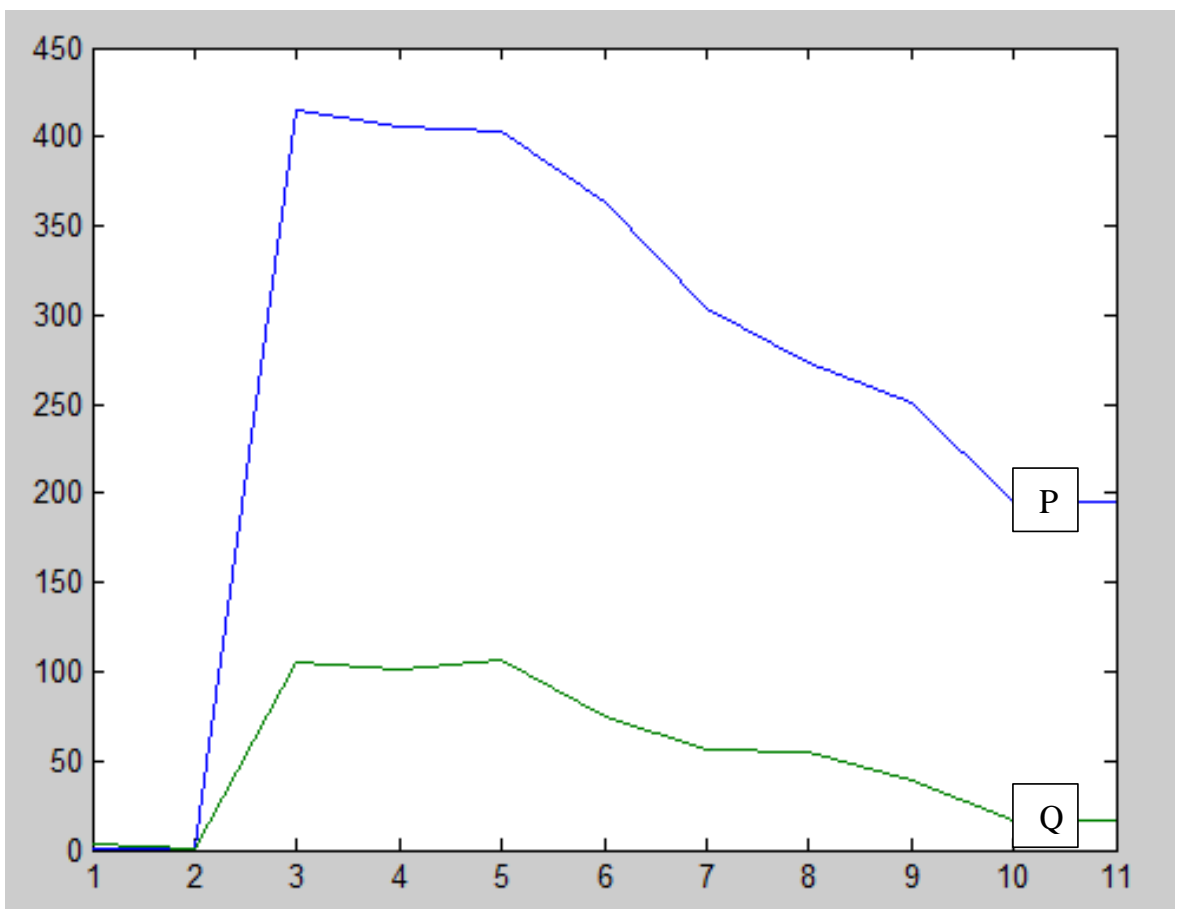

Figure 30 plotted graph of the data from Table 1

When performing analysis of complex data, one of the major problems stems from the number of variables involved. Analysis with a large number of variables generally requires a large amount of memory and computation power. Additionally, a classification algorithm which over-fits the training sample may generalize poorly to new samples. Feature extraction is a general term for methods of constructing combinations of the variables to get around these problems while still describing the data with sufficient accuracy.

\subsection{Reselecting the Features}


In the previous section we tried to use overshoot time, Max Overshoot of the signal, signal average (mean), the amount of difference between the overshoot the average and the final value, and the AUC. A problem encountered is that since the sampling rate is not very high, the rise time (time of Max Overshoot) is not accurate and then this value can falsely indicate differences between two same signals. In Figure 31, the two events shown are the same (from the same device) but there is a difference in overshoot time in the active power. Since we used milliseconds as the time scale for overshoot time in order to have the maximum accuracy, the observed time lags makes this feature not accurate enough to be used as a discriminating feature to detect between the signals.

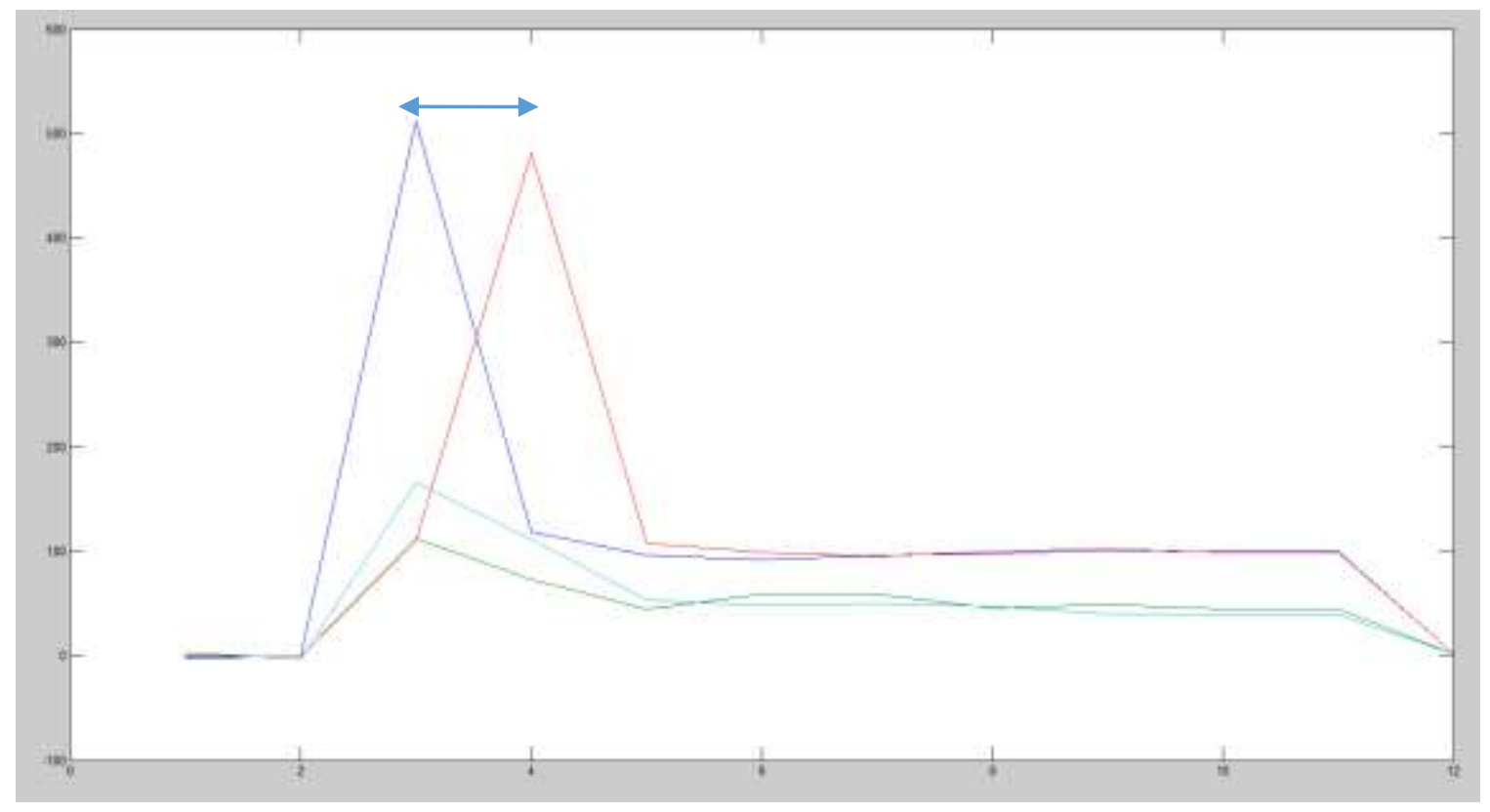

Figure 31 Overshoot time difference between the two same power signatures for the same device

In order to solve this problem the set of features used for classification should be changed. The new features selected include Max Overshoot of the signal, signal average (mean), 
Minimum of the signal, the average of two last samples which is kind of the last value of the signal in the windows of looking and the last one is the AUC( area under curve).

With these features there is some improvement, but there are still some problems. Minimum of the signal in the large loads is a great feature, but for the low consumption loads when some of the large loads are already online (making electrical noise) and then the power signal has fluctuations, it makes it hard to find the right minimum for small loads. It gets worse for the reactive power signal when there is a large load online and the device that we are trying to detect does not have reactive power at all (or it is very small). In the Figure 32 the power signatures are depicted for a lamp (40 Watt). The left one is the turning $\mathrm{ON}$ signature, and it is clear the active power jumps and goes up to about $40 \mathrm{~W}$; however, the reactive power decreases and fluctuates which is not correct for that device. The orange lines give the power signature representation of the lamp without having any other loads online. In the right, it is the same scenario for the lamp when it goes OFF; the reactive power signature in this situation is not that useful.

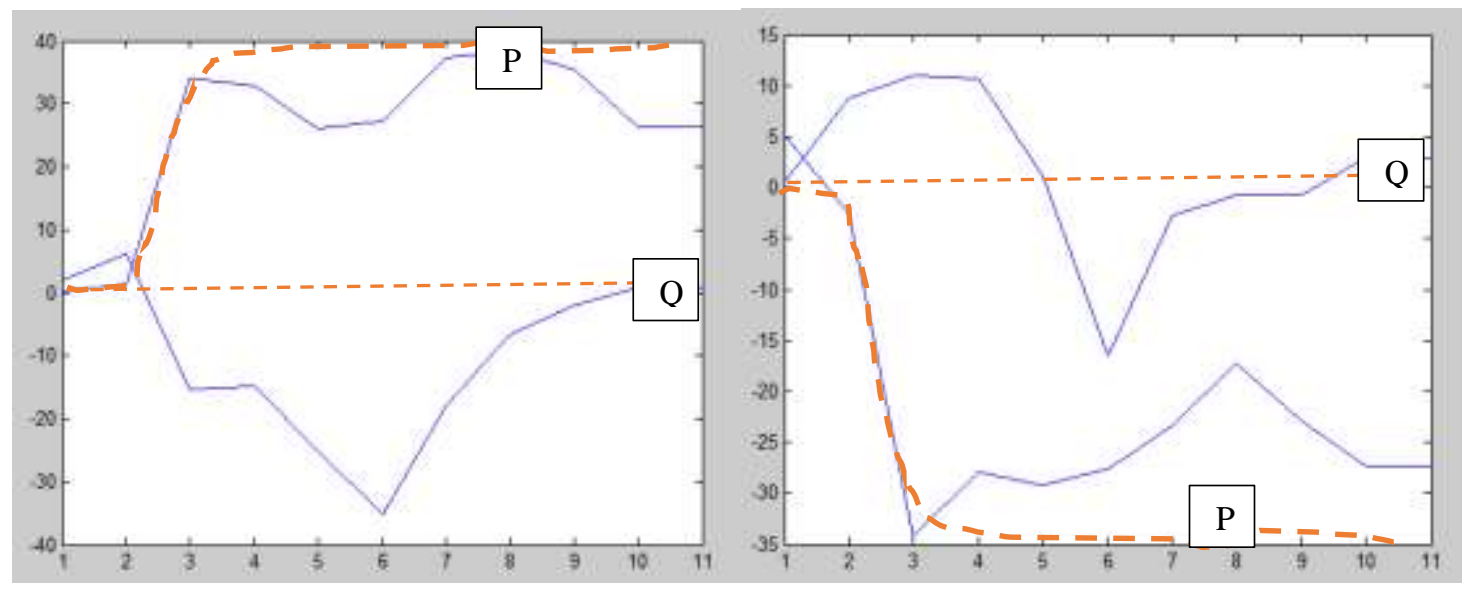

Figure 32 Low Consumption loads issues 
In order to overcome this problem a bit, in class zero (which is assigned for devices under $100 \mathrm{~W}$ ), the minimum feature mentioned above is not being used to find matches. Additionally, the reactive power features are not used for classification of devices in class zero.

Figure 33 illustrates an overview of the Feature matching and also next section regarding the shape matching. 


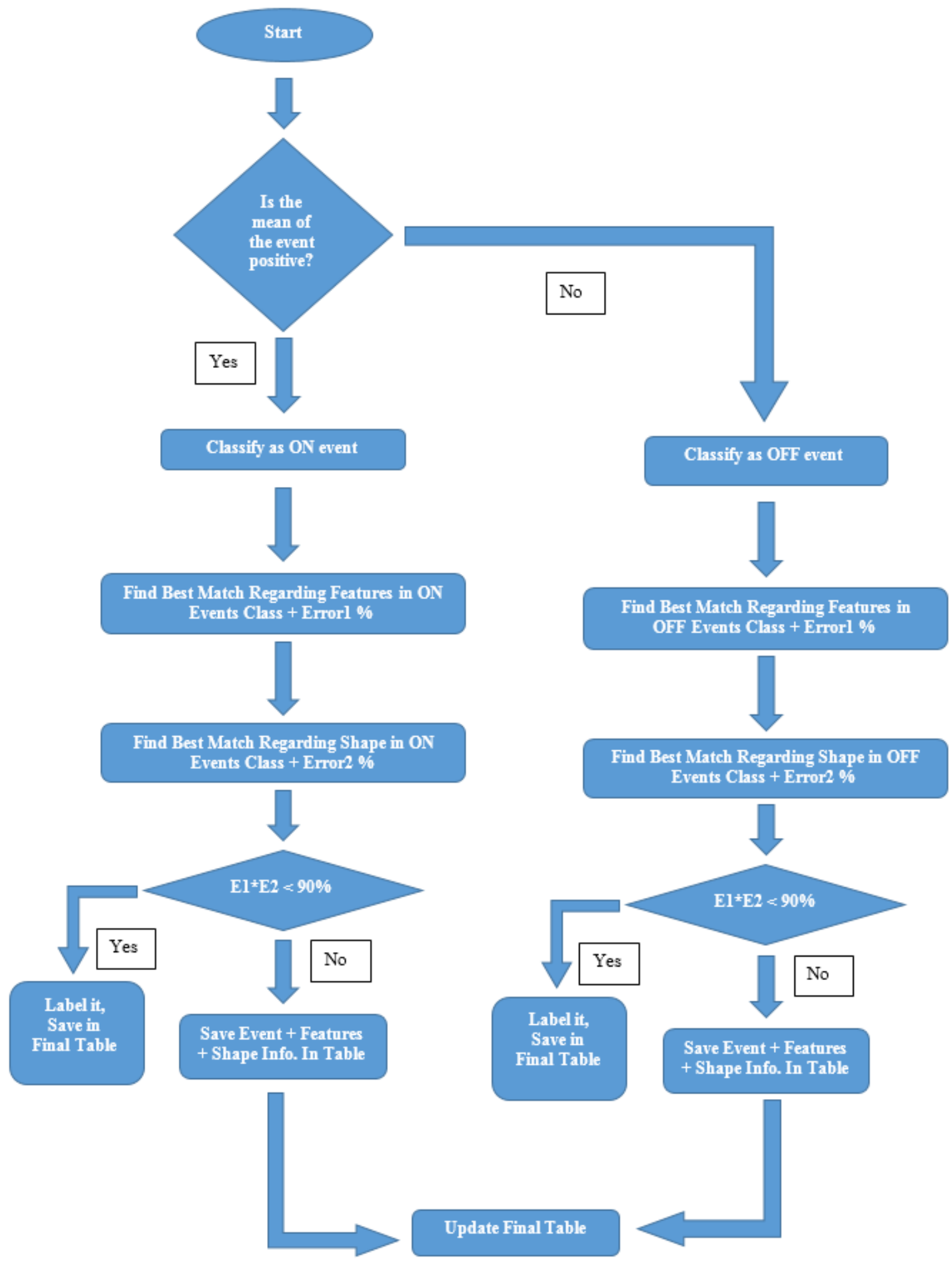

Figure 33 Matlab algorithm for signal classification 


\subsection{Signature Shape Matching}

After the code tries to find the best and nearest matches using the feature matrix, and then if the differences are reasonable, it will go and try to find the best matches using the signal shape and format by using the cross-correlation (xcorr) function in Matlab. The reason that the code is not trying to find the status at the beginning by shape comparison is that the cross-correlation function looks only at the shape and not the amplitude (or the other extracted features). Thus, relying solely on cross-correlation would likely give incorrect classifications when we can effectively use other features with more discriminating power. We need a way to filter and classify the signals first by using a feature extraction function. Subsequently, since some of the events could have a similar features, the code then tries to use cross-correlation to find the best match. Additionally, cross-correlation will shift the signals to find the best match positions, so if we use this function directly without any other method to validate the signals, we will get poor classification because some signals look the same when they shifted a bit.

One example of the cross-correlation function is described below. In Figure 34 a specific event from the prerecorded data is depicted. In Figure 35, the same event happened and after feature matching we must perform shape matching. This shape matching (crosscorrelation) will find the correlation ratio between this unknown (unclassified) event and events that are already classified. (The process of event classification will be described in Section 3.6.) As shown in Figure 36, the output of the cross-correlation will yield a peak 
at the point where the shape of the signal is most similar, and the value of the crosscorrelation at this peak (which we will call $x$ corr $_{\max }$ ) is used to determine the similarity between the compared events. For instance, in this case $x c o r r_{\max }$ equals 0.9976 (where 1.0 would represent exactly identical signals). The threshold for $x$ corr $_{\max }$ that we used in this project for the accuracy is about 0.85 . Therefore, after finding the pre-classified event with the nearest feature vector, if $x$ corr $_{\max }=0.90$, then the unknown signal will be newly classified as the same event. If the $\operatorname{xcorr}_{\max }<0.85$, then it means that we have detected a new device (because no such event currently exists in the table of pre-classified events). Therefore, this new device (event) is recorded in the table so that similar events can be recognized in the future.

$\max \left(x \operatorname{corr}\left(\right.\right.$ event, table events, ${ }^{\prime}$ coeff' $\left.)\right) * \max \left(x \operatorname{corr}(\right.$ event, table events $),{ }^{\prime}$ coeff' $\left.)\right)=.9976$ 


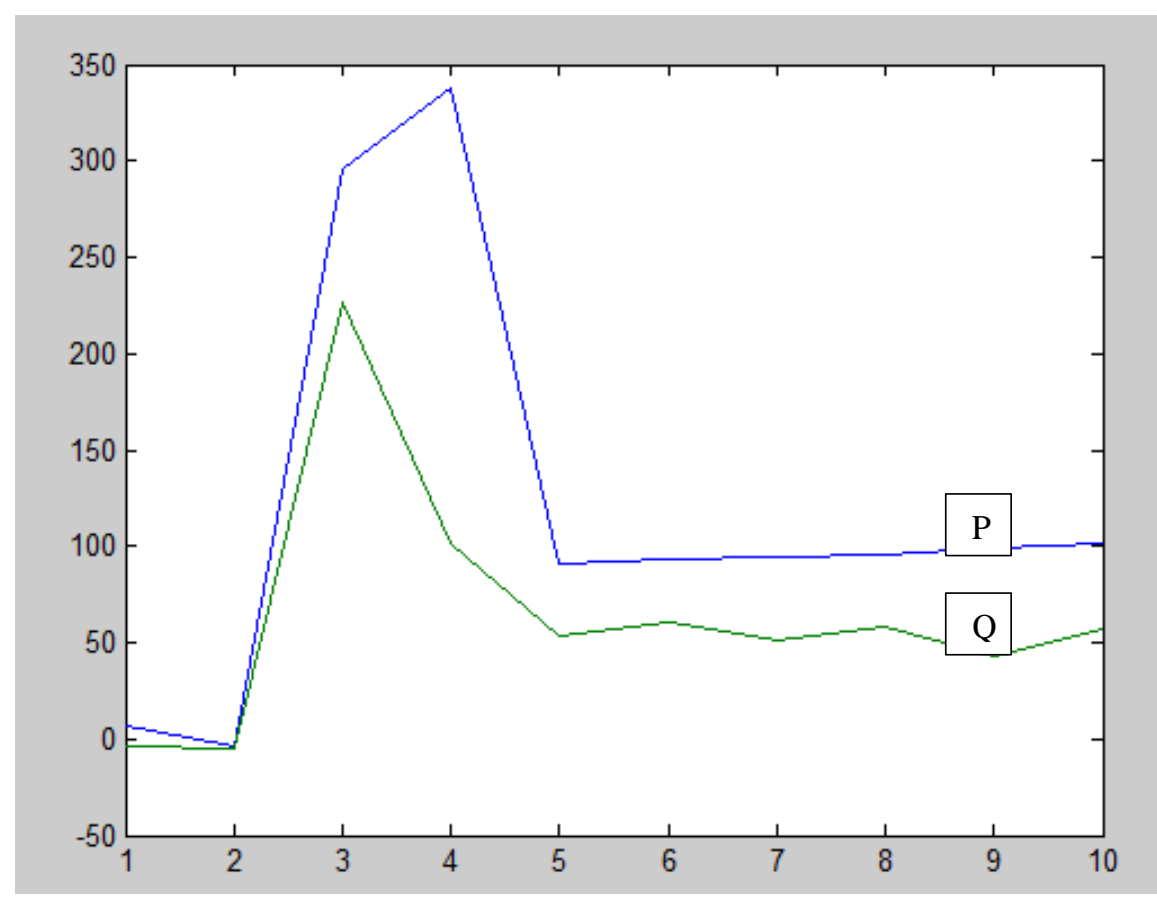

Figure 34 Signal Shape representation of a specific event

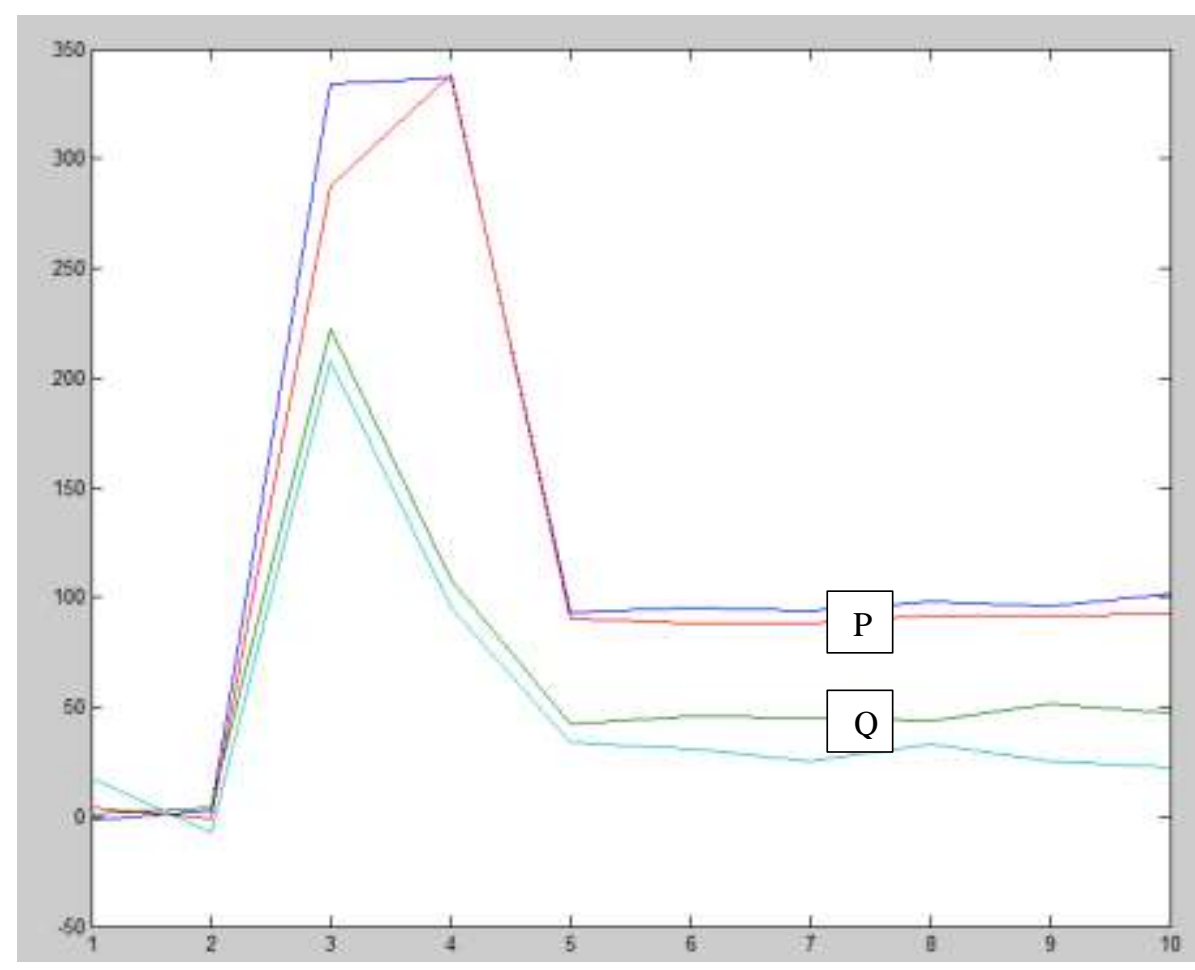

Figure 35 Two events with the same signature 


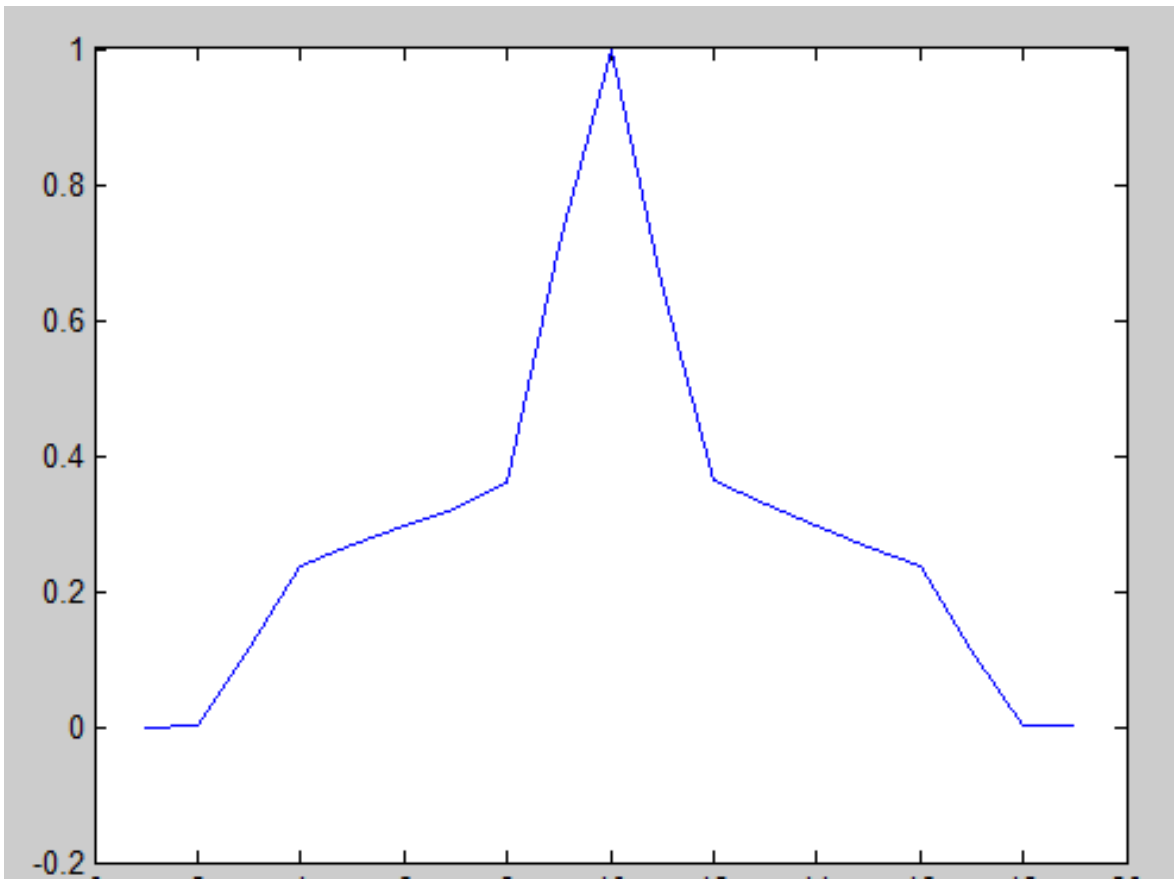

Figure 36 Cross Correlation output for the Figure 35

\subsection{Automated Event Detection/Classification}

In order to find the best match (if one exists) for each unknown event, the system automatically detects the signatures and compares it with the table of event feature matrices that are already classified from prior observations. If no matches are detected, the system will add the feature matrix of the unknown event into the table as a "newly discovered" device. Additionally, the signal data is saved so that the cross-correlation with future "unknown" events may be calculated for future classification.

Table 2 represents configuration of the mentioned table and the data is for the event shown in Figure 37. 
Table 2 Sample of a Recorded data in table

\begin{tabular}{|c|c|c|c|}
\hline $\begin{array}{c}\text { Active Power } \\
\text { Features }\end{array}$ & $\begin{array}{c}\text { Reactive Power } \\
\text { Features }\end{array}$ & $\begin{array}{c}\text { Active Power } \\
\text { Signal }\end{array}$ & $\begin{array}{c}\text { Reactive Power } \\
\text { Signal }\end{array}$ \\
\hline 10 & 10 & 7.246445 & $-\mathbf{2 . 8 6 8 7 2}$ \\
\hline 201.012 & 134.008 & -3.40095 & -1.78428 \\
\hline 1067.817 & 170.9636 & 754.7667 & 624.0476 \\
\hline 1389.643 & 624.0476 & 1389.643 & 159.9359 \\
\hline 321.8259 & 453.084 & 1382.42 & 160.0557 \\
\hline 644.0641 & 109.6266 & 1376.162 & 157.3865 \\
\hline 0 & 0 & 1372.856 & 157.1883 \\
\hline 0 & 0 & 1369.14 & 153.5242 \\
\hline 0 & 0 & 1366.743 & 157.8362 \\
\hline 0 & 0 & 1365.204 & 157.6391 \\
\hline 0 & 0 & 1365.204 & 157.6391 \\
\hline
\end{tabular}

The first column contains the features for the active power signal, second contains the features of the reactive power signal, the third contains the signal data and shape of the active power, and the last column contains the signal data and shape of the reactive power.

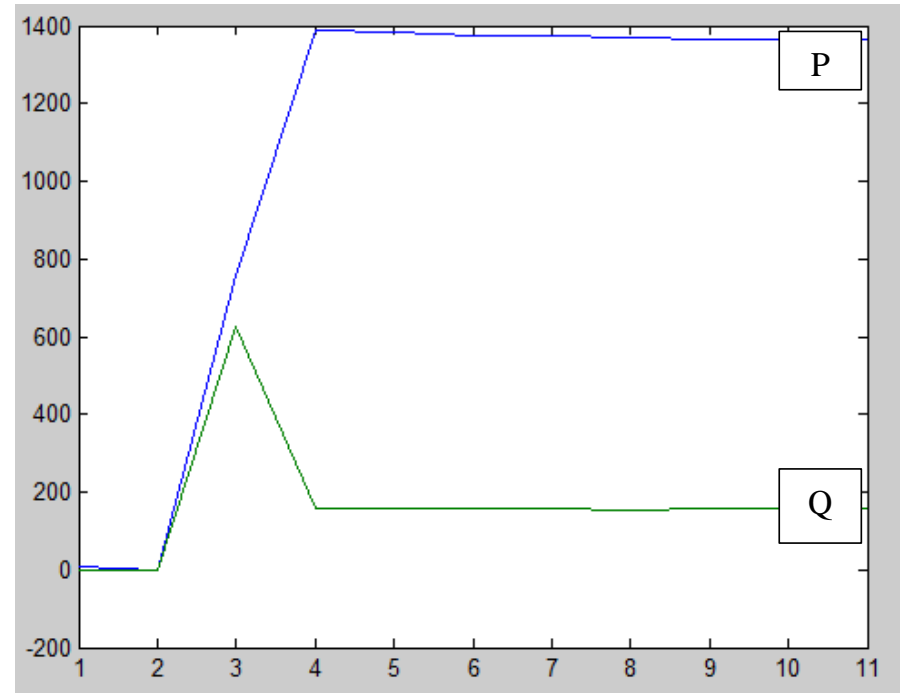

Figure 37 Representation of Active and Reactive Power Signals of Table above.

The software for event detection consists of 5 different sections. The first part is to import the date from the text file to Matlab. By running the code, a window will pop-up and ask 
for the data file (which is typically a text file). Since there are different ways to save data in the text file, in the next step the code tries to determine the column numbers for the active and reactive power of each phase as well as the time column to make sure that it will extract all information correctly. After extracting the data from the text file and putting it in a matrix, the system will detect the number of phases. If the system is single phase it will leave the data as-is, but if the system has two or three phases the code will accumulate the phases to construct only one active power and one reactive power signal. Processing one active power and one reactive power signal is much easier than processing 4 or 6 signals. The advantage of this approach is that even though some appliances use two or three phase, by processing the accumulated power signal it is easier to detect the signature of every device by a common method.

The second part of the classification software finds and detects the reasonable changes in the power signal. This calculation requires a defined threshold to detect only valid events and ignore the noise and other fluctuation in signals. After considering the power signal and experimenting with some values, a minimum change of 35 watts was determined to be a good threshold (which means that the code is not able to detect loads below 35 watts. For instance, if there is a load such as a small lamp (10 Watts), if it goes ON or OFF it is not possible for the system to detect that and it will be treated as noise or some irrelevant changes in the power signal. The reason that the threshold should be 35 Watts and not less is because setting this value lower (5 Watts for example) will result in a huge number of events, many of which are likely just noise or changing in the loads.

Detecting the events can be accomplished simply by defining a sliding window on the power signal and calculating the signal average for at least 11 samples in a row (which is 
the same size as window that is defined to roll over the signal). The sliding average window is like a low pass filter that smooths the rapid changes which are like higher frequencies in the signal. By comparing the average value point-by-point inside the window against a specified threshold, if a value is found that exceeds the threshold it will detect it as an event (see Figure 38).

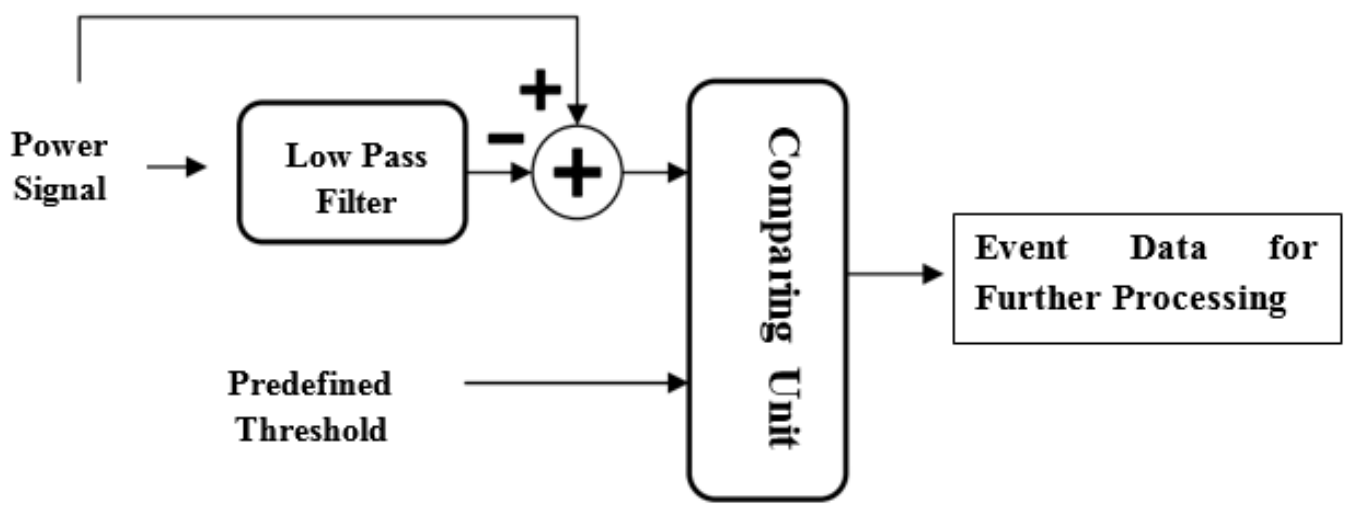

Figure 38 Event Detection diagram

By considering a small "window" of the signal, these algorithms look for evidence of a step occurring within the window. The window slides across the time series, one time step at a time. The criteria for detecting a step were determined experimentally.

The third part of the code is for finding the valid events while cancelling-out the others. Some events that are detected by the event detection section may not be actual events (for instance, it could be large noise, LabView lag issues, laptop RAM issues, or some other 
problems mentioned earlier). "Noise events" may occur where there is a very short spike in the power signal lasting less than 100 milliseconds. The process measures the duration of a candidate event from the signal, and if the duration is too short the candidate is consider noise and is deleted. For valid events, it was determined experimentally that capturing 0.5 seconds of the event provided sufficient data for extracting features and subsequent classification. Figure 39 depicts one event for 0.5 seconds. The other part of the code in this section is used to prepare the signal and normalize it such that the beginning of the captured event starts from a "zero" level (see Figure 39, lower portion). Normalizing the event signature in this manner allows for consistent feature extraction and crosscorrelation, as described earlier in this chapter. To determine the baseline value (offset) to subtract to accomplish this normalization, the signal immediately before a detected event is averaged over a short window (about 11 samples total). This offset is then subtracted from the signal over the 0.5 seconds of interest to produce the event signature for classification. 


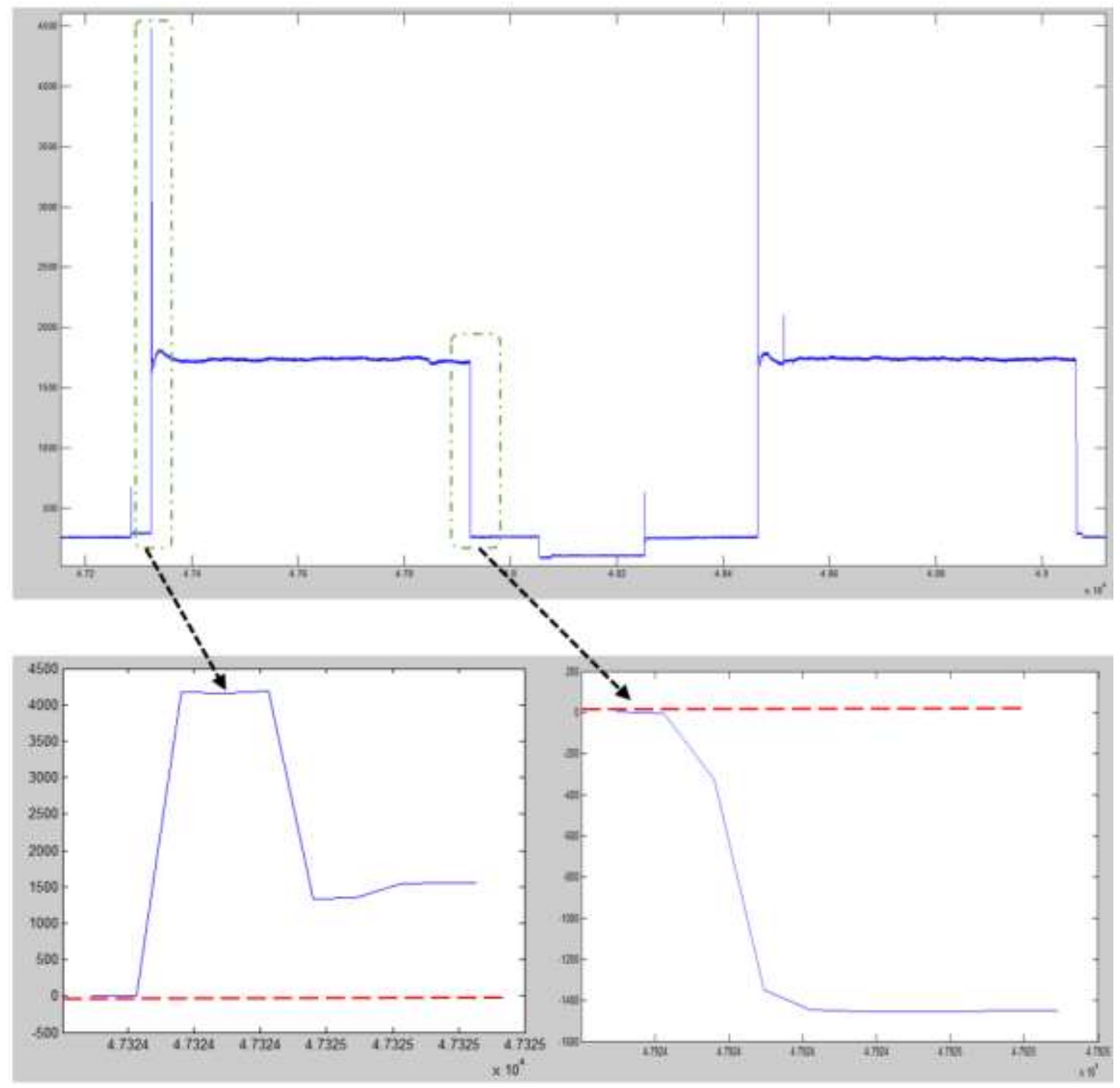

Figure 39 Turning ON-OFF power signal (up), cut and trimmed turning ON power signal signature (down-right) cut and trimmed turning OFF power signal signature (down-left)

Another element at this stage is resampling the data for each event to make all of them have the same sampling rate. Different sampling rates are caused by LabView software and Windows issues (RAM leakage, paging files, etc.) as described in section 2.3.2. 
The fourth part of the code is to find best match for each event through the table of data that has already been fed into the code. First, for each event the code tries to find the best match based upon the features that are extracted from the signatures and signals. The method finds the nearest distance between the event feature vector and all the prerecorded feature vectors. In order to do that, we use simple subtraction between the elements of the feature vectors and then add all the distances together to find the total distance. The nearest one is the closest match. Since some events could be new and finding the close match will not help to identify the correct device, another mechanism is needed. If the total distance is less than the average power of the event, then it is acceptable; if not, it will not go through the next level the code, instead saving the feature vector and the shape information (the resampled data of the event) into the table, labeling it as a new device.

As already mentioned, one of the features is the class type. Note that this "class type" does not refer to the classification of similar events, but rather is a function of average power consumption by the device during the $\mathrm{ON}$ or OFF event. For instance, class 0 is for the appliances which consume power between 0 and 100 Watts, class 1 is for the appliances which consume power between 100 and 200 Watts, class 2 is for the appliances consume power between 200 and 300 Watts, and so on. The only issue with defining class type in this manner is that some events will have an average power consumption (during the $\mathrm{ON}$ or OFF event) near the boundary of the classes. Assume a device consumes 202 Watts in a normal condition during the ON event. Since this value is not precise (exactly constant) for each $\mathrm{ON}$ event of this same device (and further may depend on the temperature and a lot of other things), this device can exhibit a bit different power consumption during each ON event. Usually the power consumption during the ON (or OFF) event for each device 
will fall within a range. Assuming the range for the device just described is between 195 Watts to 207 Watts, then assigning the right class type for the event would be a problem. In order to overcome this problem, during event detection, each unknown event is compared with preexisting events within the class to which it is assigned, and also to the events within one class above and one class below to make sure a "borderline" device does not miss the opportunity to be classified with other similar events.

Next step is to try all signals and signatures in the classes that match with the class of the events (including one class above and below) and then by using cross-correlation function find the best match through the table. Initially, we tried to use the correlation function to find the best match, but the problem with that is since the sampling rate is not high and also the start point of the signatures can be a bit shifted in time, the correlation function is not a good choice and gives the wrong results. Therefore, the signal may need to be shifted a bit to find if it fits with one of the signals in the table or not, and the cross-correlation naturally provides this result.

In signal processing, cross-correlation is a measure of similarity of two waveforms as a function of a time-lag applied to one of them. This is also known as a sliding dot product or sliding inner-product. It is commonly used for searching a long-signal for a shorter, known feature. It also has applications in pattern recognition, for discrete functions, $\mathrm{f}$ and $\mathrm{g}$, the cross-correlation is defined as:

$$
(f \star g)[n] \equiv \sum_{m=-\infty}^{\infty} f^{*}[m] g[n+m]
$$


The cross-correlation is similar in nature to the convolution of two functions.

For instance, in the Figure 40 there is one example of this issue. In the active power signals it is very clearly shown that the peak is not always at the same spot, but the whole signal has the same shape and should be classified together.

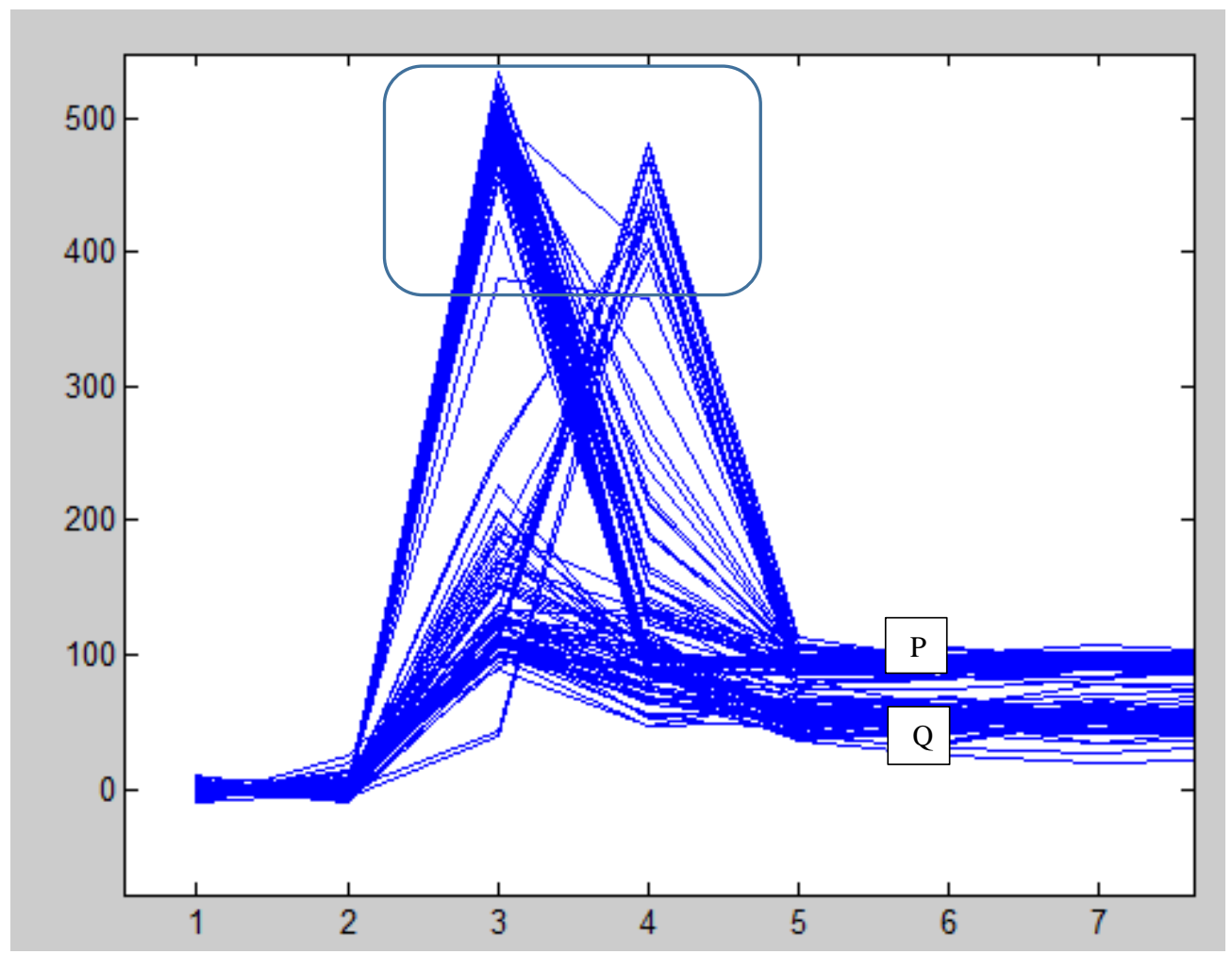

Figure 40 Time shifting issues in the signals

In the following figures there are two examples of the events with similar signatures that went through the feature matching and then shape matching. The features of each signal in Figure $41 \& 43$ are depicted and compared to each other in the Figure $42 \&$ Figure 44. 


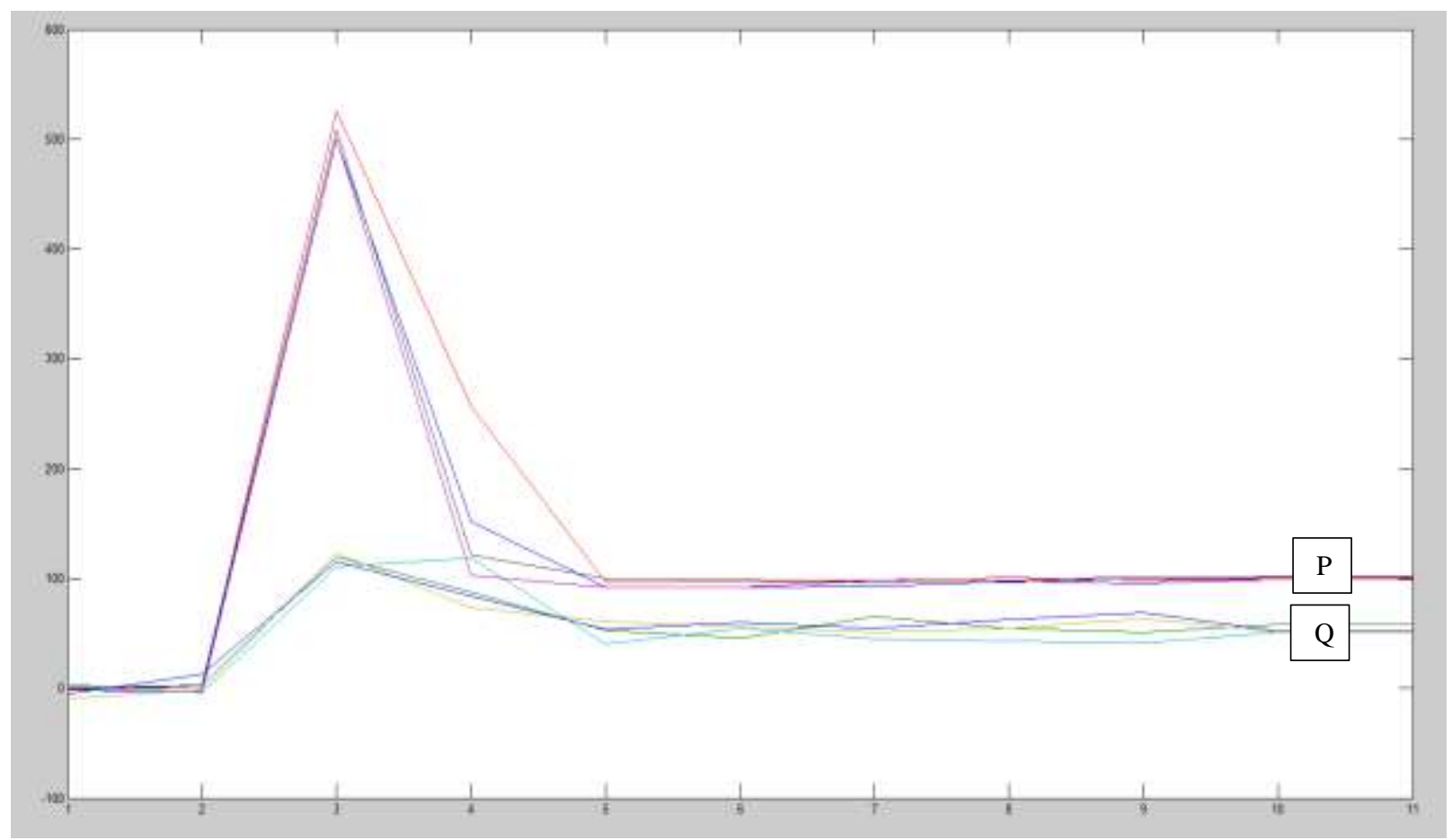

Figure 41 Four turning ON events with the same Signature

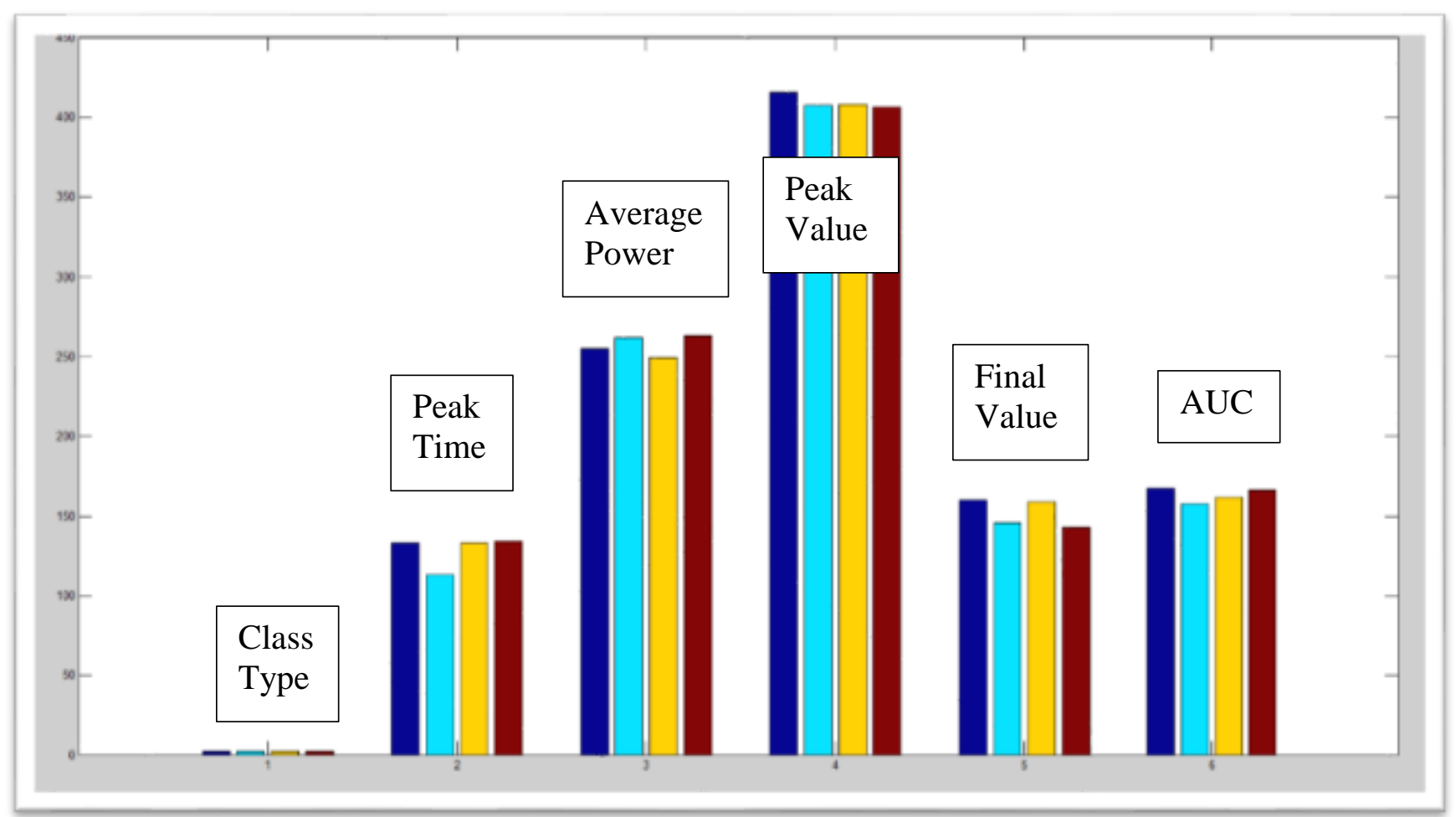

Figure 422 Features of Active Power for four turning ON events shown in Figure 42 


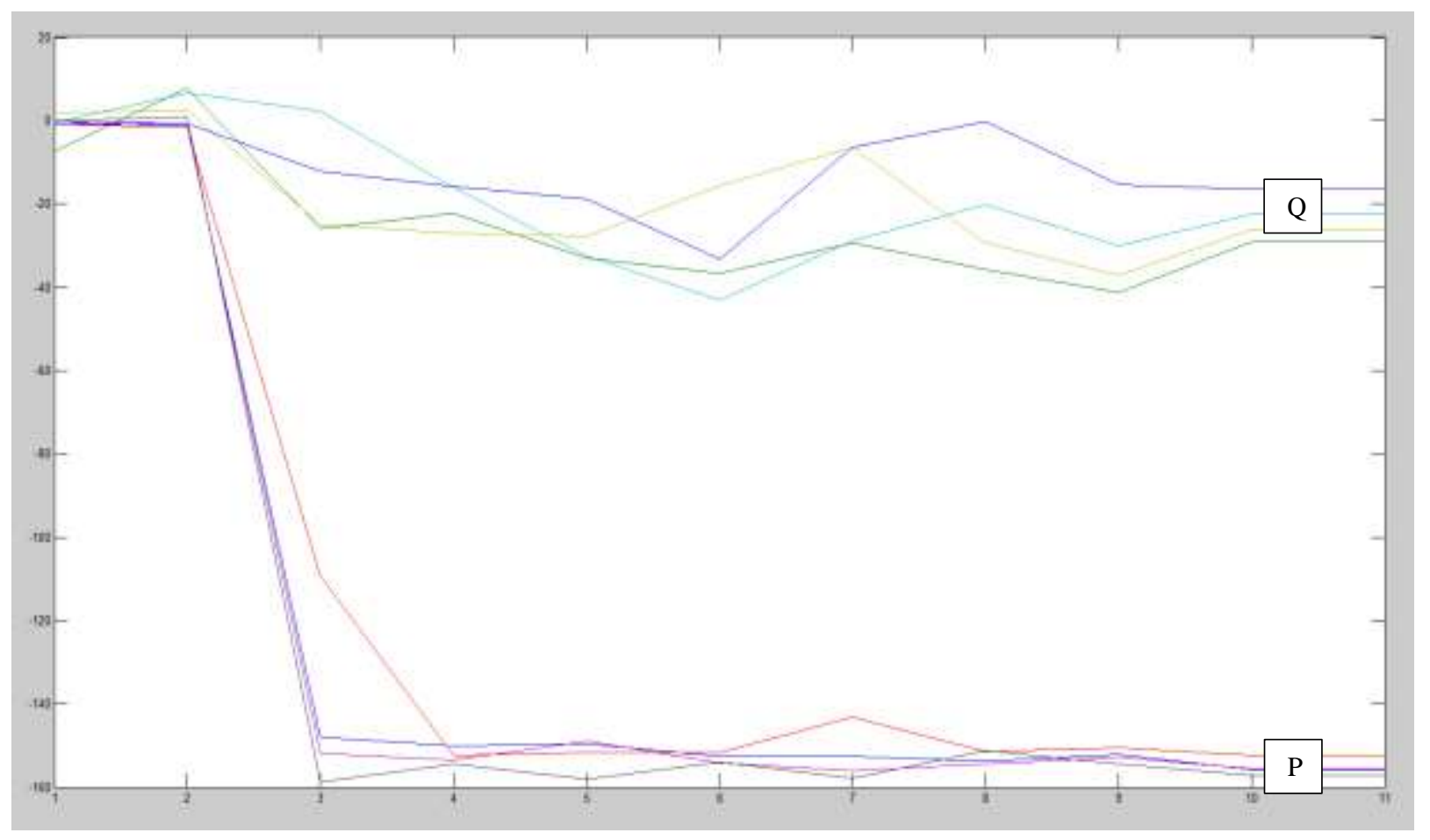

Figure 433 Four turning OFF events with the same Signature

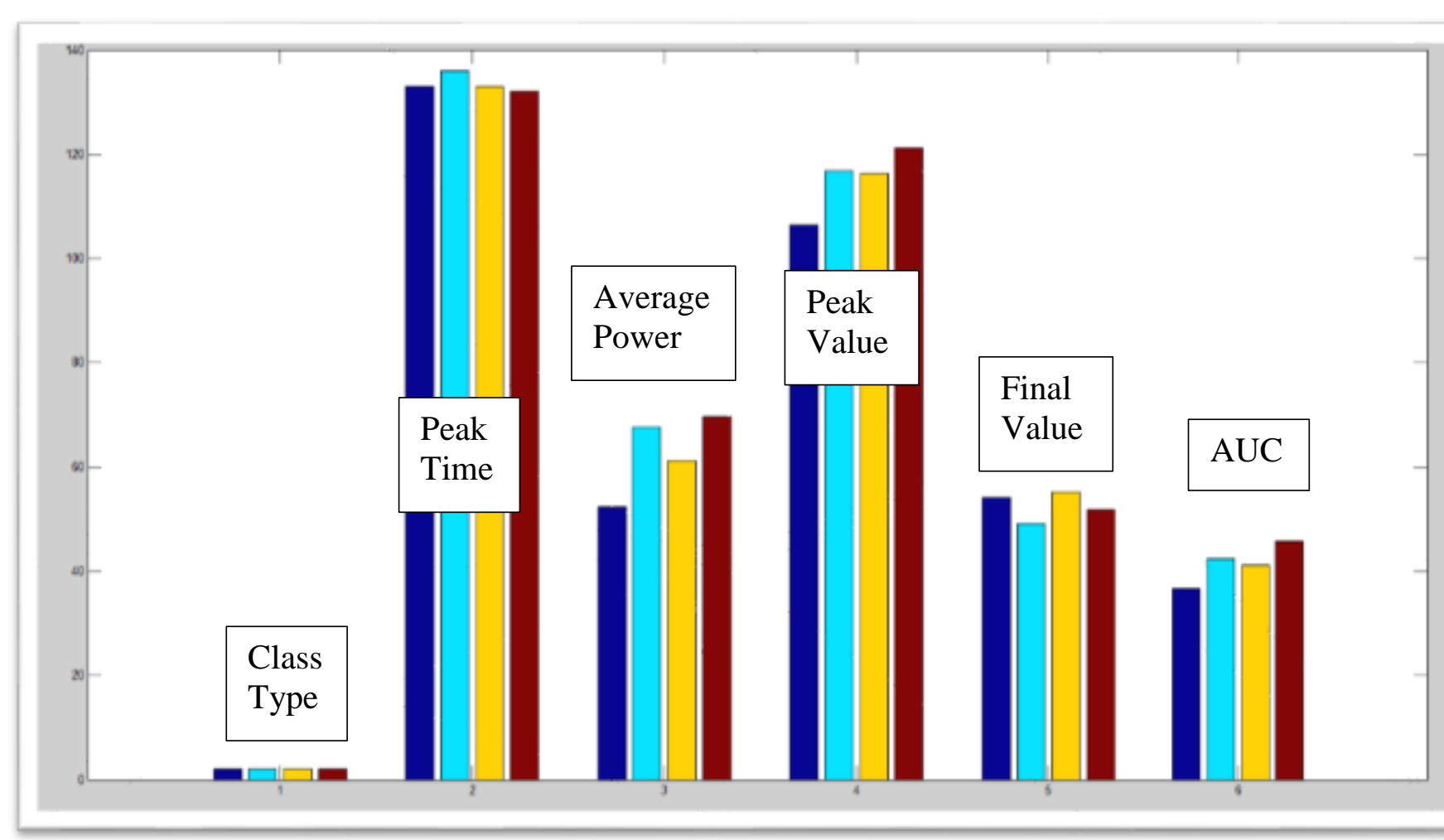

Figure 44 Features of Reactive Power for four turning ON events shown in Figure 42 

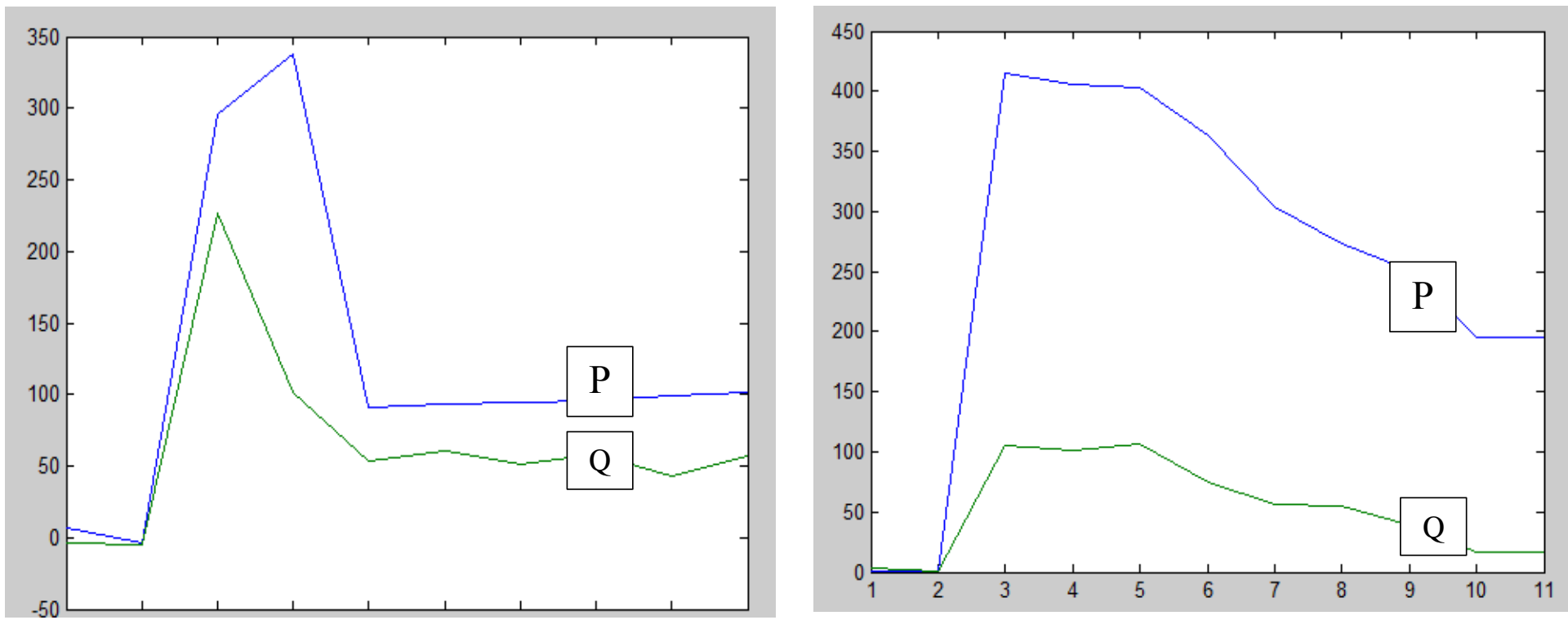

Figure 45 Two different events (active and reactive power) 


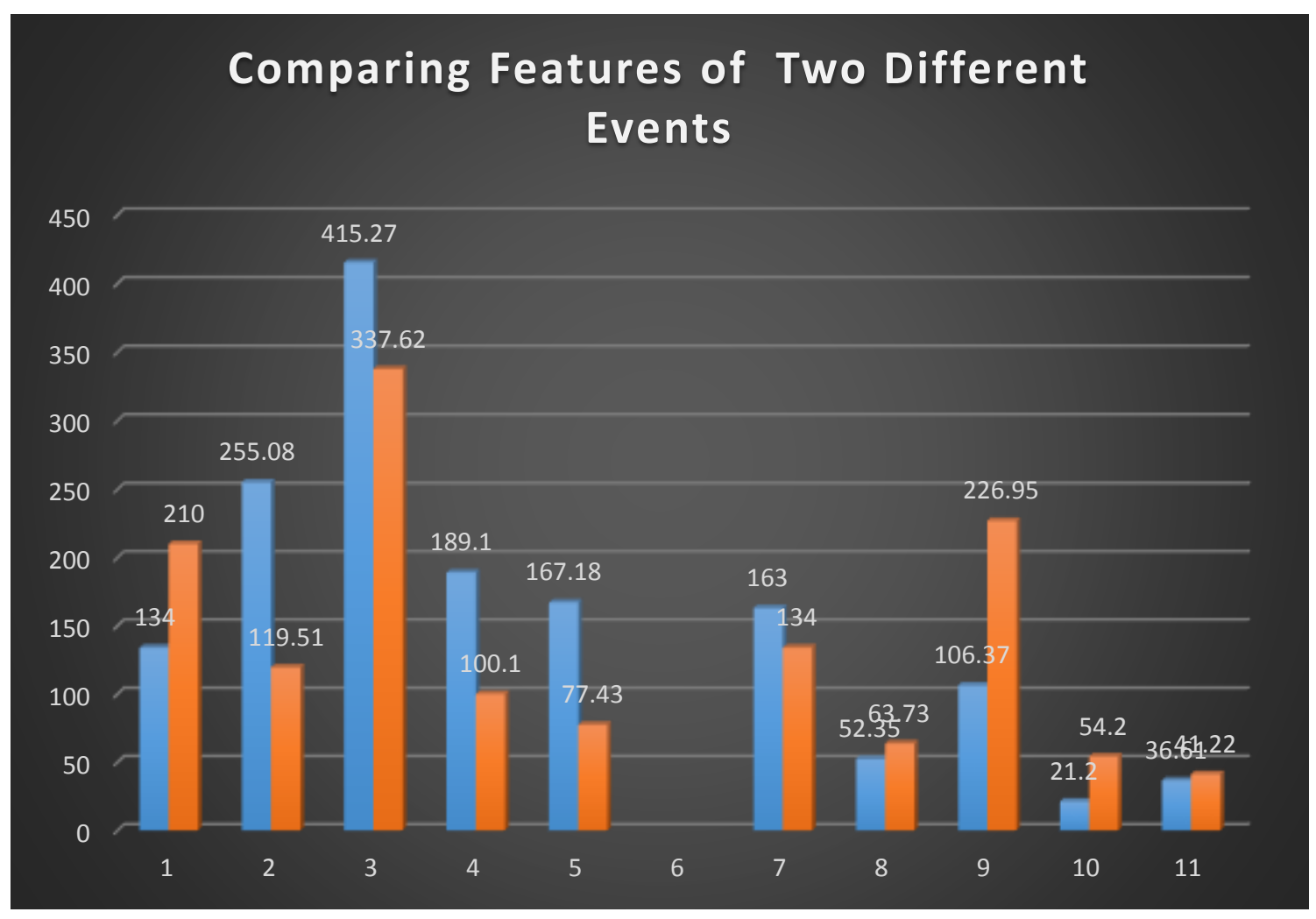

Figure 46 Comparing features of two events shown in Figure 45

Figure 45 showing two different events and Figure 46 illustrates comparing the extracted features from two events shown in Figure 45. This Figure clearly represents that even the events close together regarding the maximum peak and average power, still in the features there are some of them can be used to distinguish between the signals.

If there is no close match detected for a particular event, then the code identifies it as a new device stores all the features to label it as a new device in the table. Since the signal shape is also needed later to compare against other similar events, the signal data (after being prepared and filtered by a low pass filter to cancel the noise) is also stored in the table. 
The fifth part of the code is to count and sort all the same events with the time stamp for each event and then give a report for each device. This part also produces several types of representation of the data.

Figures 47 below are chart representations of the data. Each number on the horizontal axes represents a specific event and the events are ON or OFF only. The vertical axes represent the number of counts for each event. Negative values on the vertical axes indicate OFF events and positive values indicate $\mathrm{ON}$ events. Pie chart in Figure 48 only represents $\mathrm{ON}$ events.

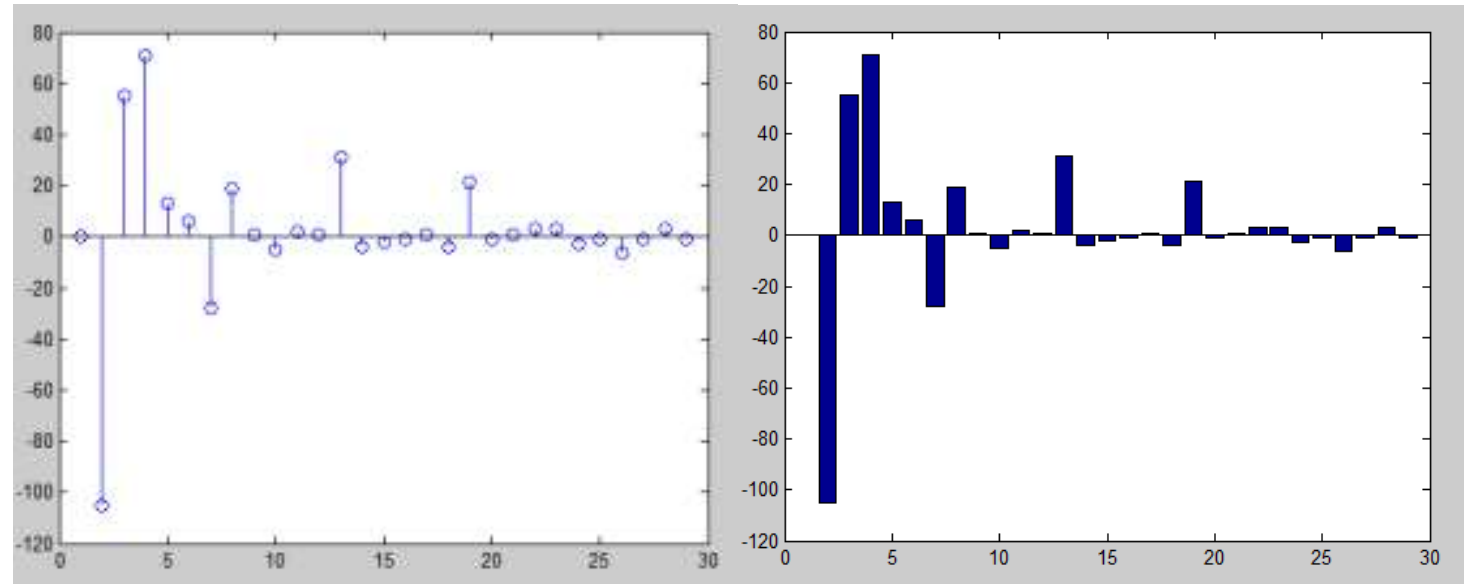

Figure 47 Visual Representation of the number of turning $O N$ and OFF for each appliances 


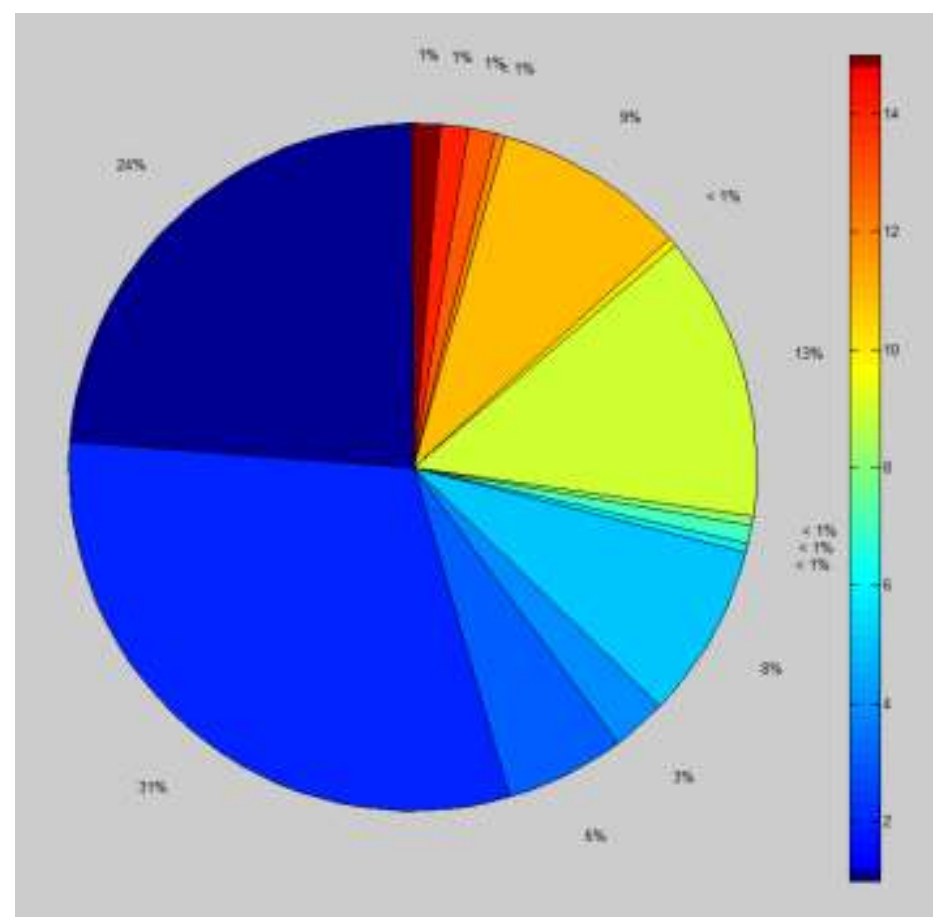

Figure 48 Visual Representation of the percentage of ONLY turning ON for each appliances 


\section{Results and Conclusion}

The low cost Non-Intrusive Load Monitoring installed in TigerPlace shows great promise for future applicability. The single point connections required for a NILM and the capability to monitor the usage pattern of multiple systems in the house are ideal to keep the privacy of residents while making it possible to record the activity that may ultimately detect chronic disease or other issues in the early period of their problem. Early illness recognition and early treatment is not only a key to improving health status with rapid recovery after an exacerbation of a chronic illness or acute illness, but also a key to reducing morbidity and mortality in older adults and controlling costs of health care [2]. In this chapter, some results demonstrating the viability of this task are discussed, along with some observed limitations.

Figure 49 and Figure 50 represent of two days data of a residential home. The largest number of $\mathrm{ON}$ and $\mathrm{OFF}$ events are for the air conditioner inside the house. The pattern of using the other appliance is a bit different from the other day.

In Figure 51, the line pattern of ON/ OFF events for two days is shown and it is clearly depicted that there is a certain pattern of usage devices and appliacnes in the home. In day 2 as it is shown the line is longer and it means that there are 3 new events are detected that were not available at the day 1 . 


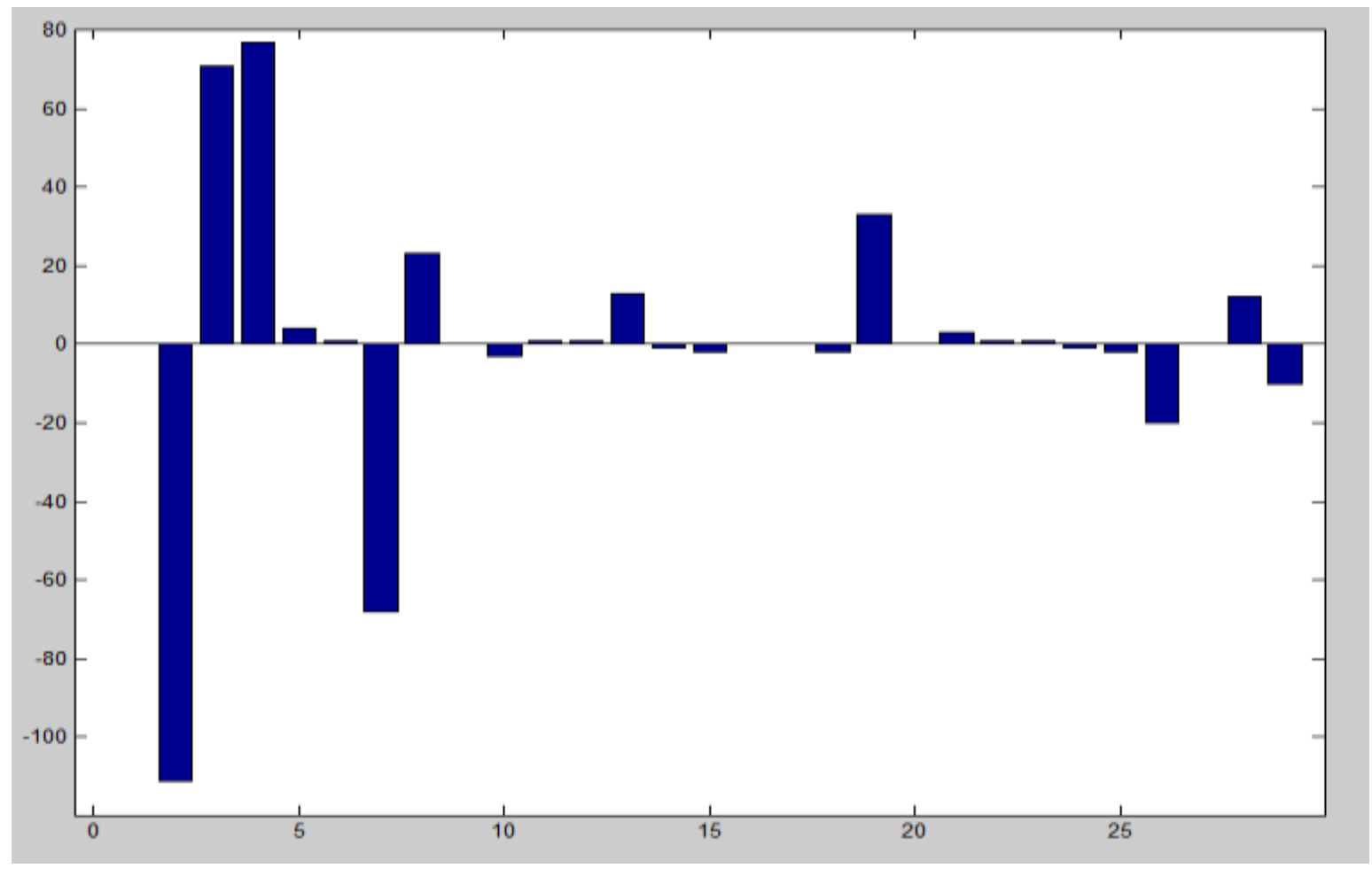

Figure 49 Day 1 Events vs. number of repetition

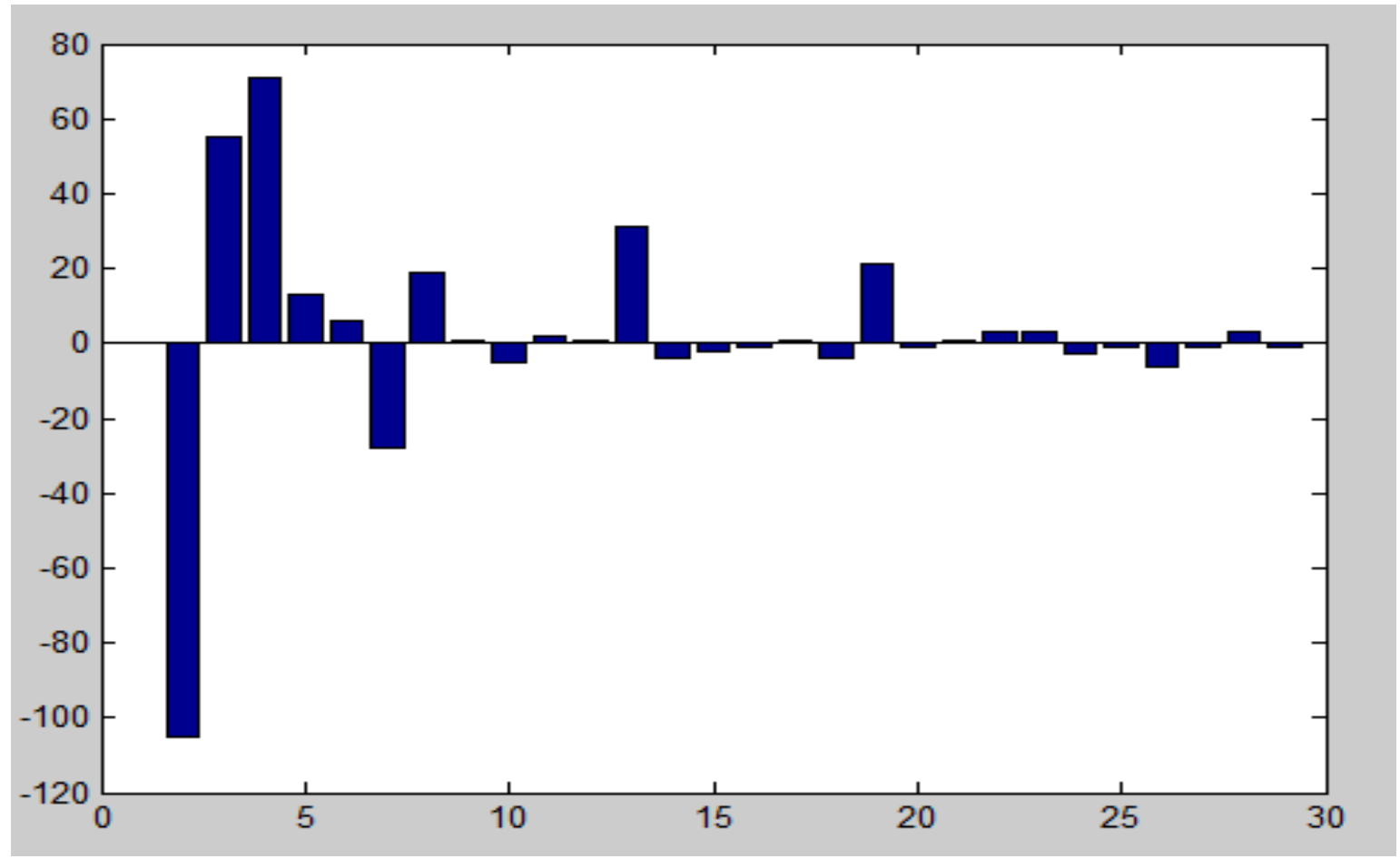

Figure 50 Day2 vs. number of repetition 


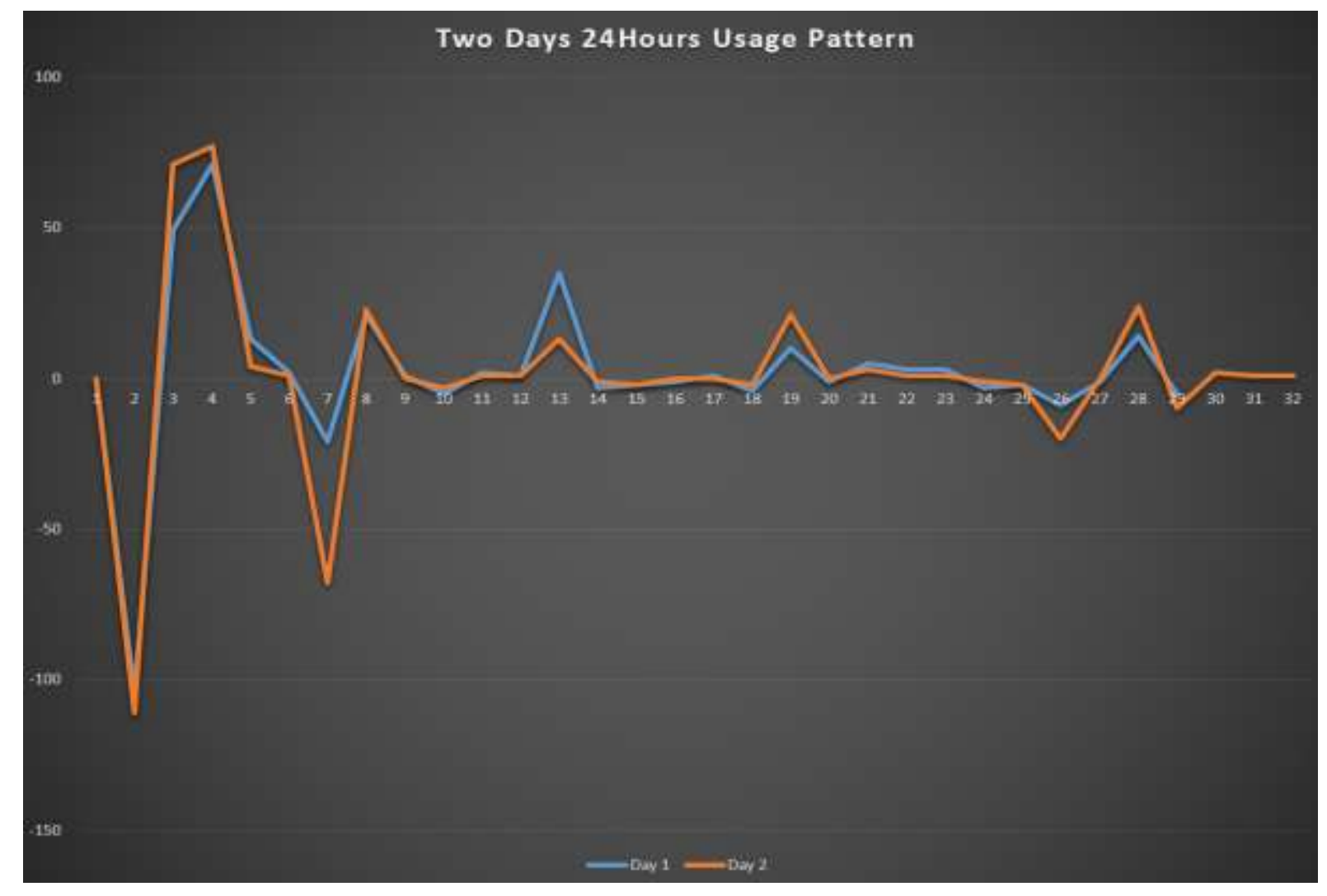

Figure 51 Appliances usage pattern for two days

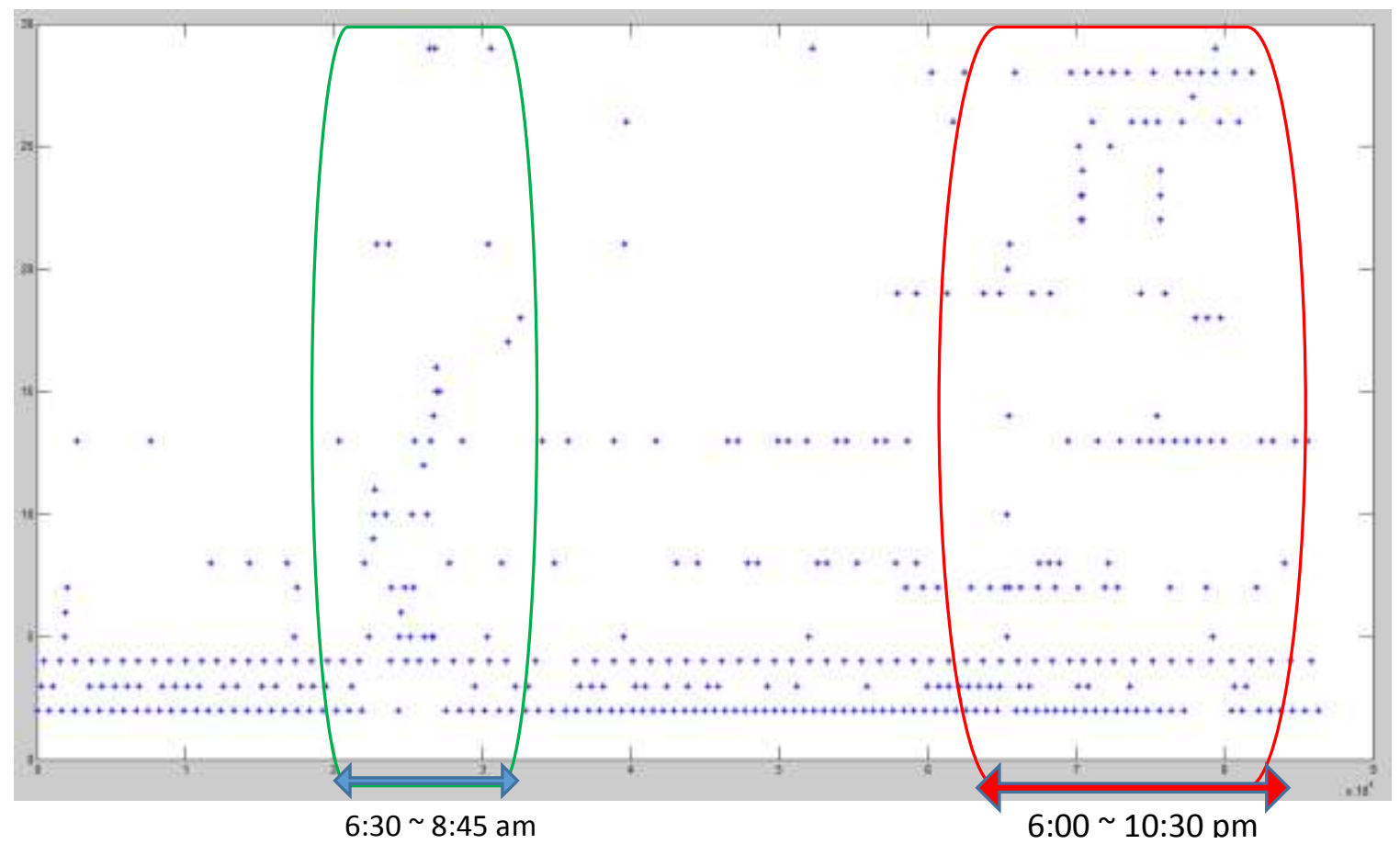

Figure 52 One day pattern for all events ( $V$ axis: event number, $H$ axis: time of day in second) 
Figure 52 illustrates one day ( 24 hours) data of a residential home. Inside the green line, which is about 6:00 in the morning to 8:45am, clearly it is shown there are more activities inside the home rather than during the day. The red line indicates the high level of activities occurring between $6: 00 \mathrm{pm}$ to $10: 30 \mathrm{pm}$. This result makes it very clear that for this particular resident, most of the activities are early in the morning and when he/she is coming back from work or school. There is not much activity between 8:45am and 6:00pm. It is obvious that still there are some other activities are seen during the day but most of them or probably all of them are due to the automatic control mechanism of different devices and appliances in home which do not require human interaction (e.g., air conditioner, water heater, automatic lighting system) and so on.

The main limitation of this project is detecting the OFF event of some appliances. Since low resolution signals are being used, and also because the signatures of OFF events for (different) appliances with a given power consumption are very similar, it is difficult or to distinguish between those appliances. This limitation can be overcome, however, because we are primarily concerned with the overall pattern of device usage and detecting the $\mathrm{ON}$ events is generally sufficient for this purpose.

Since the power signal used in this thesis has low resolution, we are not able to extract much information from it. Several approaches were tested that did not yield useful results. For instance, some devices have the same active and reactive power signature in the low resolution signals, along with almost the same overshoot, same average, and other similar features, thus preventing device discrimination. The problem of low resolution is even worse when we want to detect the turning OFF events, since most of appliances have a mechanical or electronic switch that will cut the power instantly at the point of turning off, 
precluding an obvious transient that can be easily characterized. Although it is still possible to detect some devices with a low resolution signal (as we have demonstrated), these low resolution signals do limit the range of features that we can practically extract for device detection.

Another limitation is detecting appliances that have power consumption near the threshold value. If large loads are being used, then the whole signal has fluctuations and the signalto-noise ratios of the signatures for the smaller loads are poor. Figure 53 shows two examples of this problem where the signal is not clear enough. The orange line is the power signature representation of the lamp without having any other loads online. In the right, it is the same scenario for the lamp when it goes OFF; the reactive power makes the signature not that accurate. As already mentioned, some modification is done in the code in order to improve this issue, but even so, detection of the loads near the threshold is not very accurate. Sometimes the system may not even detect those events since they may come below the threshold due to fluctuations and noise in the whole signal.

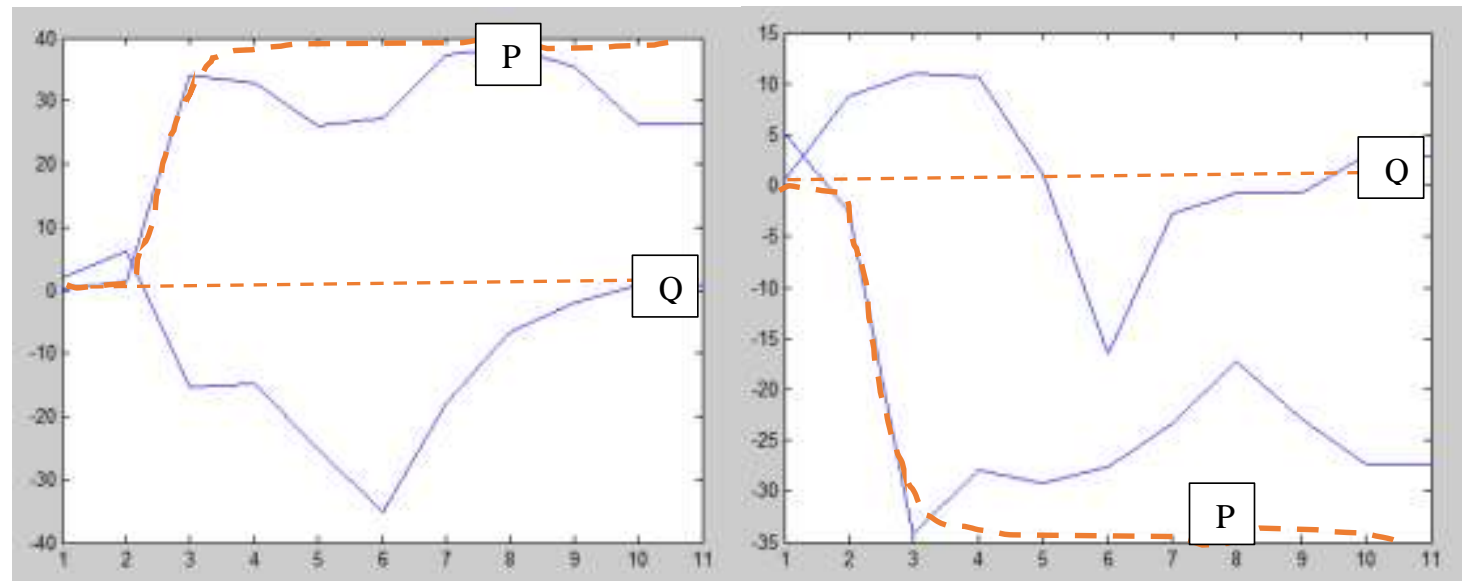

Figure 53 Left, Turn ON signature of a sample small load, Right: Turn OFF signatures of a sample small load 


\section{Future work}

\subsection{Current Systems}

The NILM Hardware used for our purpose in this thesis was able to collect data for analysis satisfactorily, but there is more room for improvement. Since this projects is intended to minimum the costs and also because of budget limitation there was no opportunity to examine different devices.

Although the analog-to-digital conversion by the Oscilloscope performed quit well, it was not very well and it could be changed with other tools to have more resolution data. A more advanced interface between the voltage and current transducers and the PC should also be investigated. High or low pass filters should also be considered at the interface between the transducers and the Oscilloscope.

In the other hand, Selecting and designing the right components and put them together can be a good idea, especially since in this thesis we were looking to find a way to transfer the data wirelessly. One idea can be designing and implementing a system to measure the signals of both voltage and currents and then transferring the signals to one of the analyzing software like Matlab or LabView preferably Matlab, since most of the calculation and analysis is doing in Matlab it would be a great idea to design and fabricate a cheap system 
that can connect to Matlab. If it could be possible to detect the devices status at the realtime it is much better to use LabView and if it is not then Matlab is the best choice.

One of the great idea since in this thesis tried not to use very complicated algorithm and low cost tools to detect the behavioral pattern then it could be possible to design a Micro Controller based with GSM enabled framework to do the all task from data acquisition part and also analysis part too. In that way it would be feasible to have this device with a reasonable cost to monitor elders and send alerts to a nurse or the organization that monitor their health.

\subsection{NILM Software}

The current state of the art in NILM software consists of the preprocessor that converts the voltage and current signals into a power envelope, the automatic data collection discussed in this thesis,

The other topic could be detecting the appliances status by finding the oscillating frequency like natural frequency of the power signal at the turning $\mathrm{ON}$ point, as it seems during this research, at the high sampling rate the power signal would be in a high resolution and then it is possible to look at the oscillations and vibration of signatures at the start point or when

they are online. In this thesis we are not considering the method that just mentioned but it could be a good research topic. 


\section{References}

6 Bibliography

[1] G. W. HART, "Nonintrusive Appliance Load Monitoring," IEEE, 1992.

[2] D. Rantz, "Computational Algorithms for Predictive Health Assessment," 2013. [Online].

Available: http://www.eldertech.missouri.edu/docs/Popescu\%20NSF\%20SHB\%20Summary.h tml.

[3] T. DeNucci, R. Cox, S. B. Leeb, J. Paris, T. McCoy, C. Laughman and W. Greene, "Diagnostic indicators for shipboard systems using non-intrusive load monitoring," in Electric Ship Technologies Symposium, 2005 IEEE, IEEE, pp. 413-420.

[4] H.-H. CHANG, "Load identification of non-intrusive load-monitoring system in smart home," in WSEAS Transactions on Systems 9.5, 2010, pp. 498-510.

[5] D. G. Hart, "Detecting drunk driving by NILM". Patent www.peertopatent.org/patent/20090063201/overview, November 2008.

[6] U. A. Orji, Z. Remscrim, C. Laughman, S. B. Leeb, W. Wichakool, C. Schantz, R. Cox, J. Paris, J. L. Kirtley and L. K. Norford, "Fault detection and diagnostics for 
non-intrusive monitoring using motor harmonics," in Applied Power Electronics Conference and Exposition (APEC), 2010 Twenty-Fifth Annual IEEE, IEEE, pp. $1547-1554$.

[7] Wikipedia, "Wikipedia.com," [Online].

[8] J. Paris, A Framework for Non-Intrusive Load Monitoring and Diagnostics, Massachusetts Institute of Technology S.M. EECS Thesis, 2006.

[9] E. R. Proper, "Automated Classification of Power Signals," MIT, 2008.

[10 S. M. I. I. K. N. a. R. W. C. I. Steven R. Shaw, "Nonintrusive Load Monitoring and

] Diagnostics in Power Systems," IEEE TRANSACTIONS ON INSTRUMENTATION AND MEASUREMENT, NO. 7, , vol. 57, p. 1445, JULY 2008.

[11 Ramsey, Jack S, Massachusetts Institute of Technology. Dept. of Electrical

] Engineering and Computer Science., "Shipboard applications of non-intrusive load monitoring," Massachusetts Institute of Technology, 2008.

[12 J.P.Mosman, "valuation of Non-Intrusive Load Monitoring for Shipboard Cycling

] System," Massachusetts Institute of Technology NE/S.M thesis, 2006.

[13 F. J. a. N. A. Taha Hassan, "An Empirical Investigation of V-I Trajectory Based Load

] Signatures for Non-Intrusive Load Monitoring," IEEE TRANSACTIONS ON SMART GRID, no. Aug 2013, p. 9, 2013. 
[14 P. Tech, "Pico Tech," [Online]. Available: http://www.picotech.com/picoscope3400-

] specifications.html. [Accessed 14 November 2013].

[15 "Dent Instruments," [Online]. Available: http://www.dentinstruments.com/.

]

[16 http:/hawkingtech.com/, "Hawking Tech," [Online]. Available:

] http://hawkingtech.com/. 


\section{TAI38 200 A AC Current Clamp}

OPERATING INSTRUCTIONS

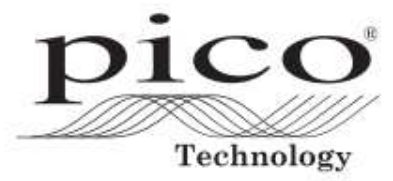

\section{Introduction}

The TA138 200 A AC Current Clamp has a voltage output with standard banana ( $4 \mathrm{~mm}$ ) plugs for connection to the PicoLog CM3 Current Data Logger or other compatible measuring instrument.

\section{Using the Current Clamp Safely}

Warning: To prevent electric shock or fire and personal injury, carefully read all safety information before attempting to operate the Current Clamp and follow these procedures:

- DO NOT use the clamp on circuits rated higher than $600 \mathrm{~V}$ in Category II or $300 \mathrm{~V}$ in Category III. Use caution when clamping around uninsulated conductors or bus bars.

- DO NOT use a clamp that is cracked, damaged, or has a defective cable. Such clamps should be made inoperative by taping the jaws closed.

- Do check the magnetic mating surfaces of the clamp jaws. They should be free of dust, dirt, rust and other foreign matter.

- Do keep your fingers behind the safety barrier as shown below.

\section{Safety Symbols}

This symbol, adjacent to another symbol or terminal, indicates that

1. This symbol, adjacent to a terminal, indicates that, under normal use, hazardous voltages may be present.

Double insulation

\section{Adaptor Description}

1. Current sense jaw

2. Clamp trigger

3. Safety barrier

4. Voltage output lead

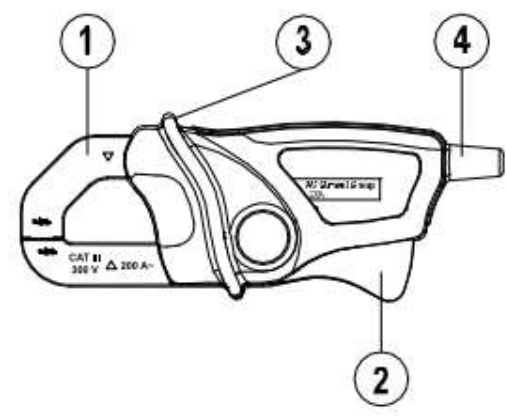

\section{Appendix A}




\section{Transducer Specifications}

\section{Specifications}

\section{- General Specifications}

Output impedance $<1 \mathrm{k} \Omega$

AC bandwidth 40 to $400 \mathrm{~Hz}$

Jaw opening $16 \mathrm{~mm}$

Operating conditions $32{ }^{\circ} \mathrm{F}$ to $86^{\circ} \mathrm{F}\left(0^{\circ} \mathrm{C}\right.$ to $\left.30^{\circ} \mathrm{C}\right) 90 \% \mathrm{RH}$; $86^{\circ} \mathrm{F}$ to $104^{\circ} \mathrm{F}\left(30^{\circ} \mathrm{C}\right.$ to $\left.40^{\circ} \mathrm{C}\right) 75 \% \mathrm{RH}$; $104{ }^{\circ} \mathrm{F}$ to $122^{\circ} \mathrm{F}\left(40^{\circ} \mathrm{C}\right.$ to $\left.50^{\circ} \mathrm{C}\right) 45 \% \mathrm{RH}$

Storage conditions $-22^{\circ} \mathrm{F}$ to $140^{\circ} \mathrm{F}\left(-30^{\circ} \mathrm{C}\right.$ to $\left.60^{\circ} \mathrm{C}\right)<90 \% \mathrm{RH}$

Altitude Operate at less than 3000 metres

Weight $129 \mathrm{~g}$

Dimensions $111 \times 50 \times 33 \mathrm{~mm}(\mathrm{H} \times \mathrm{W} \times \mathrm{D})$

Standards IEC1010-1 (1995); EN61010-1 (2001) Category II 600 V, Category III 300 V

\section{- Range Specifications}

\begin{tabular}{|l|l|l|l|l|}
\hline Function & Range & Output & Sensitivity & Accuracy (of reading) \\
\hline AC Current $(50 / 60 \mathrm{~Hz})$ & 0 to $200 \mathrm{~A}$ & $1 \mathrm{mV} / \mathrm{A}$ & $1 \mathrm{~A} / \mathrm{mV} \mathrm{AC}$ & $\pm 2.0 \% \pm 0.5 \mathrm{~A}$ \\
\hline
\end{tabular}

\section{Using the Current Clamp with the PicoLog Software}

1. Connect the output lead to the input sockets of the PicoLog CM3 Current Data Logger.

2. Connect the PicoLog CM3 to your computer using the USB cable supplied.

3. Position the Current Clamp perpendicular to and centered around the conductor.

4. Run the PicoLog software on the computer.

5. Select File > New Settings and configure PicoLog for a 'PLCM3' device.

6. Select File > New Data and click the 'Start recording' button when ready.

D0194-1 Copyright (c) 2011 Pico Technology Ltd.
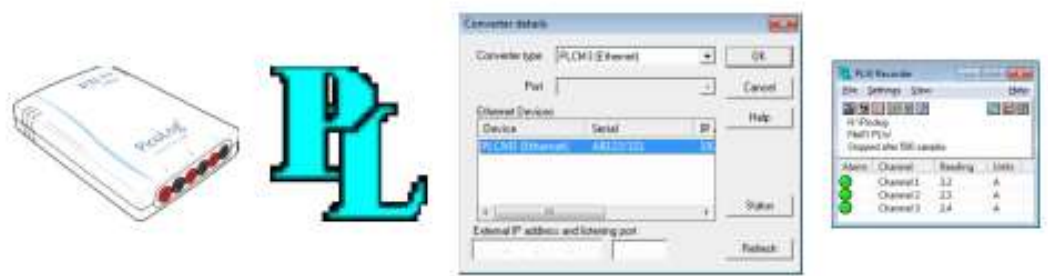


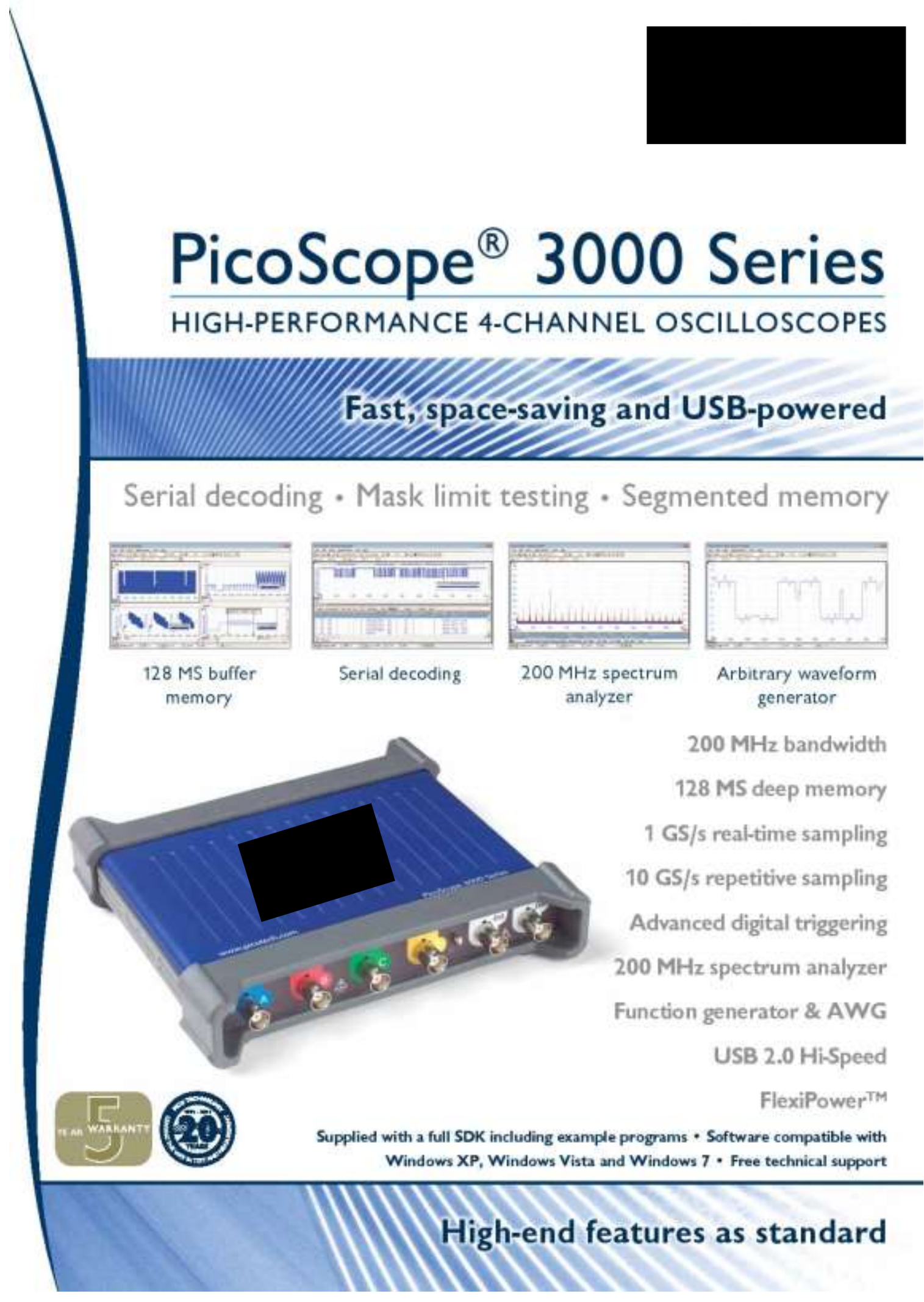




\section{PicoScope 3000 Series 4-Channel Oscilloscope}

\section{PicoScope: power, portability and versatility}

Pico Tectuchogy continues to push the lenits si USB-powered osclloscopes The new Feofoge 3000 Sernes offers the helest performance mallate

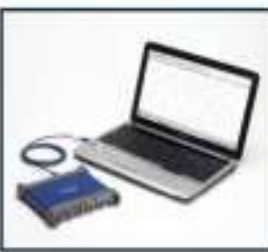
from any USB powered osilloscope on the market todty.

The Prooscope 3000 Series has the power and performance for many applicatons, such as design, reserch, test, edacation, service and repair

Pico USB-powered escilloscopes ar also smat, fisfit and portable. They easll slip into a laptop bag making the in idea for the enginetr de the move. The new Picoseope 3000 series 4-chamel osalloscopes feature Fexifowerty siving you the option of powering the scope from two USB ports, so you can inse the power supply behind when using the devict in the fiełd

\section{High bandwidth, high sampling rate}

Unlike most USB-powered ascilloscopes, with red-time sampling ratr. of only 100 or $200 \mathrm{MS} / \mathrm{s}$, the Pleoseope 3000 Series delwers a marketleading $1 \mathrm{CS} / \mathrm{s}$. ETS mode boosts the maximum effective sampling rate firther to $10 \mathrm{GS} / \mathrm{s}$, enabliag even fiver time reschution when used with repetitive signals.

\section{Deep memory}

The Acoscope 3000 senes offers memory deptis is to 128 mallion samples, more than any otker ascilloscopes in this price range.

Other osolloscopes hwe high madumum simpling rates, but withicest deep metrory they cannot sustain these rates on lowp timebasts. The Prossmpe 34068 can sample at $1 \mathrm{CS} / \mathrm{s}$ at timehases all the way down to $10 \mathrm{~ms} / \mathrm{dr}$.

Manegine all thes data calls far some powerfid tools, so PooScope has 4 maximum zoom factor of 100 million combined with a dhexes of twe zocm methods. There's a conventional set of zoom controls, plus as overview sindow that shows you the whole wweform whle you zoon and reposition the display by sinply drageing, with the moust.

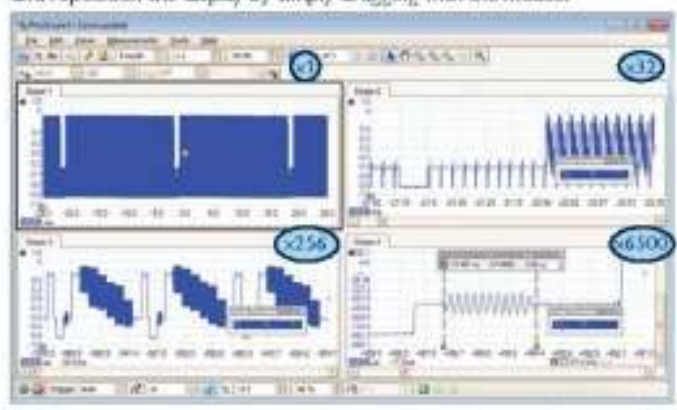

The deep meinory can be segmented to store multiple waveform, and has navigation tools allowing you to review up to 10,000 previous entries. No longer will you see a plitch on the screen only for it to vanist before you stop the soope. A mask can be applied to filter cat wsveformis of interest.

\section{Advanced triggers}

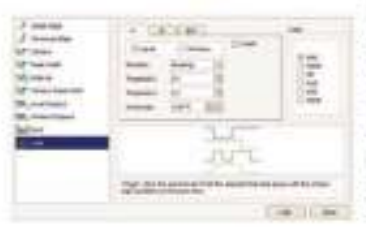

As well as the standard range of triszers found on all osciloscoges the Pcoscope 3000 Series offer: a class leading set of adranced triggers including pulse width windowed, dropout and logic triggers to help you capture the data you need.

\section{Digital triggering}

Most digitz osdiloscopessold today still use an malog trieger architecturt based on comparacors. The can cisive time and amplitade erron that cannot always be calitrated out. The use of coniparators aften limits the. triger sensitivity at begh bandwidths and can also create a long theger "re-armi" delay.

Snce 1991 we have been pioneering the ase of fully digital triggerint using the actual digitized dath. This reducts trigger erroes and allows cour oxilloscoges to trieger on the sinvilest seanals, even at the full buatwidth Trieger levels and tysteresis can be set with tigh precision and resolution.

Degital viesering also reduces re-arm delay and this, combined with the sepmented memory allows the tripzering and capture of events this happen in rupid sequence, At the fastest timebase you can use rapd triegering to called 10,000 waveformn in under 20 millineconds, The mast limit testing function can then scx theough these wweforms to helphegh any failed waveforms for viewing in the sriveform buffer.

\section{Custom probe settings}

The castom probes feature vilows you to cerrect for guin, attemation. offsets and nonlinearities in special probes, $\alpha$ to convert to different unit: of meassement (wuch as currefit, power or tempernture) You cus swe deffritions to disk for later use. Befinitions for Pico oscllloscopt probes and aurtent demps are tailt in.

\section{Arbitrary waveform and function generator}

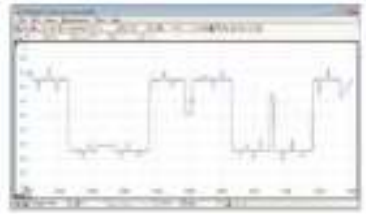

All units have a built-in function generator (sine, square, tnasele. DC level) with frequency sweeping capability, Combined with the spectrum peak hold option, this makes a powerfin tool for testing amplifier and fitter responses.

The B mosels in the Picoscope 3000 Series also include a full arbatrary waveform-generatoe. Wweformas can be crested or modified using the beilt in AWG edtor, imported from osclioncope traces, or losded from a spreadshet.

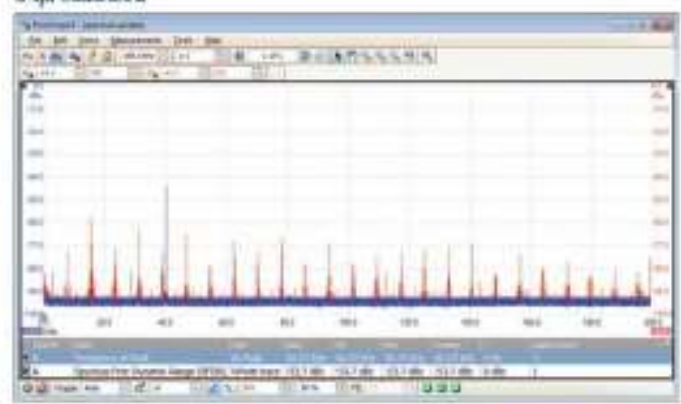

\section{Spectrum analyzer}

With the didk of a button you can display a spectrum plot of the selected donnek. The spectrum ambyzer allows sigraks up to $200 \mathrm{MHz}$ to be viewed in the firequency domain, A full range of settings gives you control over the mimiber of spectrin bandk, window type and display mode: instuntineous, merape, or peik-fwold.

You can dsplay nuiltiple upectrum views with different danenel selection and zoom factors, and place these alongside time-domain views of the same data. A comprehensive set of autamatic frequency-domsin measurements, includine THO, THD+N, SNR, SNAD and IMD, can be added to the dasplay. 


\section{Advanced display modes}

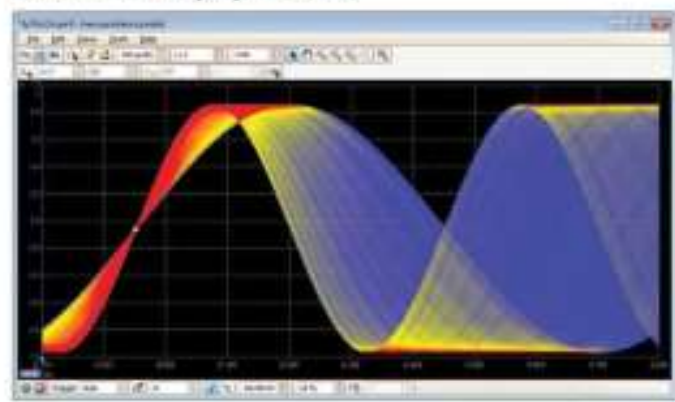

See old and new data saperimposed, with new data in a brighter color of shade. This makes it easy to see glitetes and dropouts and to estimate their relative frequency. Choose between analos persistence and disital color, or create a custom dixplay mode.

The design of the Picoscape software ensares that meximam dicplay area is walubile for wiveform viewne, Even with a haptop you hwe a mach bejger viewing area and tigher resolution than a typoical bencheop acope.

\section{Serial decoding}

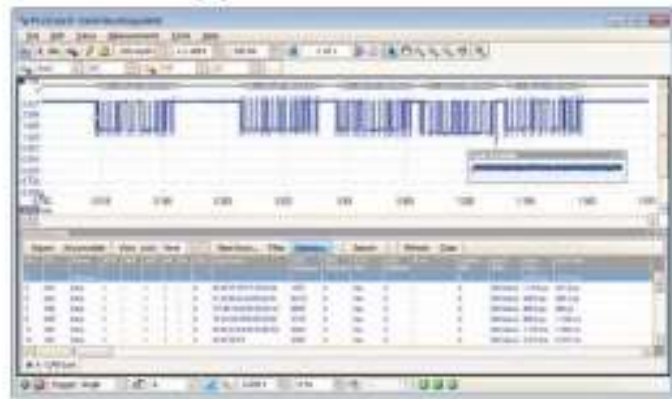

The deep memary Hooscope 3000 Series is ideal for serial decoding as it can capture thousands of frames of uninterrupted data.

Protocols currenly supported are FC, SH4, RS232/UARI, CAN, LIN and Flexiay. Expect this list to grow with free sxleware updates.

Picoscope displays the decoded data in the format of your choices "in view", "In window", or both at once.

"In vew" format shoms the decoded data beneath the waveform on common nime axks, with error frames marked in red. You can zoom in on these frames to lock for noise or distorbon on the wweform. "In window" format shows a list of the decoded frames, insluding the dat: and all flags and identifiers. You can set up filtering condtions to display caly the frames you are interested in, search for frames with ipecified peopertis, or defint a start pattern that the proeram will wait for before listing tie das. Yau can also create a spreadshet to fully decode the hes data into plain vext.

\section{High-speed data acquisition/digitizing}

The drivers and onftware develogment kit supplied allow you to write your own software or interface to popular therd party software packages whas LabVEW,

If ste 128 M5 record length isn't encught, the driver supports streaming mode, which captures gap-free cominuous data trough the USB gori directly to the PC's HAM or hard dak at a rate of over $10 \mathrm{MS} / \mathrm{s}$. Maximum speed depends on the PC's capabilaties.

\section{Mask limit testing}

This featare is specially designed for production and debaggent environmeats. Captare a signal from a known workine system, and Picofcope wifl driw a mask around it with your soecofied toterance. Connect the system under test, and Pscofcope will bighight any parts of the wavelorm that fall outside the mask area. The liglighted decail persist on the dsplay, allowine the scope to catch intermituent glitdres whle you work on sonxtiang else. The measurements window cosant: the mumber of faibres and can dsploy other measurenents and statistics at the same time,

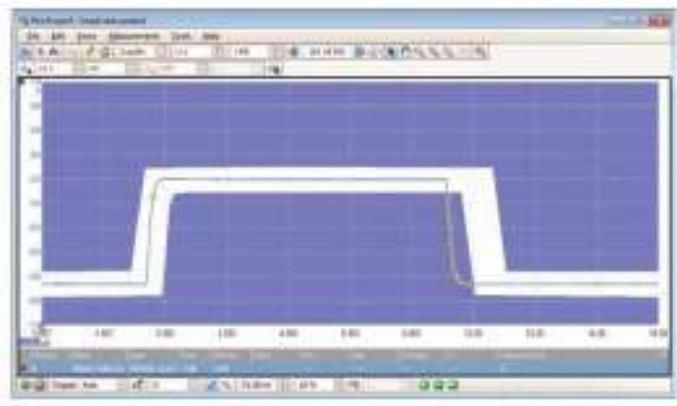

The numerical and eruptical musk editors can be used separately of in combinution, allowing you to enter amairier inask spedifations and to modify existing maks. You can import nd export moks as files.

\section{High-end features as standard}

Buying a scope fecm some comparies is a bit libe buying a car. By the time you have added all the optional extras yoe need, the price han gone ap consider bly. With the Preoscoge 3000 Series helh-end features such an mask lemet testing, serial decoding adkanced triggering, measurements. insh, $X Y$, deital fitering and segenented memory are all induded in the price.

To protect your investment, both the PC sofitware and firmware hiside the unit an be updated. We fave a long history of providag new features for free vis software downloads. Other companies make vazue pronkses sbout future entancements but we deliver on our promises year after year. Users of aur products reward us by beconing lifelone customers, frequently recommending es to their colleagues.

\section{Dependable signal integrity}

Most oscilloscopes are buile down to a price; ours are beilt up to specification.

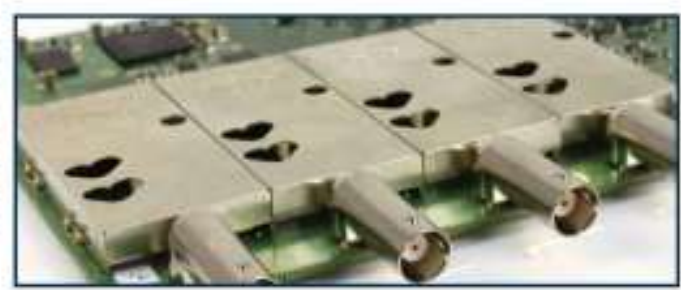

Carcful framt-end desion and shiebding reduces noise, crosstslk and turmonic distortion. Years of ondiloscoge experienot leach to impronted polse response and bandwidth flatness.

We are proud of the dynamic performance of cor prodacts and publst these spedifications in detall The result is simples when you probe a drcait, you cas irust in the waveform you see con die screen. 


\section{PicoScope 3000 Series 4-Channel Oscilloscopes - The PicoScppe pilp}

Oscillos cope controlst Commonly-used controls such as voltege range velection, timebase, memory depeh and chanel selection are placed on the toobar for quick access. le sing the min display area dear for waveforms. More advanced cronitrols and finetions se located in the Toob memi.

Tools $>$ Math channels: Combere inpot damek and reference waveforms using simple scitimetic. or create oustom equations with ir konometric nd other functions.

Tools S erial decoding: Devode nultople serial ifata sienals and dikplay the data alongside the phonical sigesal or wa detailed table.

Tools Reference chaninels Store waveforms in memary or on disk and display them alongside live inputs, ideal for diagnostics and prodiction testing.

Auto setup buttonr Confifures the triebrse and voltapt rumges. for statile display disiesals.
Picoscopet tix display ea be as simple er as coniplex as you need. Besin with a single view of oret chanet, and then exprad the dsplar to include any namber of live chunnels, muth divinets and reference waveforms

Waveform replay tool: Picoscope autonsuically records ip to 10,000 of the most recent wareforms. You cun quickby san through to look for interminent events.

Viewe Frafoope is carefully designed to mike the best use of the display area. You can add new scope and spectrum views with watomatic or oustan layouts.

Rulersi Each axis has two rulers that can be drugged across the screen to make quick messurements of amplitude, time and frequency.

Zoom and pan toolss Preofecope allows a zcom facter of up 100 million, which is nectessary when working with the deep memary of the 3000 Series scopes. Eather use the zoom int, zoont-out and pan tooks, or click and drag in the zoom overview window for fast navipation.
Movable axess The vertical axes can be draged up and down. This featare is par bicularly uneful when one waveform is obscanne another. There's also an Auto Arrange Axes command.

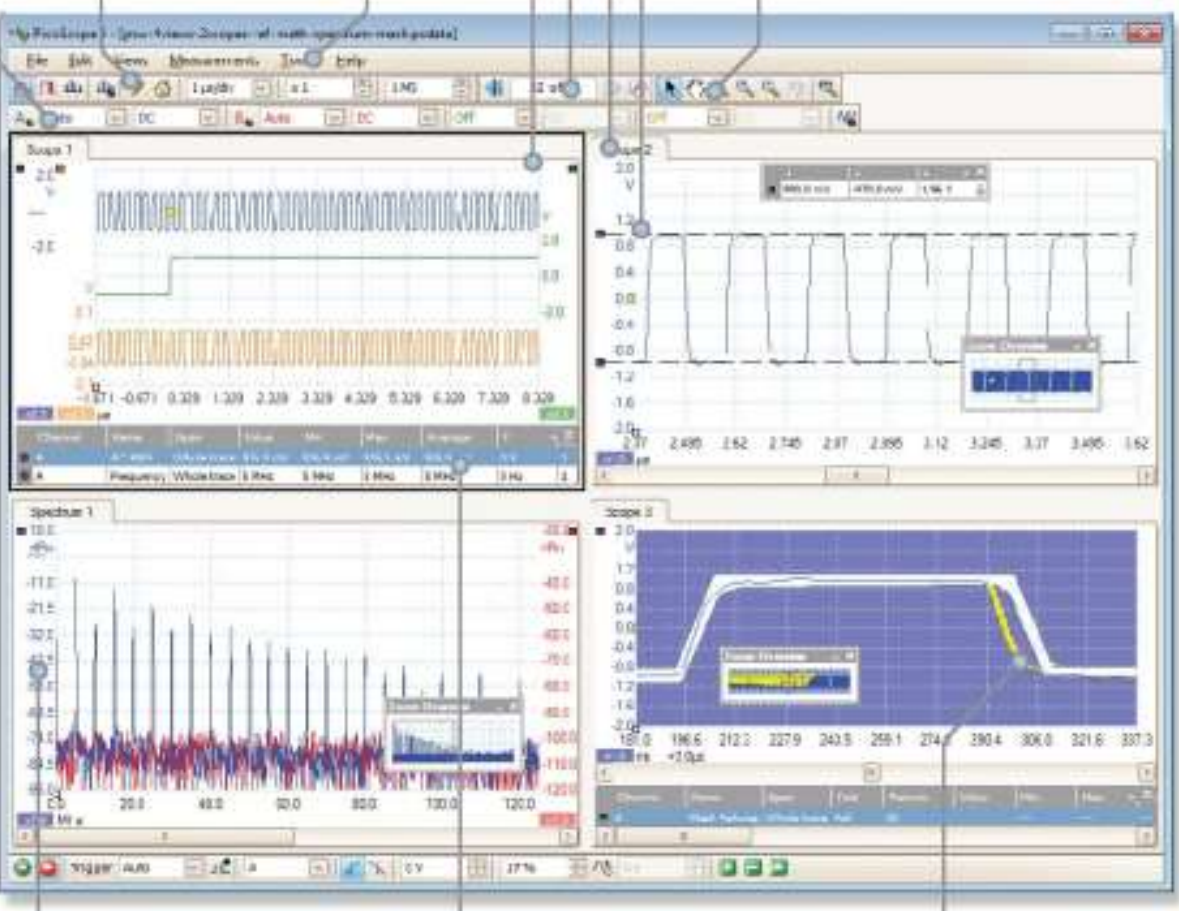

Automatic measurementst Desplay calculated measurements for troublestiooting and andyaih. You can add as mamy memurements as you need on each view. Each messar ement includes stadistical paraneiers showing its variabitit:
Mask limit testing: Automatically generate a test mask from a waveform of d'raw ane by hand. Pcoscope highights any parts of the waveform that fall onitside the mask and shows error itatistics. 
PicoScope 3000 Series 4-Channel Oscilloscopes - Specifications

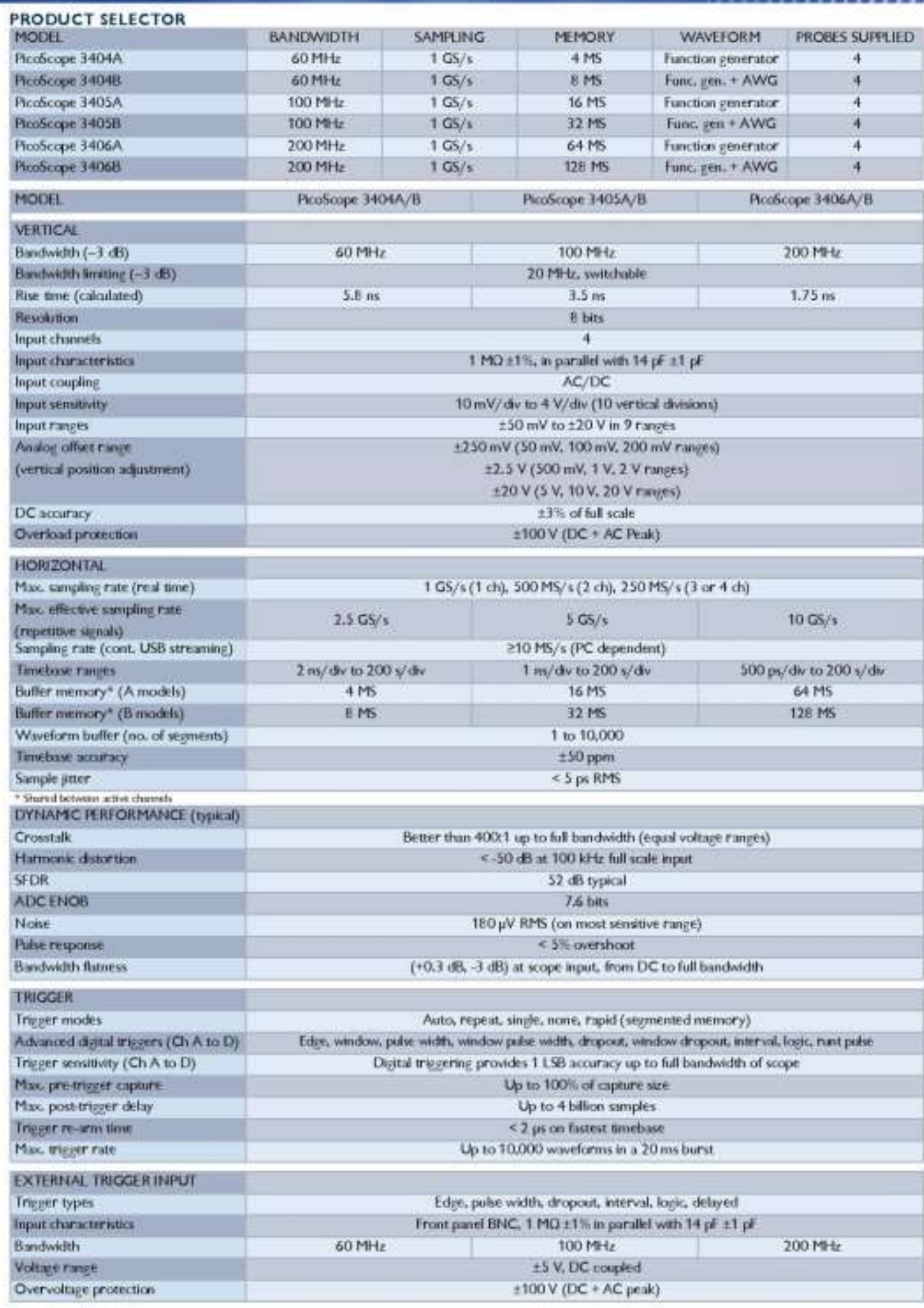


PicoScope 3000 Series 4-Channel Oscilloscopes - Specifications (continued)

\begin{tabular}{|c|c|c|c|}
\hline MOOH & Fico5cope 3404A/B & Кrooscope 340SA/B & Acosrope 3406/B B \\
\hline \multicolumn{4}{|l|}{ FUNCTION GENERAIOR (al modeds) } \\
\hline Qutput winedons & \multicolumn{3}{|c|}{ 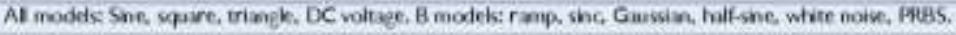 } \\
\hline Oulput frequency range & \multicolumn{3}{|c|}{ DC to $1 \mathrm{MHz}^{2}$} \\
\hline Swerp modes: & \multicolumn{3}{|c|}{ Up, down, dus/ with sefectuble start/stop frequencies and increments } \\
\hline Asadvilith & \multicolumn{3}{|c|}{$>1 \mathrm{MHe}$} \\
\hline Qutput frequeng accuracy & \multicolumn{3}{|c|}{ \pm 50 ppm } \\
\hline Output frequency resolution & \multicolumn{3}{|c|}{$<0.01 \mathrm{H}$} \\
\hline Output voltage range & \multicolumn{3}{|c|}{ $\pm 2 \mathrm{~V}$ wth $\pm 12 \mathrm{DC}$ accuracy } \\
\hline Output vedtage adustriens & \multicolumn{3}{|c|}{ Senal ancilude and offset adustatie in approx, $1 \mathrm{mV}$ steps wetin overal $\pm 2 \mathrm{~V}$ range } \\
\hline Ampltude flatorss & \multicolumn{3}{|c|}{$<0.5$ di to 1 M/tz, typical } \\
\hline SFDR & \multicolumn{3}{|c|}{$>60 \mathrm{cB}, 10 \mathrm{kHz}$ full scale sine wrive } \\
\hline Connector type & \multicolumn{3}{|c|}{ Froest panel BNC with $600 \mathrm{Q}$ outpat mpednce } \\
\hline O.ervitage protentina & \multicolumn{3}{|c|}{$+10 \mathrm{~V}$} \\
\hline \multicolumn{4}{|l|}{ AWG (B models only) } \\
\hline Update rate & \multicolumn{3}{|c|}{$20 \mathrm{~m} / \mathrm{s}$} \\
\hline Buffer size & $8 \mathrm{ks}$ & B KS & $16 \mathrm{kS}$ \\
\hline Resolution & \multicolumn{3}{|c|}{12 bits (output step stat spproik, $1 \mathrm{mV}$ ) } \\
\hline Axidwitth & \multicolumn{3}{|c|}{$>1 \mathrm{Mte}$} \\
\hline Rise tme $(10-90 \%)$ & \multicolumn{3}{|c|}{$<100 \mathrm{~ns}$} \\
\hline FROE COMFENSATION OUTFUT & \multicolumn{3}{|c|}{$1 \mathrm{kHz}$ square wave, $1.6 \mathrm{~V}$ pkpk (typ), $6(0) 0$} \\
\hline
\end{tabular}

\begin{tabular}{|c|c|c|c|}
\hline \multicolumn{4}{|l|}{ SFECTRUM ANALYZER } \\
\hline Freguencr range & DC to $69 \mathrm{MHz}$ & DC to $100 \mathrm{MH}$ & DC to $200 \mathrm{MH}$ \\
\hline Display mods & \multicolumn{3}{|c|}{ Magitude, avtrage, penk hold } \\
\hline Whidowing finctions & \multicolumn{3}{|c|}{ Rectangular, Gakssin, trixapuar, Blackman, Blackmantharns, Hamming, Hann, fhe top } \\
\hline Number offFI poants & \multicolumn{3}{|c|}{ Selectable from 128 to 1 nillion in powers of 2} \\
\hline
\end{tabular}

\begin{tabular}{|c|c|}
\hline \multicolumn{2}{|l|}{ MNHCMNNAS } \\
\hline Functbons & 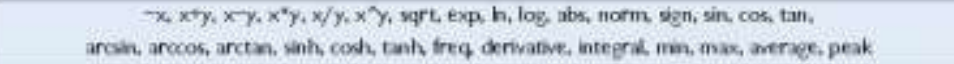 \\
\hline Operands & A, B, C, D (input daninek), T (dime) neference waveforms, constants, pi \\
\hline \multicolumn{2}{|c|}{ AUTOMATIC MEASUIEMEETIS } \\
\hline Oxiloscope & $\begin{array}{l}\text { AC RMS, true RMS, DC average, cyck ume, frequency, duty cyde, filing rate, fill time, } \\
\text { eising rate, fise time, high pulse wioth kow pube width, maximum, minimam, peak to pesk. }\end{array}$ \\
\hline Sptctrum & $\begin{array}{l}\text { Frequency at peak, amplitude at peak, avernge ampleude at peak, } \\
\text { total pomer. THD \%, THD dB, THD-N, SFDR. SINAD, SNR, IMD }\end{array}$ \\
\hline Statiktics & Mrimum, moxkiam, werage and stunded devation \\
\hline SERML DECODING & CAN, UN, Flextly, IHC. SH, and RS232/UART protocols \\
\hline MASK LIMII IESTING & Statisticer pasy/foil, failure count, total count \\
\hline \multicolumn{2}{|l|}{ DISHAY } \\
\hline Interpolvion & Lines or $\sin (x) / x$ \\
\hline Persistence modes: & 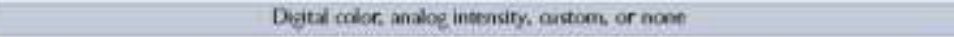 \\
\hline \multicolumn{2}{|l|}{ CENERAL } \\
\hline PC connection & USB 20 hrspeed \\
\hline Pover requirements & Pontered from 2 USB ports or from AC adaptor sapplied (1000 mA st $5 \mathrm{~V}$ ) \\
\hline Detentensions & $190 \times 170 \times 40 \mathrm{~mm}$ (including saniectons) \\
\hline Weith & $<0.5 \mathrm{kE}$ \\
\hline Temperisure range & Operating: $0^{\circ} \mathrm{C}$ to $40^{7} \mathrm{C}\left(20^{\circ} \mathrm{C}\right.$ to $30^{\circ} \mathrm{C}$ for stated acrunary $)$ \\
\hline Safery approwak & Desiged to EN $610104: 2010$ \\
\hline EMC sprionals & Tested to EN61326-1:2006 and FCC Pirt 15 Subprit B \\
\hline Enironimentel approvals & RoHIS and WEtE compliant \\
\hline Software/PC requirements & Acoscope 6, SCK and exangle proerams. Marosctt Whidows XP. Whadows Visa or Windows 7. \\
\hline Lanquages (ful support) & English. French, German, Italim, Sprish \\
\hline Languages (Ut only): & 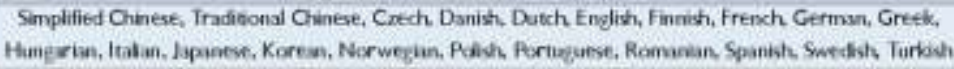 \\
\hline
\end{tabular}




\section{Connections}

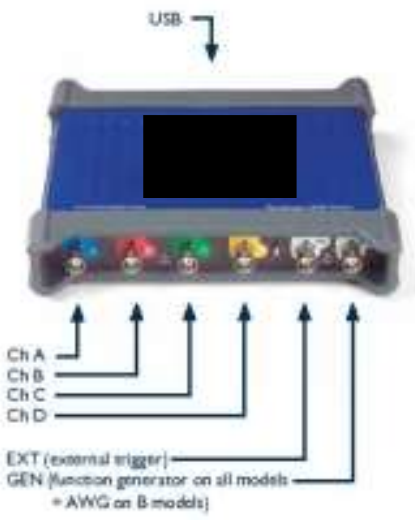

Software Development Kit

The Pcoscage 3000 Series SDK is avallable for free downiloud is contuins domers and programming examsiples in the folloneng tanguapes and development environments: - $\mathrm{C}$

- Ca

- Excel

- Labview

\section{Kit contents}

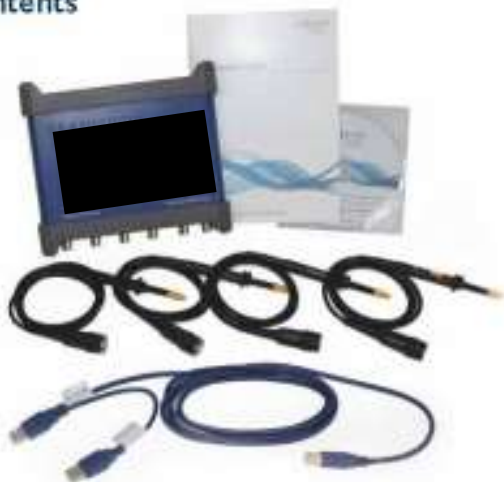

Your Psooscope 3000 Series osclloscope kit contains the following itens:

- Mocobcope 3000 Senes os dilloscope

- $410: 1$ probes

- Single-headed US8 cable

- Couble-teaded USB cable

- AC power adoper

- Installution Guide

- Softwire and Refereice CD
High-quality probes

The probes surppled with these osilloscopes are chosen to abtain the specitied sntem bandwidh

- MO07 (wapplied whi Acoscope 3404A/E)

- IA132 (supplied wath Pico5cope 3405A/B)

- TA131 (supplied with Accoscape 3406A/B)

\section{Ordering information}

\begin{tabular}{|l|l|c|c|c|}
\hline ORDER CODE & DESCRIPTION & $\ell$ & USS* & $\epsilon^{*}$ \\
\hline PP846 & PicoScope 3404A (60 MHz, func. gen., probes) & 599 & 988 & 725 \\
\hline PP847 & PicoScope 3404B (60 MHz, func. gen.+AWG, probes) & 749 & 1236 & 906 \\
\hline PP848 & PicoScope 3405A (100 MHz, func. gen., probes) & 899 & 1483 & 1088 \\
\hline PP849 & PicoScope 3405B (100 MHz, func. gen.+AWG. probes) & 1049 & 1731 & 1269 \\
\hline PP850 & PicoScope 3406A (200 MHz, func. gen., probes) & 1199 & 1978 & 1451 \\
\hline PP851 & PicoScope 3406B (200 MHz, func. gen.+AWG, probes) & 1349 & 2226 & 1632 \\
\hline
\end{tabular}

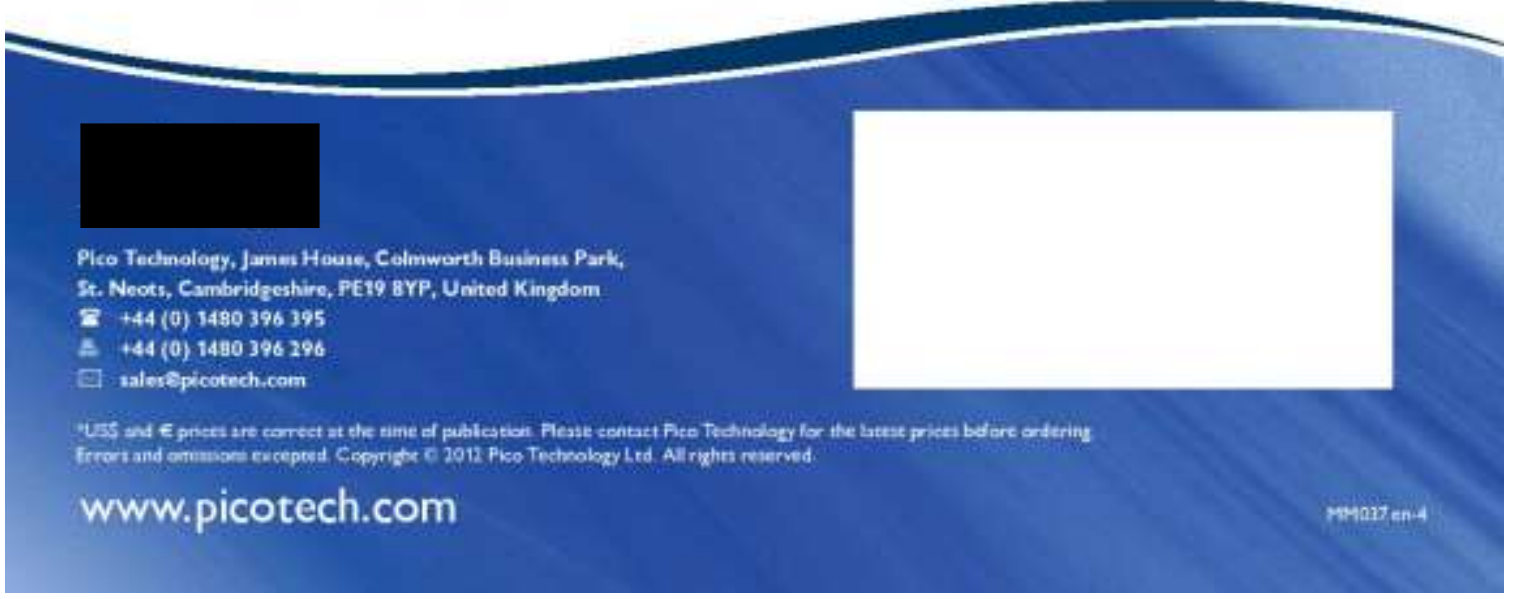




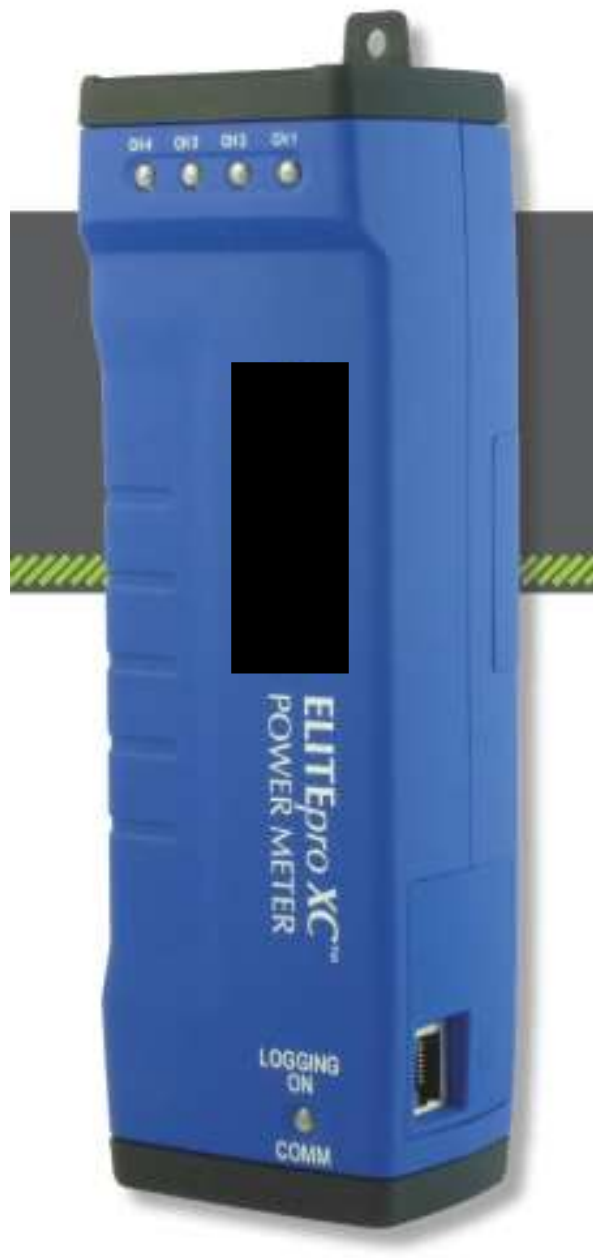

\section{ELITEPRO X $\mathbf{C}^{\mathrm{m}}$ PORTABLE RECORDING POWER METER}

\section{THE NEXT GENERATION ENERGY LOGGER} FROM DENT INSTRUMENTS

\section{FEATURES}

- For single or 3-phase systems using 80-600V phase-to-phase $(A C \mathrm{C}$ or DC7 services when ine powered or $06000 \mathrm{~V}$ (AC or DC) when externaly powered. Measures up to four chansels of energy metrics with currents ranging from 0-6,000 amps.

- Anvog irouts record anviog data with confecurable input ranges for votage $10-10$ $V D C l$ or curtent loop $(0-20$ or $420 \mathrm{~mA})$ transiducers. Analog inouts are used for process $\alpha$ erwrumental carrebition studes with power.

- Linepowered - no need for batteries or external power source

- User selectable recorting intervals as short as every 1 second

- $16 \mathrm{MB}$ nonvolatile memary standard for months of recording tme.

- Better than 18 accuracy $1<0.2 \%$ typical

- Fast US8 connection and standard Ethemet part.

- EUTEpro XC may be configured with optional Bluetoont" wireless technology

- Ootionat WFFi interface allows for remote data collection over wreless networks. Check reaitime vakues using a Wiflenabled laptop, tablet, of smartphone via a web browser.

- Sample yet powerful Wridows based ELOG' soltware package for setup, data retrieval, and analysis. Easy data exporting to amost any analysis program.

- Easy to setup and installs in mirutes. Patented PhaseChek" LED indicators ensure correct CT orientation at instalation.

- Rugged and combact-easily fits inside breaker parels and switch gear.

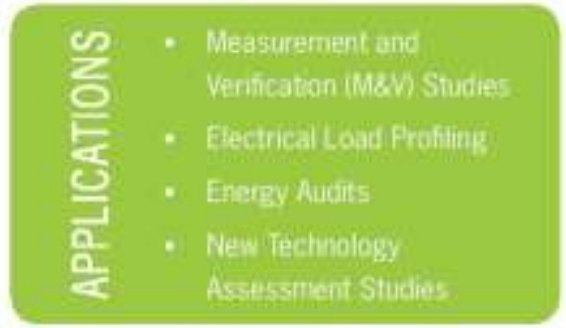




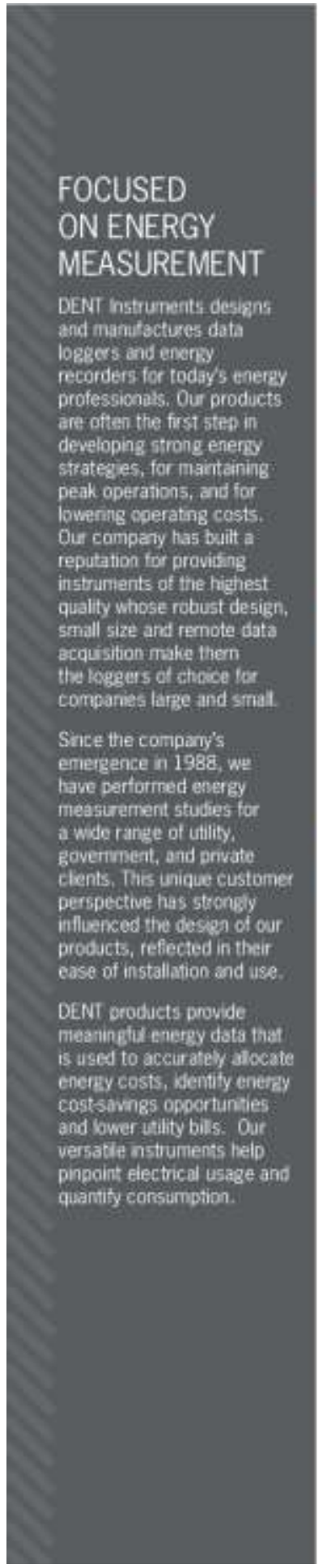

\section{ELITEPRO XC"'DETAILS}

\section{A DEPENDABLE AND VERSATILE TOOL TO MEASURE YOUR ENERGY USAGE}

The EUTEpro XC is a complete solution for pinpointing electric usage and quantifying energy usage. It is capable of measuring, storing, and analyzing electrical consumption data which is derived from the voltage and current inguts. The EL.TE Em XC uses direct connections to each phase of the voltage and various interchangeable CT cotions such as splitcore current transformers or flexble RoCoils ${ }^{1 \mathrm{M}}$ (for large loads or large cables and bussbars) to monitor current on each phase.

These meters can capture KWHYW energy and demand data as well as virtually all relevant energy parameters for diagnostics and moritoring on ttreephase or single-phase circuit instalations. Electrical lbad dagnostic parameters, such as displacement power factor, are captured in adidtion to energy and denand values.

The EUTEpro XCs s fexibility, size, and ease-of-use make it the ideal toal for gathering detailed consumption information in commercial, industrial, government and retail environments.

\section{FOUR ANALOG INPUT CHANNELS: CORRELATE YOUR CONSUMPTION WITH ENVIRONMENTAL CONDITIONS}

New to the EUTEpro XC are 4 analog input channels which can be configured for voltage or cursent input used in any combination among channels, Anatog inputs are especially helpotul when used in conjunction with power measurements to correlate the consumption of electricity with environmental, HVAC plant performance, or other process conditions. Typical uses migit include logging ambient temperature, building temperatures, solar insolation, tank pressure-5, duct flow5, etc. Obtain this valuable data using just one meter: the ELITEpro XC.

\section{ELITEPRO XC ANATOMY}
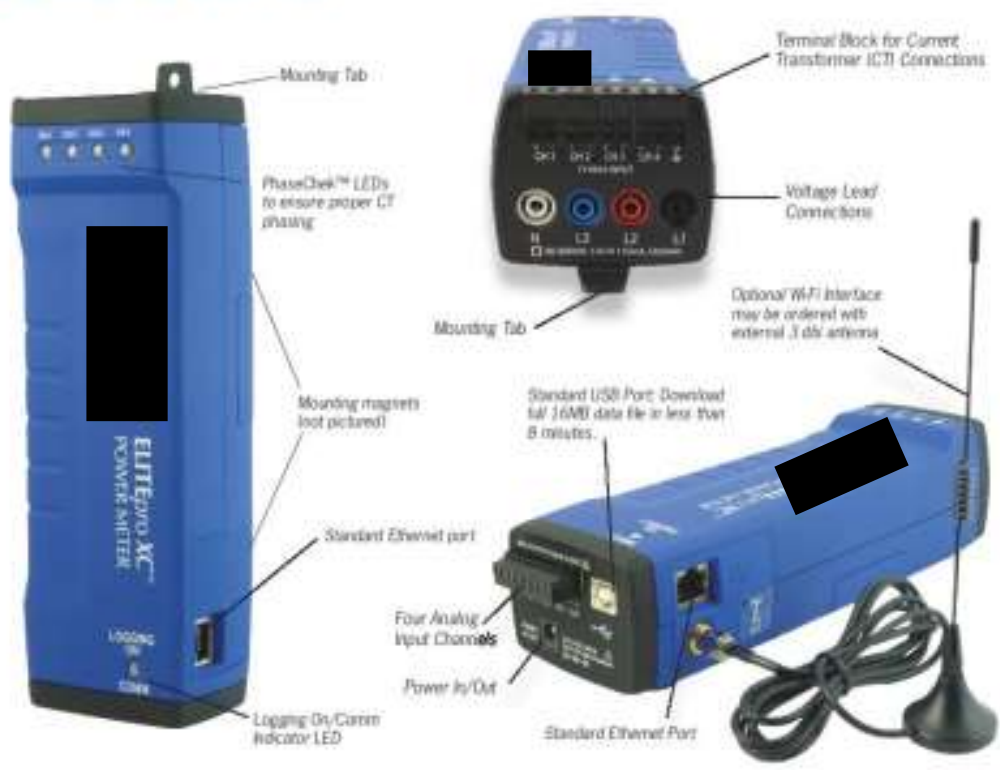


\section{ELITEPRO XC" FEATURES}

\section{LINE POWERED WITH REDUCED IMPACT ON THE ENVIRONMENT}

The EUTEpro XC is powered directly from the phases of the service being measured. It incorporates a broadband power supply which operates on virtually any $80-600 \mathrm{~V}$ phase-tophase service. You will not have to worry about constantly changing or recharging batteries, nor finding an external power source at the job site. And you will awoid the negative environmental impact of disposing of batteries containing rare earth materials that are difficult to recycle or reclaim.

\section{MEMORY FOR EXTENDED RECORDING}

Measurements are stored ir on-board memory at recording intervals selected by the user, which can be as stort as one second or as long as once every 24 hours. The ELIIE pro $X C$ has $16 M B$ of internal nonvolatile memory, which allows for long-term logging sessions. For example, the EUTEpro XC can record a WYE sefup with all avalable system measurements using a 2 minute integraton interval for over 160 days!

\section{FAST SAMPLING RATE DISPLAYS POWER QUALITY DATA}

Regardless of the user-selectable recording interval, the ELTTEpro XC has a waveform sampling frequency of $12 \mathrm{kHz}$. This is the number of data points the logger samples on each AC waveform (200 points per cycle at $60 \mathrm{~Hz} ; 240$ points per cycle at $50 \mathrm{~Hz}$. This fast samping rate alows for reat time display of voltage, current wavetorms, and harmorics. The charnel samipling tate is $8 \mathrm{~Hz}$ or every $125 \mathrm{mSec}$
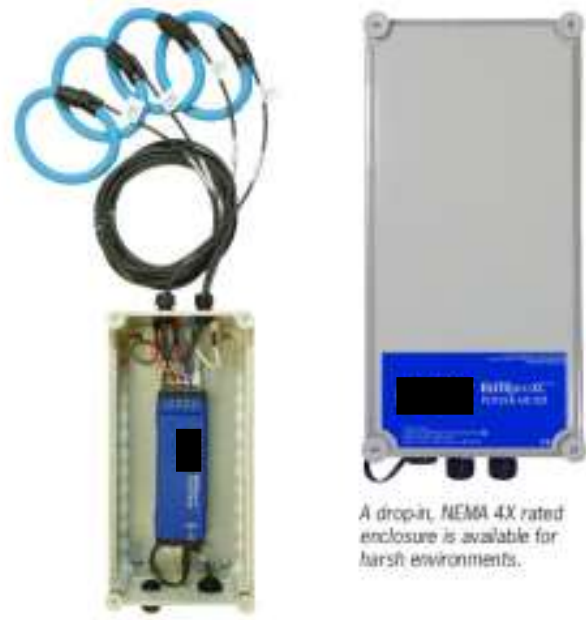

A dropin, fenes $4 x$ rated enclosure is svalatie for harsh ewinonments.

\section{EXCLUSIVE CT PHASE ERROR CORRECTION}

Al CTs exhbit both a ratio error and phase shitt error. The CT phase error results trom the phase relationship of the ingut versus output signal dfference. This inherent phase angle error affects power readings if left uncorrected. When using CTs with a known phase angle error, the ElITEpro XC can correct for this error-making your calculations more accurate, particularly on loads with low power factor.

EFFECT OF CT PHASE ANGLE ERROR ON POWER MEASURENENTS

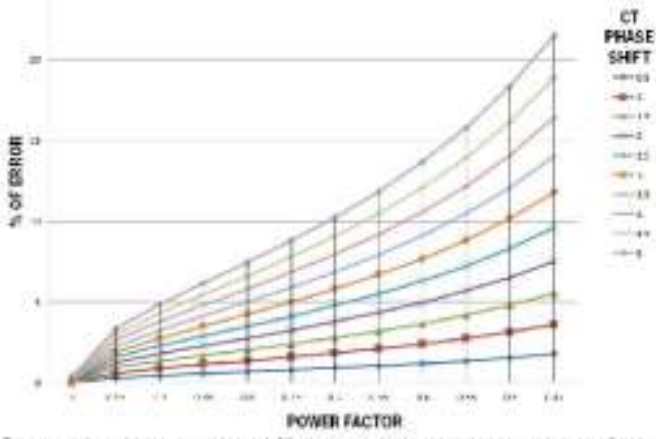

For exampie, with an incorrected 3 phase angle enor and reoorted power fach of 0.5 , there is a correspanding $k W$ eror of $>j 0.4$.

\section{BH-DIRECTIONAL METERING FOR RENEWABLES STUDIES}

The EurtEpro XC design delivers bidirecticnal metering. which is capable of montoring power generated by a renewable energy source versus power imported from the grid-ideal for solar power measurements.

\section{FIELD-PROVEN: RUGGED \& COMPACT}

Measuring in at orly $21.6 \times 6.3 \times 4.7 \mathrm{~cm}\left(8.5^{*} \times 2.5^{*} \times 1.9^{*}\right)$ and we ghing 340 grams (12 ounces), the ELITEpro $x C$ was designed to fi inside panels for safecy and convenience. It nugged plastic housing is even supplied with magnets on the housing to facilate mounting.

Our optional NEMA 4 rated weathertight drop-h enclosure delivers stronger emvironmental integrity, separate voltage plug5, water-tight connectors for both the current leads and the power and communication cables. This option for the ELITEpro XC will meet the stringent requirements of remote data bgging. 


\section{ELITEPRO XC"' START TO FINISH}

\section{EASY SETUP, INSTALLATION, AND DATA RETRIEVAL}

Using the EUTTEro XC on your next project is as easy as $1,2,3$,

1 INSTALL ELOG AND SEND A SETUP
TABLE TO THE ELITEPRO XC

The EUTEpro XC is configured using ELOG sottware. A Setup Table is the fle that programs the logger for an upcoming project. Set parameters such as sampling rate and type of service then send the file to the logger. The ELTEpro XC is now ready for deployment.

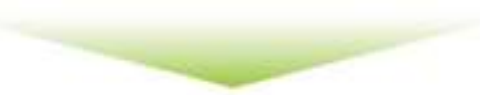

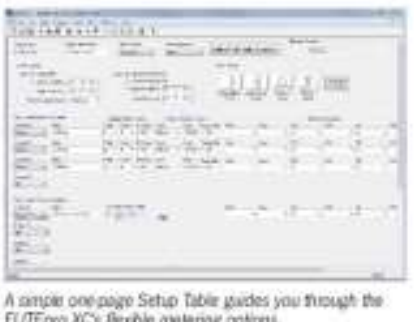

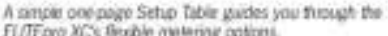

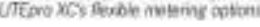

2 CONNECT THE ELITEPRO XC TO THE PANEL YOU NEED TO MEASURE

Installation and connection of the EUTEpro XC is both simole and straightforward. Magnets on the housing facilitate mounting inside electrical cabinets. A variety of internally shunted, snap-on CTs and clip-on voltage leads connect to almost any panel load without turning the power off.

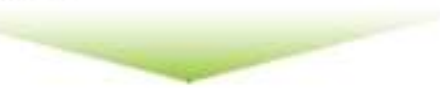

\section{DOWNLOAD THE DATA FROM THE} ELITEPRO XC

At the end of the project, simply comect the ELITEpro XC to a computer with ELOG and downioad the data file. Analyze the data and create graphs in ELOG or export the data as a .c5v file to popular spreadsheet programs, such as Microsolt Exceed.

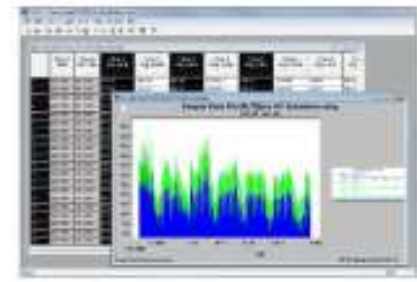

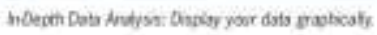

\section{ELOG“ SIMPLE DATA ANALYSIS}

\section{POWERFUL SOFTWARE}

The Windows based ELOG software package is used to program the meter, display metered values, and retrieve and analyze the collected data. ELOG graphisally displays recorded data, performs analysis, and facistates automatic remote data colection. Data is also easily exported to popular spreadsheet and database programs for additional analysis. You are provided with an unamited site ficense to the software with purchase.
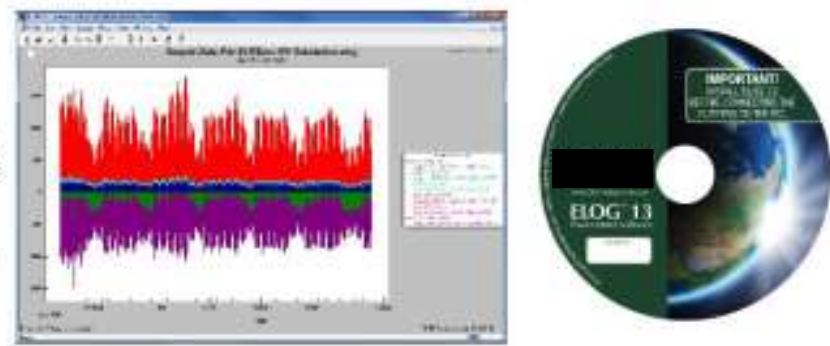


\section{COMMUNICATION OPTIONS}

\section{CONNECT TO THE ELITEPRO XC FOUR WAYS}

\section{STANDARD USB AND ETHERNET CONNECTIONS}

Every ELTEpro XC includes a USB and Ethernet (RJ45) port. Connect directly to the EUTEpro XC via the included Ato B USB cable. Or, have the ELITEpro XC on your local area network for convenient remote data downkad. Supports 10/100 MB Ethemet over Cat 5 or better and can be configured for DHCP or static IP. Schedule automatic data downloads from the logger using AutoPoll sottware fincluded with ELOG). Reconfigure the E.UIEpro XC for a new project without leaving your workstation.

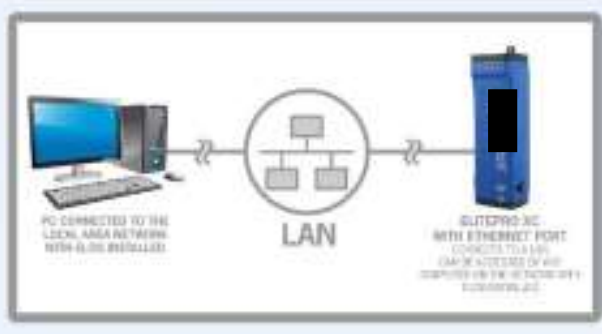

\section{OPTIONAL BLUETOOTH' WIRELESS \\ TECHNOLOGY}

Save time and meet safety requirements by communicating with the EUTEpro XC without removing the electrical panel door. In addition to the standard USB and Ethemet ports, E.JTEpro XC instruments may be equipped with an optional Eluetoottr" interface, which wil allow a PC with a Bluetoothe adapter running ELOG to conect to tho ELITEpro XC over short distances (10-100 feet typical) to send setup tables or download data. PC must support Serial Port Profile (SPP).

\section{OPTIONAL WIFI INTERFACE}

Need a meter on your local area network but do not have a network connection near by? The ELTTEpro XC's optional WiFi interface allows the meter to be connected to a Woffi network and accessed from any PC $\mathrm{cn}$ the network using ELOG software. Or, configure the EUTEpro $\mathrm{XC}$ as its own Wifi hotspot and vew real-time data using a WiFi enabled PC, tablet, or smart phone via a web browser from up to 300 ft. away. Two antema options available: internal anterna $(75-300 \mathrm{ft}$.) or external 3 dbi antenia (150-300 ft). Use ELOG to 5 witch between WiFi and Access Point mode.
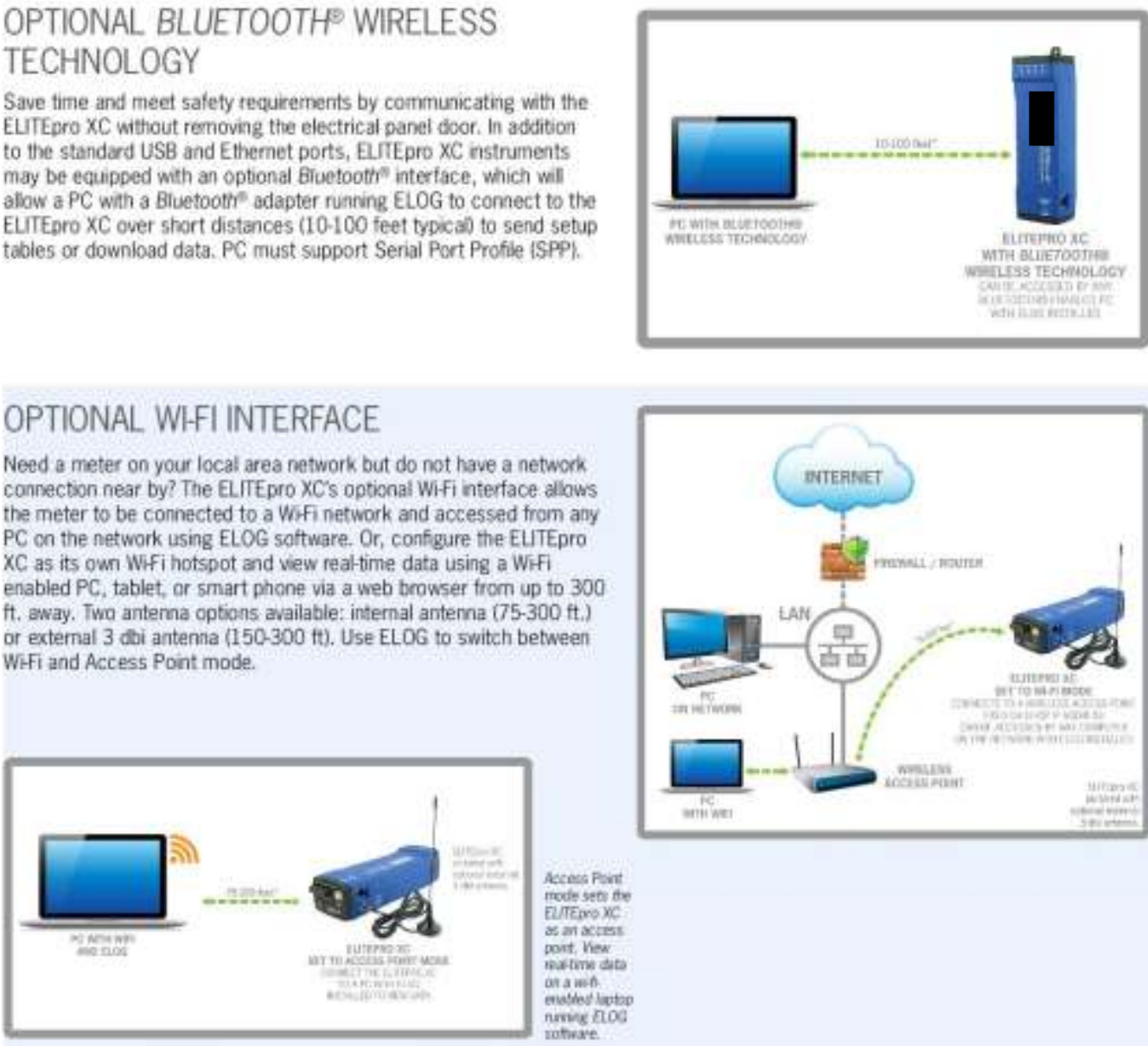
Access Pint
incole sen ine

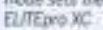
a a a axiss poit, Kex nathe des as a ind ewothet havep nive nod
where. 


\section{ELITEPRO XC" TRANSFORMERS}

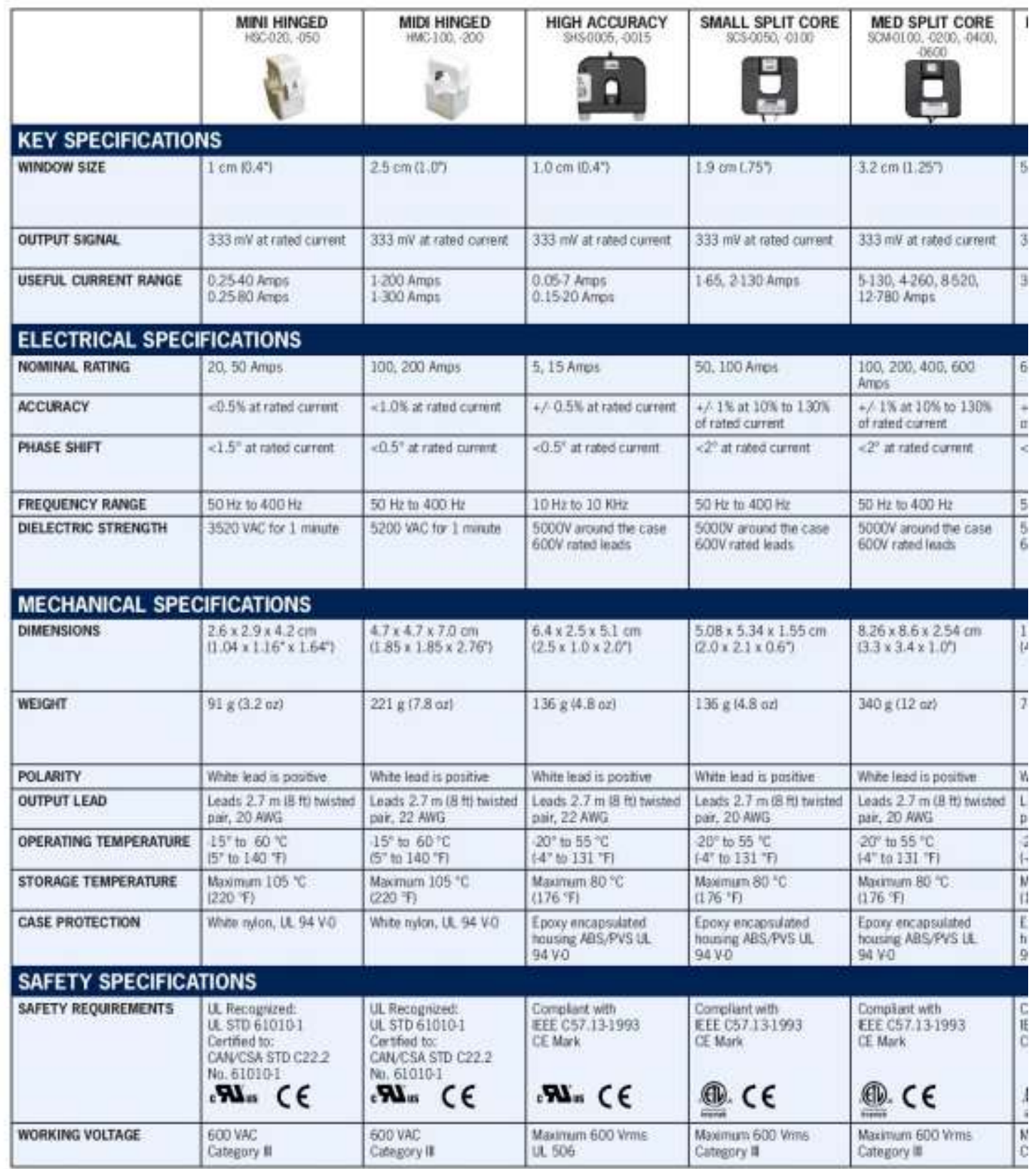


The EUIFpro XC can be equpped wth a wide selection of current transformers, which can be rtercharged deperiting on your proiect reeds. Choose from comoact and economical Split-Coce CTs, converiert Camp-On style CTs, or the versatila Regawsho Flex CFs. AM CT5 are shunted and each type oflers its own particular advartages depending on your appication.

\begin{tabular}{|c|c|c|c|c|}
\hline & $\begin{array}{l}\text { LARGE SPLIT CORE } \\
\text { SOC-0600 }=000\end{array}$ & $\begin{array}{l}\text { 150A CLAMP-ON } \\
\text { CONOLSOEZ }\end{array}$ & 1000A CLAMP-ON & 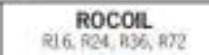 \\
\hline & $5.1 \mathrm{~cm} \cdot 2.07$ & $5.2 \mathrm{~cm} 01.07$ & $5.2 \mathrm{~cm} 12.001$ & $\begin{array}{l}16^{\circ}: 13 \mathrm{~cm}\left(5^{\circ}\right) \\
24^{\circ} 19 \mathrm{~cm}(7) \\
36: 26 \mathrm{~cm}(10) \\
72^{\circ}: 56 \mathrm{~cm}(22)\end{array}$ \\
\hline & $333 \mathrm{~mW}$ at rated current & $\begin{array}{l}333 \mathrm{mv} \text {. } \\
150 \text { Amps } \mathrm{NC}\end{array}$ & $\begin{array}{l}333 \mathrm{~mW} / \mathrm{A} \text {. } \\
1000 \mathrm{ArCS} \mathrm{AC}\end{array}$ & 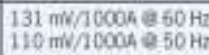 \\
\hline & $30780,20-1300 \mathrm{Amps}$ & 5 to 300 Amps & 20101200 Amps & $\begin{array}{l}\text { EXCJESP,PS3,PS24: } \\
5.5000 \text { Annos: } \\
\text { PS18: } 503500 \text { Amps: }\end{array}$ \\
\hline & 600,1000 kvins & 150 Amps & 1000 Arips & 3000 Amtis \\
\hline & 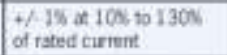 & $\angle 1 \%$ at rated curnent & $+x<18$ & +f 1S roadng \\
\hline & $<2^{2}$ at rated camert & 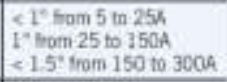 & $+/<<1^{n}$ & $<\mathrm{I}^{\circ}$ at $50 / 50 \mathrm{~Hz}$ \\
\hline & $50 \mathrm{~Hz}$ to $40 \mathrm{CO} \mathrm{Hz}$ & $50 \mathrm{He}$ to $400 \mathrm{~Hz}$ & $30 \mathrm{~Hz}$ 10 $5 \mathrm{kHz}$ & $40 \mathrm{~Hz}$ ta $5000 \mathrm{~Hz}$ \\
\hline & $\begin{array}{l}5000 \mathrm{~V} \text { around the case } \\
600 \mathrm{~V} \text { rated leads }\end{array}$ & 5200 vac, CAT III & $\begin{array}{l}5200 \text { vaC, } 50 / 50 \\
\text { ite between prinary, } \\
\text { secordary and the cuter } \\
\text { case of the hande }\end{array}$ & $\begin{array}{l}7400 \mathrm{VAC} \text { around coil } \\
1000 \mathrm{VmC} \text { rated feads }\end{array}$ \\
\hline & $\begin{array}{l}12.07 \times 12.70 \times 3.06 \mathrm{~cm} \\
(4.8 \times 5.0 \times 1.27\end{array}$ & $\begin{array}{l}8.7 \times 5.0 \times 1.9 \mathrm{~cm} \\
13.4 \times 1.9 \times 0.89\end{array}$ & $\begin{array}{l}21.6 \times 11.1 \times 4.5 \mathrm{~cm} \\
18.5 \times 4.4 \times 1.83\end{array}$ & 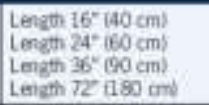 \\
\hline & $748 g(260 x)$ & $136 \mathrm{~g}, 14.8 \mathrm{mi} \mid$ & 500 \& $(5902)$ & 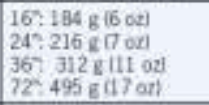 \\
\hline & White leod is postive & Red leat is positive & Fed lead is positive & Wiite lead is pasieve \\
\hline 1 & $\begin{array}{l}\text { Leads } 2.7 \mathrm{~m} \text { is fir wisted } \\
\text { atir, } 20 \text { AWCa }\end{array}$ & $\begin{array}{l}2.4 \mathrm{~m} \mathrm{Bi}) \\
22 \text { goge, } 600 \mathrm{~V}\end{array}$ & $\begin{array}{l}3 \mathrm{~m}\left[118^{\circ}\right) \\
\text { Double insulated }\end{array}$ & $2 \mathrm{~m}(79)$ 'shinided cakin \\
\hline & $\begin{array}{l}20^{\circ} \text { to } 55^{\circ} \mathrm{C} \\
\left(-4^{\circ} \text { b } 131^{7} \mathrm{~F} \mid\right.\end{array}$ & $\begin{array}{l}20^{\circ} \text { to } 70^{\circ} \mathrm{C} \\
\left.\mathrm{H}^{2} \text { to } 158^{\circ} \mathrm{f}\right)\end{array}$ & $\begin{array}{l}10^{\circ} \text { to } 50 \mathrm{c} \\
\left.114^{\circ} \text { to } 120^{\circ} \mathrm{A}\right)\end{array}$ & $\begin{array}{l}10^{2} \text { to }+80^{\circ} \mathrm{C} \\
\left.1+14^{-} \text {to }+175 \mathrm{f}\right)\end{array}$ \\
\hline & $\begin{array}{l}\text { Maimum } 80^{\circ} \mathrm{C} \\
(176 \%)\end{array}$ & $\begin{array}{l}25^{\circ} \text { to } 70 \div 6 \\
113^{\circ} \text { to } 159^{\circ} 71\end{array}$ & $\begin{array}{l}200 \text { to } 70 x \\
\left(4^{2}+10560 \%\right)\end{array}$ & $\begin{array}{l}\text { Macmum } 80^{\circ} \mathrm{C} \\
\qquad 16^{\circ} \mathrm{n}\end{array}$ \\
\hline & 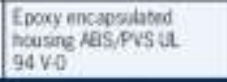 & White AES, UR 94 Vo & $\begin{array}{l}\text { P40UEC 589 } \\
\text { L. } 94 \text { VO }\end{array}$ & $\begin{array}{l}\text { Thermosiastic Ribber } \\
\text { Ui gt vo }\end{array}$ \\
\hline & $\begin{array}{l}\text { Complart whith } \\
\text { EEE C57.13.1993 } \\
\text { CE Mark } \\
\text { (Ai). CE }\end{array}$ & 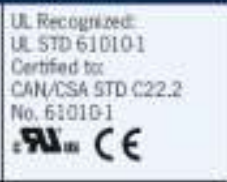 & $\begin{array}{l}\text { CANUCSA STD C22.2 } \\
\text { Na } 610101\end{array}$ & 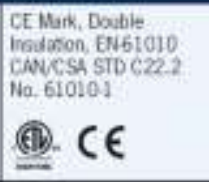 \\
\hline & $\begin{array}{l}\text { Masumim } 600 \mathrm{Vmms} \\
\text { Category } 11\end{array}$ & $\begin{array}{l}\text { Moximus } 600 \text { VMns } \\
\text { Cittegory a }\end{array}$ & $\begin{array}{l}\text { Maxnum } 600 \mathrm{Wms} \\
\text { Cotegary in }\end{array}$ & $\begin{array}{l}\text { Macemam } 1000 \text { Wiras } \\
\text { Catogory }\end{array}$ \\
\hline
\end{tabular}

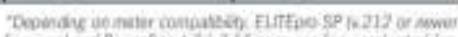

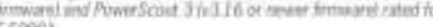
seconat 


\section{ELITEPRO XC"' SPECIFICATIONS}

\begin{tabular}{|c|c|}
\hline SEAVECE TYPE. & 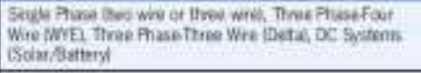 \\
\hline VOUAGE CHWWNELS & 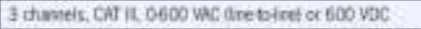 \\
\hline CURREVT CHLAVEELS & 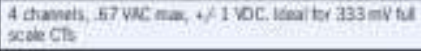 \\
\hline MAXISUM CURRENT ITIPUT & 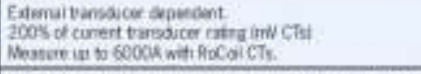 \\
\hline MERSUREMENT TTPE & 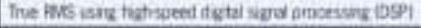 \\
\hline LIVE FPEQLEENCY & DC,90,6016: \\
\hline WWEEFOAM SAMPUNGS & 12 ketr \\
\hline CHNWEL SAMPUNG QRTE & 125 seconds: \\
\hline NTECRATION PERICO & 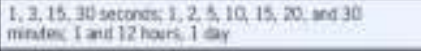 \\
\hline ENDQGY MERSUREMENTS & 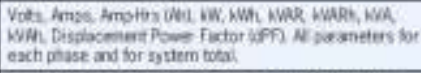 \\
\hline NWALOO MEASUAENEUTS & 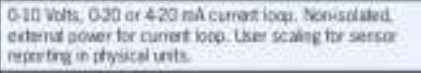 \\
\hline ACCUAACY & 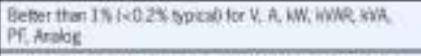 \\
\hline pesocutiow & 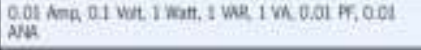 \\
\hline LD EDICATORS & 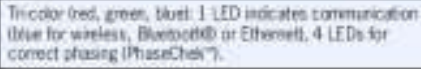 \\
\hline
\end{tabular}

\section{A VARIETY OF VOLTAGE CLIPS}

Several voitage clips options are available to meet your project needs: Croc Clips, Fused Crocs, Colored Leads, or Shark Clips.

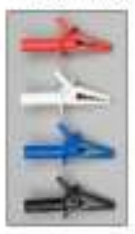

Croc Cint

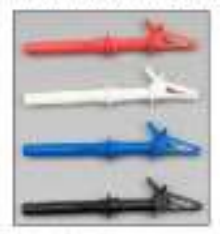

froed choc ave

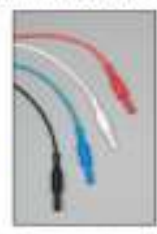

Colsed lests

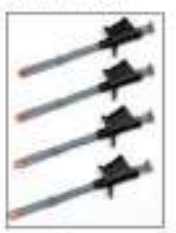

Shas chos

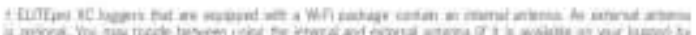

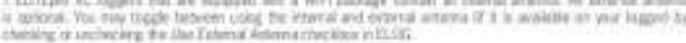
(2)

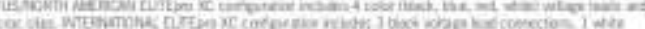

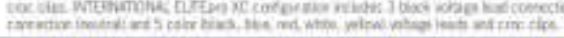

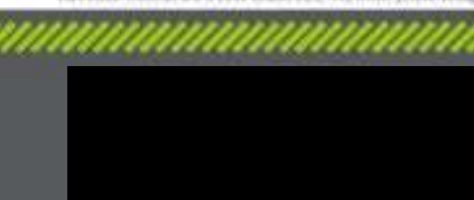

DENT Instruments, Inc.

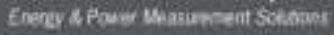

An iso soot:a0os cortined Conpaty ges.5n aquarcine.

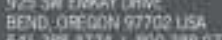

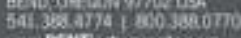

rew DENThtomanticom

\section{POWER}

LWE-POWER

POWER IN

POWER OUT

COMMUNICATIONS

USB (STANDARD)

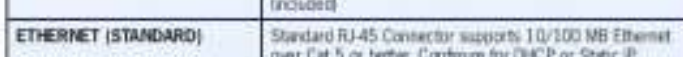

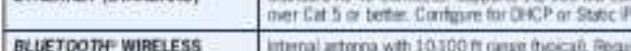

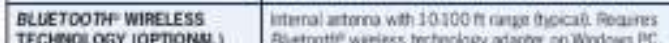

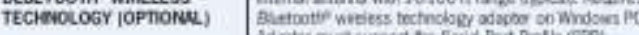

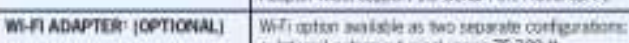

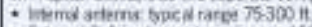

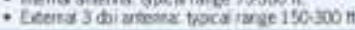

MECHANICAL

OPERATNG TEMPERATURE $\quad 760+60$ "C

\begin{tabular}{|c|c|}
\hline 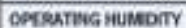 & 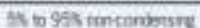 \\
\hline
\end{tabular}

BNCLOSURE TYFE

พยตCH

DIMETISIONS

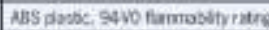

340 g (12: marosi

ORDERING INFORMATION

\section{EXC ORDER STRING}

YOUTAGE LEAD COLORS DNOOK OIC

$\checkmark$ - USTOFTH HEACW.

1 - interentione.

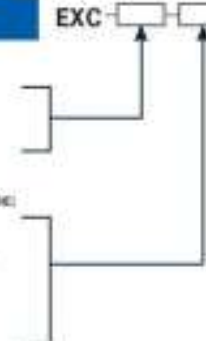

voutace cups ionutinu

c - croctips

F - Rusto dexc clips

s = sharcifs

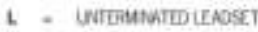

$N$ - rone

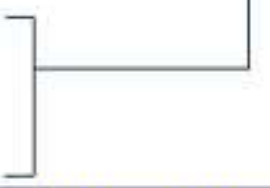

IIIIIIIIIIIIIIIII

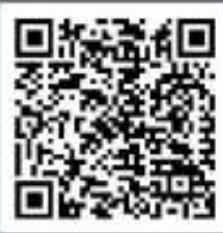

